Portland State University

PDXScholar

Winter 2-7-2013

\title{
3-D Terahertz Synthetic-Aperture Imaging and Spectroscopy
}

Samuel C. Henry

Portland State University

Follow this and additional works at: https://pdxscholar.library.pdx.edu/open_access_etds

Part of the Electromagnetics and Photonics Commons Let us know how access to this document benefits you.

\section{Recommended Citation}

Henry, Samuel C., "3-D Terahertz Synthetic-Aperture Imaging and Spectroscopy" (2013). Dissertations and Theses. Paper 693.

https://doi.org/10.15760/etd.693

This Dissertation is brought to you for free and open access. It has been accepted for inclusion in Dissertations and Theses by an authorized administrator of PDXScholar. Please contact us if we can make this document more accessible: pdxscholar@pdx.edu. 
3-D Terahertz Synthetic-Aperture Imaging and Spectroscopy

by

Samuel C. Henry

A dissertation submitted in partial fulfillment of the requirements for the degree of

Doctor of Philosophy

in

Electrical and Computer Engineering

Dissertation Committee:

Lisa M. Zurk, Chair

Donald Duncan

Branimir Pejcinovic

Martin Siderius

Dean Atkinson

Portland State University

2013 
(c)2013 Samuel C. Henry 


\begin{abstract}
Terahertz $(\mathrm{THz})$ wavelengths have attracted recent interest in multiple disciplines within engineering and science. Situated between the infrared and the microwave region of the electromagnetic spectrum, $\mathrm{THz}$ energy can propagate through non-polar materials such as clothing or packaging layers. Moreover, many chemical compounds, including explosives and many drugs, reveal strong absorption signatures in the $\mathrm{THz}$ range. For these reasons, $\mathrm{THz}$ wavelengths have great potential for non-destructive evaluation and explosive detection.

Three-dimensional (3-D) reflection imaging with considerable depth resolution is also possible using pulsed $\mathrm{THz}$ systems. While THz imaging (especially 3-D) systems typically operate in transmission mode, reflection offers the most practical configuration for standoff detection, especially for objects with high water content (like human tissue) which are opaque at $\mathrm{THz}$ frequencies. In this research, reflection-based $\mathrm{THz}$ synthetic-aperture (SA) imaging is investigated as a potential imaging solution. $\mathrm{THz} \mathrm{SA}$ imaging results presented in this dissertation are unique in that a 2-D planar synthetic array was used to generate a 3-D image without relying on a narrow time-window for depth isolation [1]. Novel THz chemical detection techniques are developed and combined with broadband $\mathrm{THz}$
\end{abstract}


SA capabilities to provide concurrent 3-D spectral imaging. All algorithms are tested with various objects and pressed pellets using a pulsed THz time-domain system in the Northwest Electromagnetics and Acoustics Research Laboratory (NEAR-Lab). 


\section{Acknowledgments}

I want to acknowledge and thank the Office of Naval Research, the National Science Foundation, and the M. J. Murdock Charitable Trust for generous grants that support $\mathrm{THz}$ research at Portland State.

I would also like to thank my advisor, Dr. Lisa Zurk, for supporting all my graduate studies at Portland State University. Dr. Zurk has offered a tremendous amount of guidance for which I am extremely grateful. Dr. Zurk has established a wonderfully collaborative research environment that has been greatly beneficial to myself and many other individuals. I am truly grateful for all the wonderful opportunities while working with her at the NEAR-Lab.

I also deeply appreciate the help of Dr. Donald Duncan and his endless hours of instruction, support and conversation.

Thanks to Drs. Branimir Pejcinovic, Martin Siderius and Dean Atkinson for stimulating discussions and general advice in the advancement of my research work.

I would also like to give thanks Dr. Rick Campbell for all his insight into the 
area of experimental work, and for teaching me to always find ways to challenge myself.

Thanks to Drs. Lisa Zurk, Donald Duncan, Branimir Pejcinovic, Martin Siderius and Dean Atkinson for serving on my Committee.

I am indebted to my coworkers at the NEAR-Lab for their support. In particular, thanks to Gabe Kniffin, Scott Schecklman, Lanfranco Muzi, Elizabeth Küsel, George Ogden, John Gebbie, Reid McCargar, Alex Higgins, Eric Sorensen, and Richard Campbell for countless times helping me and providing advice. None of this work would be possible without all your help. 


\section{Table of Contents}

Abstract

Acknowledgments $\quad$ iii

List of Tables $\quad$ ix

List of Figures $\quad x$

1 Motivation for Dissertation Work 1

1.1 Contributions and significance of this work . . . . . . . . . 2

1.2 Summary of dissertation . . . . . . . . . . . . . . 4

2 Literature Review $\quad 6$

$2.1 \mathrm{THz}$ technology . . . . . . . . . . . . . . . . 7

$2.2 \mathrm{THz}$ imaging and tomography . . . . . . . . . . . . . . 11

2.3 Synthetic-aperture imaging . . . . . . . . . . . . . . . . . . 16

2.4 Spectral imaging and identification . . . . . . . . . . . . 19

3 THz Time-Domain Spectroscopy 21 
3.1 Picometrix T-Ray 4000 pulsed THz system . . . . . . . . . . . . . 27

3.2 Effects of scattering on lactose reflection signature . . . . . . . . 29

4 Robust Chemical Detection Techniques 35

4.1 Matched filter detection . . . . . . . . . . . . . 35

4.2 Correlation processing . . . . . . . . . . . . . 37

4.3 Squared residual detection . . . . . . . . . . . . . . . . 41

4.4 Validation of detection techniques . . . . . . . . . . . . . 42

4.4.1 Imaging results using two-sided pellet . . . . . . . . . . . 43

4.4 .2 Performance metrics . . . . . . . . . . . . . . . . 47

4.5 Monte Carlo Kirchhoff simulations . . . . . . . . . . . . . . 50

4.5.1 Case \#1: lactose (target) versus HDPE . . . . . . . . . . . 52

4.5.2 Case \#2: C-4 versus (target) lactose . . . . . . . . . . . . 54

5 Synthetic-Aperture Processing $\quad 57$

5.1 Theoretical imaging formulation . . . . . . . . . . . 57

5.1.1 Frequency-domain synthetic-aperture processing using a virtual source . . . . . . . . . . . . . . . 60

5.1.2 Broadband 3-D synthetic-aperture processing . . . . . 63

5.2 Additional processing considerations . . . . . . . . . . 64

5.2.1 $\mathrm{THz}$ beam distortion .............. . . 65

5.2.2 Mitigation of system-level etalon effects . . . . . . . . . 68 
5.3 3-D broadband $\mathrm{SA} \mathrm{THz} \mathrm{imaging} \mathrm{results} \mathrm{.} \mathrm{.} \mathrm{.} \mathrm{.} \mathrm{.} \mathrm{.} \mathrm{.} \mathrm{.} \mathrm{.} \mathrm{.} \mathrm{.} \mathrm{.} \mathrm{.} 70$

5.3.1 Surface profiling with metallic interface . . . . . . . . 71

5.3.2 3-D Target depth localization ............ . 74

5.3.3 Volumetric imaging of 3-D dielectric structures . . . . . . . 77

5.3.4 HDPE pellet with multiple embedded scatterers . . . . . 79

5.4 Characterization .................... 82

5.4.1 Broadband resolution improvement . . . . . . . . . 83

5.4 .2 Depth localization ............... 84

5.4.3 Bandwidth comparison ............. 86

6 Synthetic-Aperture Based 3-D Spectral Imaging 88

6.1 Theoretical formulations . . . . . . . . . . . . . . . . 88

6.1.1 Correlation processing . . . . . . . . . . . 89

6.1.2 Frequency differencing ............... . . 90

6.2 Spectral profiling results with two-sided pellet . . . . . . . . 92

6.2.1 Quantitative performance of spectral profiling . . . . . 94

7 3-D THz Spectral Imaging of Dielectric Spheres 98

7.1 Matched-field imaging . . . . . . . . . . . . . . . 98

7.2 Simulations details . . . . . . . . . . . . . . . 100

7.3 3-D Spectral imaging results . . . . . . . . . . . . . 103

7.4 Quantitative performance comparison . . . . . . . . . 105 
8 Conclusion and Future Work

8.1 Conclusion . . . . . . . . . . . . . . . . 107

8.2 Future work . . . . . . . . . . . . . . . . 110

8.3 Contributions to the $\mathrm{THz}$ community . . . . . . . . . . . 111

References

Appendix A Study on Dielectric Tube Waveguides

A.1 Propagation Physics . . . . . . . . . . . . . . 127

A.1.1 Horn Excitation . . . . . . . . . . . . . . . 132

A.2 Measurements . . . . . . . . . . . . . . . . 134

A.3 Comparison of Model and Measurement . . . . . . . . . . 136

A.4 Simulated Results . . . . . . . . . . . . . . . . 138

Appendix B Generation of Receiver Operating Characteristics 141

Appendix C Computationally Efficient Implementation of Synthetic-

Aperture Imaging

Appendix D Characterization of Virtual Source

Appendix E Virtual Source Drift Correction

Appendix F Synthetic-Aperture Refraction Correction for Dielectric Structures 


\section{List of Tables}

3.1 List of sandpaper statistics . . . . . . . . . . . . . . . . . . 30

5.1 Imaging resolution metric comparison between SA depth focusing and time-gate depth focusing. . . . . . . . . . . . . 82

6.1 Comparison of surface statistics using THz profiling method . . . 96 


\section{List of Figures}

2.1 Venn diagram showing three areas feeding into this research work 6

2.2 The "THz Gap" shown within the greater electromagnetic spectrum. 7

2.3 Optical and terahertz image circuit board with Portland State University $\log _{0} \ldots \ldots \ldots \ldots \ldots \ldots$

2.4 Example of terahertz waveform and associated frequency spectrum. 14

3.1 Geometry for transmission mode spectroscopy . . . . . . . . . . 21

3.2 Lorentz material properties for lactose . . . . . . . . . . 25

3.3 Kirchhoff realizations showing scattered field and negative derivative 26

3.4 Picmometrix T-Ray 4000 pulsed terahertz system $\ldots \ldots \ldots$. . 28

3.5 T-Ray 4000 arranged in bistatic configuration . . . . . . . . . 29

3.6 Photos of three rough lactose samples . . . . . . . . . . . . 30

3.7 Reflection spectra from rough lactose samples . . . . . . . . . . 31

3.8 Diagram of diffuse scattering geometry $\ldots \ldots \ldots$

3.9 Diffuse reflection spectra from rough lactose samples . . . . . . 34

4.1 Phase of the lactose reflection coefficient $\ldots \ldots \ldots$ 
4.2 Illustration of correlation processing . . . . . . . . . . . . . . . 40

4.3 Illustration of the squared residual method . . . . . . . . . . . . . 42

4.4 Photo and imaging configuration for two-sided pellet made with lactose and high-density polyethylene . . . . . . . . . . . . 43

4.5 $\mathrm{THz}$ imaging results using two-sided pellet . . . . . . . . . . . . 44

4.6 Plot of correlation processing output versus squared residuals . . . 46

4.7 Receiver operating characteristics for two-sided pellet . . . . . . . 49

4.8 Receiver operating characteristics for Monte Carlo simulation . . . 53

4.9 Amplitude and phase of the C-4 reflection coefficient at normal incidence . . . . . . . . . . . . . . . . 55

4.10 Receiver operating characteristics for Monte Carlo simulation . . . 56

5.1 One-dimensional linear array being used in a synthetic-aperture

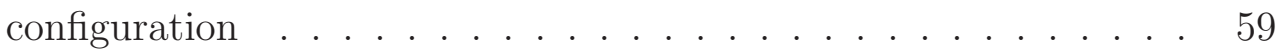

5.2 2-D planar array used in a synthetic-aperture configuration . . . . 61

5.3 Demonstration of distortion due to irregular beampatterns . . . . 66

5.4 Demonstration of background subtraction . . . . . . . . . 69

5.5 Surface proflie of American Dime . . . . . . . . . . . . . . 72

5.6 Surface profile of American Dime . . . . . . . . . . . . . 73

5.7 High-density polyethylene sample with embedded ball bearings . . 74

5.8 Synthetic-aperture images of sample with embedded ball bearings 76 
5.9 Synthetic-aperture images of floppy drive power connector . . . . 77

5.103 -D image of floppy drive power connector . . . . . . . . . . 78

5.11 Comparison between $\mathrm{THz} 3-\mathrm{D}$ imaging methods . . . . . . . . . . 80

5.12 Synthetic-aperture and time-gated 3-D images of sample with multiple ball bearings . . . . . . . . . . . . . . . . 81

5.13 Depth of focus for two broadband averages . . . . . . . . . . . 83

5.14 Depth localization demonstration . . . . . . . . . . 85

5.15 Depth resolution is plotted as a function of bandwidth . . . . . . 86

6.1 Reflected amplitude from lactose, with two highlighted regions used for frequency differencing . . . . . . . . . . . . . . 91

6.2 The two-sided pellet synthetic-aperture configuration . . . . . . . 92

6.3 Terahertz spectral profiling results . . . . . . . . . . . . . . . . 93

6.4 Receiver operating characteristics for spectral profling . . . . . . . 95

7.1 Monte Carlo configuration showing the locations of distributed dielectric spheres comprised of lactose or high-density polyethylene 101

7.2 Geometry of a plane wave impinging on a sphere . . . . . . . . . . 102

7.3 Location and 3-D SA image of dielectric spheres . . . . . . . . . . 104

7.4 3-D THz images of dielectric spheres comprised of high-density polyethylene and lactose . . . . . . . . . . . . . . 105

7.5 Receiver operating characteristics for dielectric sphere images . . . 106 


\section{Chapter 1}

\section{Motivation for Dissertation Work}

This work addresses the field of 3-D terahertz $(\mathrm{THz})$ imaging for a wide range of applications such as explosives detection and non-destructive evaluation. In the past 20 years, THz sources and receivers have significantly increased in efficiency while becoming more compact and affordable. Consequently, different types of imaging systems are currently being investigated by a variety of scientists and engineers. The approach demonstrated in this work is among the first of its kind, combining multifaceted tools from synthetic-aperture radar and spectral imaging. The end goal is to provide innovative solutions for $\mathrm{THz} 3-\mathrm{D}$ spectral imaging. The motivation for this research is:

1. THz source bandwidths are now large enough to view spectral fingerprints for a variety of chemicals, such as drugs and explosives. To fully utilize this portion of the spectrum for security and non-destructive evaluation applications, imaging systems must be able to concurrently provide 3-D imaging and material characterization.

2. Current $\mathrm{THz}$ systems being researched have trade-offs between spectroscopic identification performance and depth resolution in 3-D imaging. By 
using advanced synthetic-aperture (SA) image processing in conjunction with spectral detection techniques, these issues can be mitigated.

3. Many recent $\mathrm{THz}$ tomographic imaging configurations require a rotational platform and often operate in transmission mode, which may be difficult to implement in an integrated system. A 2-D synthetic array that utilizes backscattered radiation can produce 3-D THz images with fine depth resolution; and a planar geometry will likely be better suited for a manufacturable THz 3-D imaging system.

4. Often, in a real-world imaging situation, a target material is comprised of inhomogeneous scatterers and rough surfaces, resulting in random fluctuations in recorded spectra. This effect can cause significant shortcomings in conventional spectroscopy methods, and in general are not mitigated with increased signal strength. Advanced signal processing techniques are required for robust detection of resonance features in real-world conditions.

\subsection{Contributions and significance of this work}

The following steps, completed as part of the final dissertation, have expanded the body of knowledge in the $\mathrm{THz}$ imaging community.

1. Developed robust $\mathrm{THz}$ resonance feature detection methods that are wellsuited for reflection mode. Correlation processing and squared residual 
methods both utilize sharp magnitude and phase features that are present in scattered spectra from explosives or other chemical compounds. Each method's detection performance is robust to scattering effects due to rough surfaces.

2. Implemented a broadband 3-D THz SA imaging algorithm. This implementation was the first of its kind, utilizing a 2-D array and to create high resolution 3-D THz images. A broadband $\mathrm{THz}$ system will provide 3-D imaging with significant depth and lateral resolution. This algorithm was based on a modified edition of conventional synthetic-aperture radar techniques.

3. Developed 3-D SA spectral imaging algorithms by integrating robust feature detection techniques with a multiposition sensor array. The 3-D spectral imaging techniques that were developed were correlation processing, frequency differencing and matched-field imaging.

4. Applied advanced image processing algorithms to real data measured in the NEAR-Lab THz measurement facility. Once the data were measured, a sequence of processing steps were developed to create the final results. A variety of methods were investigated and quantitatively compared in an effort to provide the best possible $\mathrm{THz}$ imaging solutions. 


\subsection{Summary of dissertation}

The results shown throughout this work are primarily based on data measured with a Picometrix T-Ray $4000 \mathrm{THz}$ system in the Northwest Electromagnetic and Acoustics Research Laboratory (NEAR-Lab). The pulsed system records time-domain waveforms with accurate phase information necessary for syntheticaperture phased-array processing.

Each of the developments presented in this dissertation have been well received in the $\mathrm{THz}$ imaging community. This section outlines the body of the dissertation, and provides a brief summary of each chapter for a quick reference guide.

Chapter 2 provides a literature search and introduction to $\mathrm{THz}$ technology, $\mathrm{THz}$ imaging and the vast implications of this portion of the electromagnetic spectrum. The literature search also incorporates synthetic-aperture radar and remote sensing theory.

Chapter 3 first provides details on current THz material detection techniques. The chapter also summarizes a study on scattering effects of derivative spectra at specular and diffuse angles from an explosive simulant.

Chapter 4 shows formulations for three robust chemical detection techniques (two of which are contributions to this dissertation), namely matched filtering, correlation processing, and squared residual processing. Later in this chapter, these techniques are validated with images of a two-sided pellet constructed of 
$\alpha$-lactose and high-density polyethylene (HDPE).

Chapter 5 describes broadband $\mathrm{THz} 3-\mathrm{D}$ SA imaging techniques that have been developed as part of this dissertation, and shows imaging results from various dielectric and metallic objects in addition to characterization of imaging performance.

Chapter 6 combines broadband 3-D THz imaging techniques described in Chapter 5 with material detection techniques such as correlation processing and frequency differencing. Spectral surface profiles of a two-sided pellet constructed of $\alpha$-lactose and HDPE are presented and discussed.

Chapter 7 introduces the matched-field imaging technique, an extension of the matched filter for 3-D spectral imaging. This chapter provides 3-D spectral imaging results from a Monte Carlo simulation comprised of two different species of dielectric spheres.

Chapter 8 gives concluding remarks and potential future research topics in the area of $\mathrm{THz} 3-\mathrm{D}$ imaging for both chemical detection and non-destructive evaluation. Also provided in this section is list of conference and journal publications that were published as part of this research. 


\section{Chapter 2}

\section{Literature Review}

There are three main research fields that all contribute to the 3-D THz spectral imaging research presented in this dissertation. Those areas are $\mathrm{THz}$ technology, synthetic-aperture radar (and imaging), and spectral imaging. Within each of these individual areas, there exists a large body of work with many publications. Synthetic-aperture imaging and spectral imaging are each very mature fields while $\mathrm{THz}$ imaging is relatively new.

The aim of this work is to combine all three of these areas for simultaneous imaging and spectral characterization, as shown in the Venn diagram in Figure 2.1. To the best knowledge of the author, at the current time, there has been no published work that combines each of these three areas. While this work

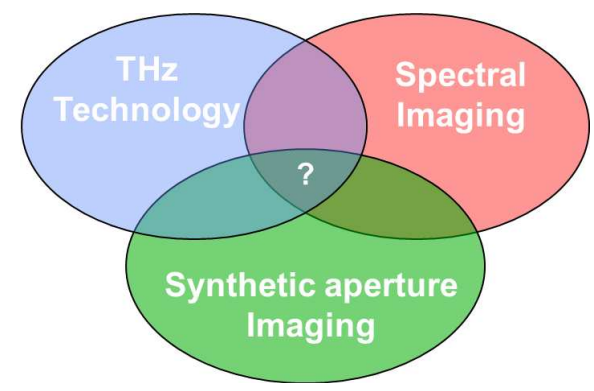

Figure 2.1: Venn diagram showing three areas feeding into this research work: $\mathrm{THz}$ technology (and imaging), synthetic-aperture imaging, and spectral imaging. 
focuses on explosive (or explosive simulant) detection, the methods discussed are widely applicable.

\subsection{THz technology}

Terahertz imaging has attracted recent interest from multiple disciplines within engineering and science. The $\mathrm{THz}$ region of the electromagnetic (EM) spectrum, shown in Figure 2.2, covers $0.3 \times 10^{12}$ to $3 \times 10^{12} \mathrm{~Hz}$, between the infrared and microwave regions. With the improvement of ultra-fast laser technology, $\mathrm{THz}$ sources and detectors have been increasing in efficiency since the 1990s [2]. Unsurprisingly, THz time-domain systems have entered a period of significant innovation as researchers continue to find new applications. In particular, $\mathrm{THz}$ imaging technology holds promising applications in counter-terrorism efforts and non-destructive evaluation.

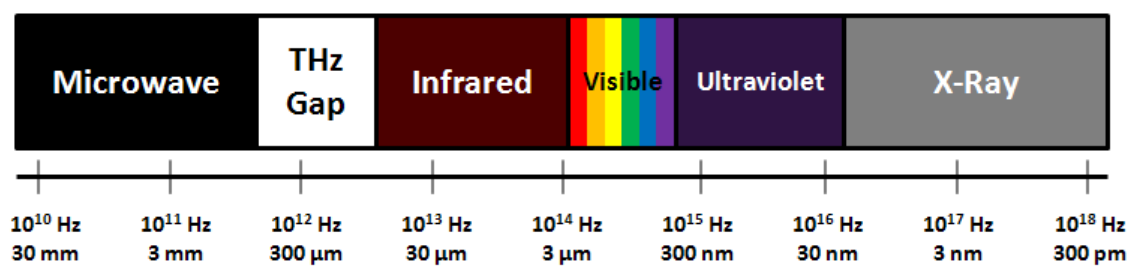

Figure 2.2: The "THz Gap" shown within the greater electromagnetic spectrum.

THz wavelengths, ranging from $100 \mu \mathrm{m}$ to $1 \mathrm{~mm}$, are much shorter than wavelengths in the microwave region, providing higher resolution imaging capability than microwave sensors. Another attractive feature of the $\mathrm{THz}$ region is that 
many non-polar molecules, such as clothing and packaging materials, become transparent at $\mathrm{THz}$ wavelengths, while metallic objects contribute strong reflections. Figure 2.3 shows a THz image (right) of an etched copper circuit board with Portland State University's current logo, imaged with a Picometrix T-Ray 4000 pulsed THz system with a one-inch focusing lens. Also shown is an optical photo of the circuit board (left). The copper remaining on the board after etching provides the brighest returns in the image.

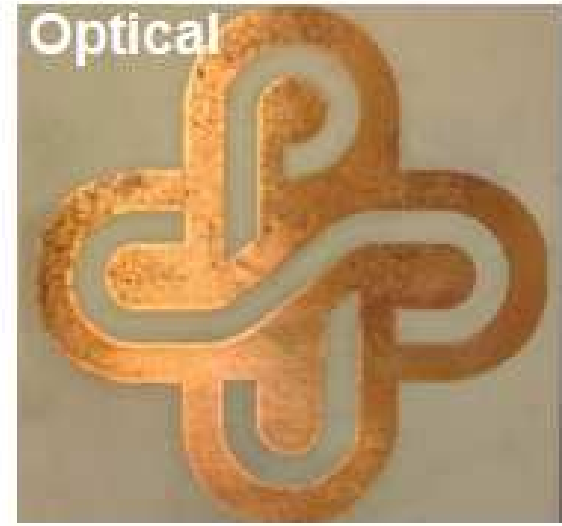

$-1 \mathrm{~cm}$

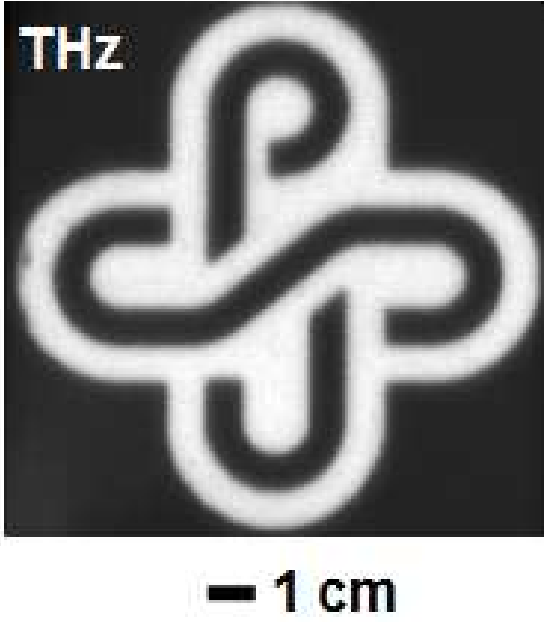

Figure 2.3: (left) Optical photo of etched copper circuit board with Portland State University logo. (right) THz image of the same circuit board.

While many materials are transparent in the $\mathrm{THz}$ range, many explosive materials and other chemical compounds, contain unique spectral signatures. This makes it well-suited for remote sensing security applications. Currently, airports across the world employ millimeter-wave scanners in addition to other security 
standbys such as x-rays and physical searches. These scanners, generally operating at less than $100 \mathrm{GHz}$, produce non-ionizing radiation that is safe for human exposure, and produce images showing what a person may be carrying underneath clothing layers. While these systems are capable of spotting hidden physical objects, they provide little information about the material of interest. A growing concern is the possibility of improvised explosive devices (IED) passing security screenings at airports or military checkpoints. Advanced systems must be able to concurrently image and identify illicit materials by pushing higher into the $\mathrm{THz}$ range and fully harnessing the potential of these unique wavelengths.

There are many research-based ideas for $\mathrm{THz}$ sensors that have been demonstrated for future use. However, there are several commercial $\mathrm{THz}$ applications that are already in use. Radar systems built using the lower $\mathrm{THz}$ range have already found their way into modern "smart cars" to sense other cars or objects in close proximity using $77 \mathrm{GHz}$ transceivers [3]. In communications, 60 $\mathrm{GHz}$ base stations in close range line-of-site communications are rapidly being developed [4]. For several years, millimeter-waves have been used in passive atmospheric sensing in astronomy [5]. There will likely be many other commercial and scientific applications if $\mathrm{THz}$ sources continue to increase in efficiency and become more compact and affordable.

While $\mathrm{THz}$ systems show promise, a significant amount of work remains. 
Propagation effects, such as narrow water vapor absorption lines, are often seen in recorded waveforms and can alter $\mathrm{THz}$ spectra [6]. For this reason, a reference waveform is usually recorded before the sample object to account for propagation effects and the transmitted spectrum of the THz pulse, although they cannot be completely removed. $\mathrm{THz}$ systems are still largely signal-to-noise ratio (SNR) and range limited, and higher efficiencies are needed for practical imaging environments. Indeed, there is continuing progress in extending the efficiency and range of THz systems [7].

Scattering effects are another significant issue in the $\mathrm{THz}$ range [8]. In the microwave region, many common surfaces, such as concrete walls, appear as smooth, largely specular reflectors. At the same time, these surfaces are rough at optical wavelengths, greatly exceeding the Raleigh criterion for surface roughness. In the $\mathrm{THz}$ range, however, many of these surfaces appear somewhere in between rough and smooth [9]. While surface roughness can add complexity in a detection algorithm, it also produces diffuse returns which allow for multiple viewing angles $[10,11]$. In addition, etalon effects (multiple reflections) are often seen at $\mathrm{THz}$ frequencies and can considerably alter $\mathrm{THz}$ spectra [12]. Yet these scattering effects remain poorly understood. Fully understanding the physics of these effects and their implications removes a significant barrier in $\mathrm{THz}$ technology. 


\section{$2.2 \quad \mathrm{THz}$ imaging and tomography}

The microwave region of the EM spectrum has developed into a robust imaging field. Microwave wavelengths have been shown to be quite capable of producing images of larger objects, with applications such as terrestrial mapping in both active and passive configurations [13]. The microwave region has also been proven useful in low resolution medical imaging applications such as breast tumor detection and imaging of other tissues [14], [15]. Microwave wavelengths can penetrate a variety of materials; however, they are not short enough to generate high-resolution images for package screening. However, THz wavelengths, ranging as low as $100 \mu \mathrm{m}$, are short enough for such security applications. THz sensors can provide a higher resolution imaging capability in this area while also providing spectroscopic information about the imaged material.

THz imaging has been studied extensively to detect explosive devices [16]. There are, in fact, multiple explosive detection techniques such as infrared tunable laser spectroscopy [17], Raman spectroscopy [18], and trace detection using

chemical sensors [19]. However, each of these techniques is an invasive technique. For example, explosive trace detection requires a swab of material to be collected by swiping across someone's person. Meanwhile, infrared laser spectroscopy detection requires a pure explosive sample that more closely resembles a laboratory prepared sample than real-life explosive devices. THz wavelengths, on the other 
hand, can detect explosives efficiently, while offering several other advantages such as penetration through clothing $[16,20]$.

Typically, THz explosive detection (and infrared techniques) use a transmission configuration. The material is typically identified by selecting narrow frequency bands around absorption peaks. In reflection mode, the situation is more complicated, as there are no longer distinct peaks. Instead, reflection signatures generally resemble an offset wavelet function. One method to resolve a target signature in reflection mode is to take the first-order derivative of the reflected amplitude with respect to frequency $[10,21]$. While the reflected phase also contains target signatures, it is difficult to extract information from the phase directly due to unwrapping errors. Instead, the second-order derivative of the phase with respect to frequency can be used [21]. Each of these techniques allows one to view the reflection spectrum as if it were absorption, and identify the material based on the location and strength of the peaks. However, these techniques involve first and second-order derivatives, which amplify the noise, particularly at higher frequencies with lower SNR.

In addition to explosives detection, there are many other possible applications for $\mathrm{THz}$ waves. Just like neighboring wavelengths in the infrared and microwave regions, there are a host of non-destructive evaluation and remote sensing applications that have been developed as the $\mathrm{THz}$ gap has become more accessible. 
For example, researchers have recently shown $\mathrm{THz}$ to be effective in detecting faults in integrated circuits [22]. Typically, failure analysis of an integrated circuit requires removal of the packaging structure, or "decapping." THz sensors are able to penetrate packaging layers to reveal interconnecting failures while leaving packaging intact. $\mathrm{THz}$ sensors have also been used in a similar configuration to detect cracks in manufactured solar panels [23].

$\mathrm{THz}$ reflection imaging can be used for a wide range of applications within non-destructive evaluation. For example, THz sensors have been used to preserve information from historical documents or artifacts. Recent THz work includes inspecting layers of artwork, reading text from ink inside pages of historic and possibly fragile manuscripts, and investigating corroded and mineralized copper alloys on historical artifacts [24-27]. Many of these sensing capabilities are useful as a single measurement, but all can be enhanced through combination with an imaging configuration.

THz wavelengths are also very sensitive to liquid water. For this reason, THz sensors are capable of measuring water content in a sample with a reflectionmode THz time-domain system [28]. Recently, THz systems have demonstrated the ability to sense hydration levels in plants and human skin [29]. THz systems can monitor water content in paint as it dries on the cage of an automobile [30]. In addition, $\mathrm{THz}$ systems have great potential in biomedical applications. It 
has been shown that $\mathrm{THz}$ sensors can detect excised breast tumors and identify resonances in biological structures such as DNA [31,32].

While there are many ways to produce $\mathrm{THz}$ radiation, only two methods will be discussed here. One method involves heterodyning electronic microwave oscillators with devices such as GaAs Schottky barrier diodes [33]. THz waves can also be generated by mixing down from the infrared or optical region [34-38]. Both techniques require some form of non-linear behavior, whether it's a circuit component such as a diode (electronic-based source) or a non-linear crystal (laserbased source).

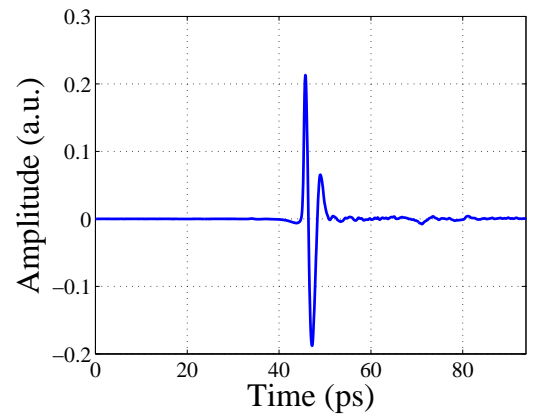

(a)

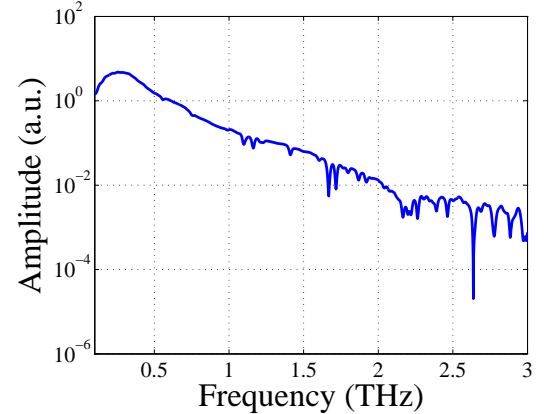

(b)

Figure 2.4: (a) A THz pulse measured with the Picometrix T-Ray 4000 at the NEAR-Lab. (b) Spectrum of THz pulse is obtained via a Fourier transform, as computed by (3.1).

THz sources are typically either continuous wave, meaning that they operate at a single frequency (narrowband), or pulsed (broadband). This work uses the later, a broadband source that generates $\mathrm{THz}$ waveforms by rectifying very short optical pulses ( 100 femtoseconds in duration). This is done by illuminating 
a biased, photo-conductive substrate, which acts as an antenna to create timedomain $\mathrm{THz}$ pulses that are reconstructed with electro-optic sampling [39]. A typical THz pulse and associated frequency spectrum is shown in Figure 2.4. The time-domain nature of these sources allows easy access to depth information from a material in a similar fashion to a time-domain reflectometer.

All of these systems are easily adapted to an imaging configuration by mechanically changing the position or orientation of the source to create a twodimensional grid of pixels $[40,41]$. Each pixel records either a time-domain waveform or swept frequency scattering parameters, depending on the $\mathrm{THz}$ source. 3-D THz imaging and tomography have received a lot of recent interest, as high resolution images can be produced through transparent materials [42]. Typically, in 3-D imaging or tomography, depth information can either be acquired with a true time-domain system, or with swept frequency radar chirp processing to provide range resolution [43]. THz time-domain reflection imaging, in particular, has been a widely successful way to view hidden layers in dielectric materials [44]. In addition, 3-D imaging can simultaneously offer broadband information about the reflection spectrum of the imaged material. 3-D THz imaging systems that provide concurrent spectral information have been researched for applications in explosives detection, the pharmaceutical industry, and other areas [45-48]. 
One such 3-D THz imaging system used for 3-D chemical mapping uses a narrow collimated beam that illuminates a 3-D volume and utilizes the time-domain nature of the THz pulse to get depth information [1], much like B-scan pulsedecho ultrasound. The THz collimated beam is raster-scanned in two dimensions. Slices in depth are produced by isolating sections of the time-domain return, and the depth resolution is thus related to the width of the time window. However, spectroscopic information is produced by transforming from the time domain to the frequency domain. And because the width of the time window is inversely proportional to frequency resolution, 3-D imaging using this time-gating method inherently limits spectroscopic capability.

Another 3-D imaging technique relies on fixed-focus confocal imaging. The $\mathrm{THz}$ beam is focused onto a small focal volume, which is raster-scanned in three dimensions. This can achieve sub-millimeter depth resolution, depending on the numerical aperture of the lens. However, the 3-D image takes a long time to create as mechanical scanning is required to form the depth dimension.

\subsection{Synthetic-aperture imaging}

Synthetic-aperture radar is a remote sensing modality capable of imaging large areas at high resolution. Synthetic-aperture radar entails the synthesis of multiple measurements taken by a single sensor as if the set of measurements 
were an array of multiple sensors transmitting and receiving simultaneously.

Conventional processing of recorded synthetic-aperture radar signals produces a two-dimensional image where each pixel of the image is strongly influenced by the surface reflectivity and roughness. Synthetic-aperture radar has been employed primarily with microwave radiation in remote sensing applications. Such applications include terrain mapping studies [49-51], monitoring of tree density in forested areas [52-54], and estimating soil moisture content for agricultural purposes [55-57].

THz SA imaging provides another THz imaging modality that extends techniques used from synthetic-aperture radar. Broadband THz SA imaging is unique in that in one 2-D scan, it provides images at multiple depths and does not rely on a narrow time-window for depth isolation. One important distinction and advantage of $\mathrm{THz}$ detection systems, like microwave radar systems, is that they measure electric field quantities directly and are phase coherent, thus supporting imaging modalities such as SA imaging $[58,59]$.

THz SA 3-D imaging can be done purely in the time domain with a delayand-sum approach, or in the frequency domain [60]. SA imaging relies on diffuse scattering from the facets of a presumably inhomogeneous material. Therefore SA imaging is quite robust to scattering effects and is not as sensitive to alignment as other $\mathrm{THz}$ imaging modalities. In addition, broadband $\mathrm{THz} \mathrm{SA}$ imaging has 
been shown to produce considerable depth resolution [61].

THz SA imaging resembles back-projection algorithms for computed tomography that have been explored in the THz range [62-65], which use a transmission configuration. However, many imaging targets such as the human body are opaque to $\mathrm{THz}$ waves, adding significant complications to transmission-based computed tomography. In this dissertation, a $2-\mathrm{D} \mathrm{THz}$ monostatic synthetic planar array is investigated to generate $\mathrm{THz}$ images at multiple depths using broadband averaging. The reflection-mode geometry will likely be better suited for integration into a manufacturable $\mathrm{THz}$ system. It will be shown that 3-D broadband $\mathrm{THz} \mathrm{SA}$ imaging with considerable depth resolution is possible provided there is sufficient system bandwidth. If the system does not have wide enough bandwidth, or if a narrowband system is required, then the object to be imaged must be in the near-field of the array. To first order, that means that the length of the array must be at least the square root of the wavelength times the object distance.

THz phased-array imaging systems do not need to operate with a synthetic aperture, as it will eventually be possible to manufacture physical arrays of $\mathrm{THz}$ antennas that operate simultaneously. However, in an optical pump/probe setup, using a single Thz source to create a synthetic array will increase the signal power at each sensor position, as the laser source will not need to be diverted to multiple 
antennas. In addition, SA imaging can lead to improved lateral resolution over conventional phased-array imaging [66].

\subsection{Spectral imaging and identification}

Spectral imaging systems provide both spatial and spectral information, that can be used to characterize a target. Spectral imaging utilizes the fact that any given material reflects energy that varies as a function of wavelength or frequency. Spectral imaging sensors measure the reflectivity of the materials within each pixel area at multiple spectral bands. Often, spectral imaging is passive in that all the incident radiation comes from natural sources, such as the sun [67].

Although THz spectral imaging is a new area of research, hyperspectral imaging using infrared sensors is an established research area in the field of remote sensing. Using optical intensity sensors in a passive sensing configuration, terrain can be mapped using each geographical features' varying reflectance. For example, different types of vegetation will scatter more or less energy to the sensor as a function of wavelength. A target feature may have a local spectral variation or a broadband reflectance that separates it from the background. Typically these systems operate in several bands, allowing materials to be found based on their spectral fingerprints [68].

THz spectral imaging is a new and emerging field, coinciding with the advent 
of powerful $\mathrm{THz}$ source and receiver pairs, and showing promise in a wide range of fields. However, there has been particular interest in the pharmaceutical and defense industries, as drugs and explosive compounds contain unique spectral signatures in both reflection and transmission.

$\mathrm{THz}$ spectral imaging has largely been achieved by applying developed techniques from traditional hyperspectral imaging. The matched filter is considered one of the most powerful remote sensing spectral imaging techniques [67]. A simplified version of the matched filter has been demonstrated for $\mathrm{THz}$ spectral imaging in a transmission configuration [69]. However, to fully utilize a matched filter, an accurate scattering model must be invoked to "match" the measurement to the chosen situational model. Typically, linear mixing models that assume the scattering coefficient is a linear combination of the various material present in the resolution cell $[70,71]$. The matched filter has been shown to produce optimal SNR gain if the model accurately describes the measurement, provided that the clutter covariance matrix for the additive noise is known [67]. In practice, these items can be difficult to estimate, and target variability caused by random inhomogeneities can cause shortcomings in matched filter performance [72]. Explosive materials commonly contain small volume enclosures and rough surfaces. While such features can complicate detection techniques, they enhance diffuse scattering $[8,73]$, which provides a flexible viewing geometry $[74,75]$. 


\section{Chapter 3}

\section{THz Time-Domain Spectroscopy}

This section outlines basic $\mathrm{THz}$ spectroscopy methods in both transmission mode and reflection mode. This chapter also details the measurement system used in this work, a Picometrix T-Ray 4000, and concludes with a study on scattering effects of derivative spectra at specular and diffuse angles of an explosive simulant, $\alpha$-lactose.

$\mathrm{THz}$ spectroscopy can be done either in transmission mode or reflection mode. Transmission mode is the most common, as the absorption peaks are much more pronounced than spectral features from reflection mode spectroscopy. However, transmission mode requires a laboratory prepared sample that is thin enough to be transparent in the $\mathrm{THz}$ range and cannot have any other opaque objects in the path between transmitter and receiver. Geometries for both transmission mode

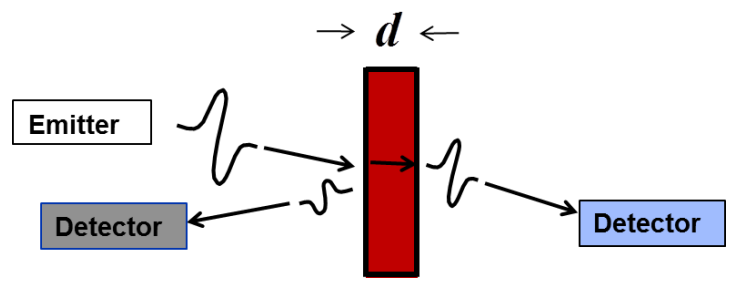

Figure 3.1: Geometry for transmission mode spectroscopy (conventional method; detector shown in blue) and reflection mode spectroscopy (detector shown in gray). 
spectroscopy (conventional method) and reflection mode spectroscopy are shown in Figure 3.1.

Typically, a discrete Fourier transform of the incident pulse is taken to reveal spectral information. The incident time-domain pulse is denoted as $E\left(t_{u}\right)$ and can be generated in a variety of ways. The discrete Fourier transform for a single $\mathrm{THz}$ pulse is given by

$$
E\left(f_{q}\right)=\mathscr{F}\left\{E\left(t_{u}\right)\right\}=\sum_{u=0}^{N_{t}-1} E\left(t_{u}\right) e^{-i 2 \pi \frac{q}{N_{t}} u}
$$

where $f_{q}$ is the $q^{\text {th }}$ frequency point, and $N_{t}$ is the total number of data points included in the time series. A typical $\mathrm{THz}$ pulse measured in free space is shown in Figure 2.4(a). The frequency spectrum of the incident $\mathrm{THz}$ pulse as computed from (3.1) is shown in Figure 2.4(b). The spectral resolution after the discrete Fourier transform is inversely proportional to the width of the incident pulse, which is decided ultimately by a time window. Usually, each pulse is then zeropadded after the time window to improve spectral resolution, although doing this does not add additional information.

In transmission mode, the $\mathrm{THz}$ pulse is transmitted through the sample, and recorded on the other side by a receiver. The electric permittivity describes a material's polarizability in response to an external oscillating electric field. In general, the permittivity is complex and frequency dependent. The relative 
electrical permittivity is given by

$$
\epsilon\left(f_{q}\right)=\epsilon^{\prime}\left(f_{q}\right)-i \epsilon^{\prime \prime}\left(f_{q}\right),
$$

with absorption in the material mostly described by the imaginary part of the permittivity. Since the above permittivity is relative, the actual permittivity can be found by multiplying by $\epsilon_{0}$, or $8.85 \times 10^{-12} \mathrm{~F} / \mathrm{m}$. For this work, the magnetic permeability is assumed to be that of free space.

When the $\mathrm{THz}$ pulse passes through a material at normal incidence with electrical permittivity, $\epsilon\left(f_{q}\right)$, the expected transmitted signal strength for each frequency, is given by

$$
E_{t}=E_{i n}\left(f_{q}\right) T_{s}\left(f_{q}\right) e^{-i k_{s} d}
$$

where $E_{i n}\left(f_{q}\right)$, is the incident pulse spectrum, $d$ is the sample thickness, and $k_{s}$ is the wavenumber inside the sample given by

$$
k_{s}=2 \pi f_{q} \sqrt{\mu \epsilon\left(f_{q}\right) \epsilon_{0}} .
$$

The combined Fresnel transmission coefficient, $T_{s}\left(f_{q}\right)$, for both the front and back surface, is given by 


$$
T_{s}\left(f_{q}\right)=\frac{4 \sqrt{\epsilon_{r}\left(f_{q}\right)}}{\left(\sqrt{\epsilon_{r}\left(f_{q}\right)}+1\right)^{2}},
$$

where all etalon effects (multiple reflections) are ignored. This is a sufficient approximation when the sample is thick enough to time-gate all subsequent reflections. In transmission mode, a target can be identified by selecting narrow frequency bands around absorption peaks. In reflection mode, the situation is more difficult, as there are no longer sharp absorption peaks. The reflected electric field at normal incidence from a thick sample is given by

$$
E_{r}\left(f_{q}\right)=E_{i n}\left(f_{q}\right) R_{s}\left(f_{q}\right),
$$

where $E_{i n}\left(f_{q}\right)$ is the incident pulse spectrum. Again, all multiple reflections have been ignored. The reflection coefficient, $R_{s}\left(f_{q}\right)$, from the sample material is given by

$$
R_{s}=\frac{1-\sqrt{\epsilon_{r}\left(f_{q}\right)}}{1+\sqrt{\epsilon_{r}\left(f_{q}\right)}} .
$$

One may notice by comparing (3.3-3.7) that the absorption spectrum is highly dependent on the imaginary portion of $\epsilon_{r}\left(f_{q}\right)$, while the reflection spectrum depends primarily on the real portion of $\epsilon_{r}\left(f_{q}\right)$, which is simply the square of the index of refraction, $n$. Figure 3.2(a) shows the absorption coefficient for $\alpha$-lactose 


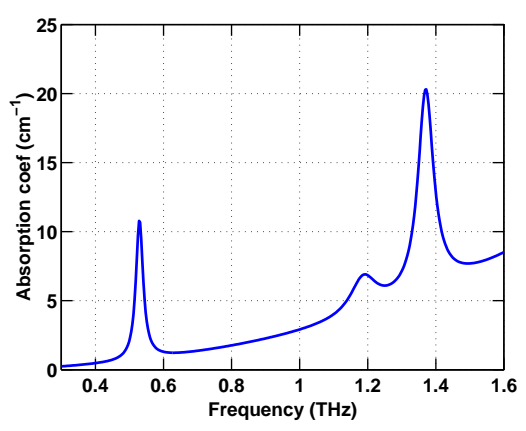

(a)

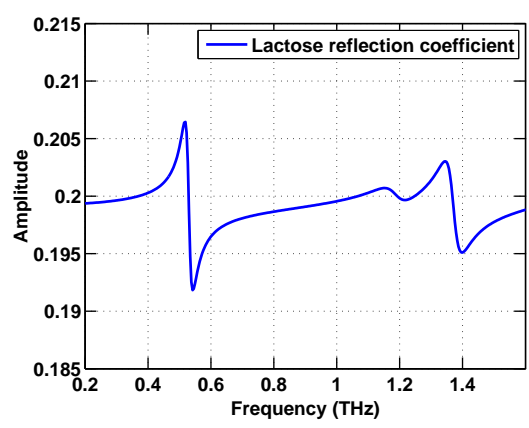

(b)

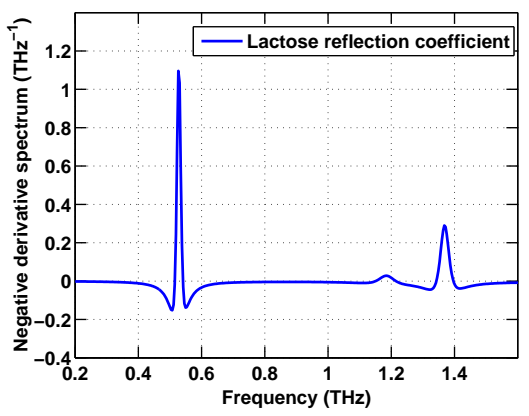

(c)

Figure 3.2: (a) Absorption coefficient for lactose taken from Lorentz parameters, with resonance peaks at $0.53,1.18$, and $1.38 \mathrm{THz}$. (b) Reflection coefficient at normal incidence for lactose using Lorentz parameters, neglecting multiple reflections from within the sample. (c) Derivative spectrum for lactose, showing resonance features at $0.53,1.18$, and $1.38 \mathrm{THz}$.

(will be denoted as simply lactose for the remainder of the dissertation), which has absorption features at $0.53,1.18$, and $1.38 \mathrm{THz}$. Lactose is a powder that is used as a simulant for explosives, and can be pressed into pellets for convenient measurements. Both curves in Figure 3.2 were generated with the Lorentz model for complex permittivity, which includes parameters for width, amplitude, and location of spectral resonances [12]. 


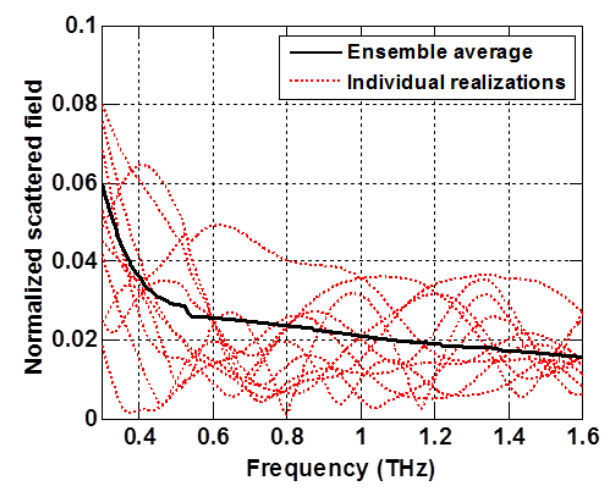

(a)

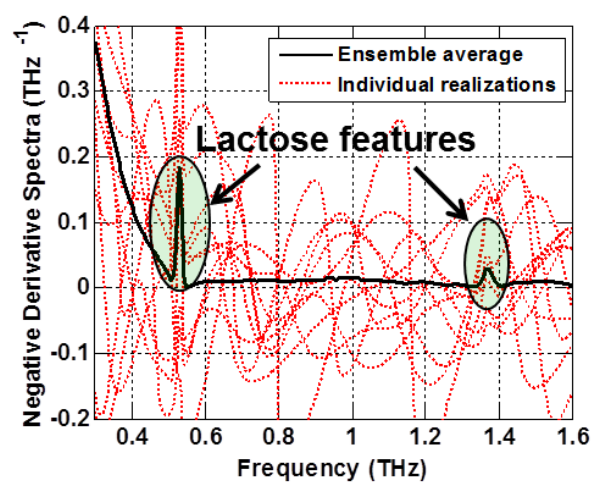

(b)

Figure 3.3: (a) Kirchhoff surface scattering simulation at normal incidence from a rough lactose sample. Red curves represent 10 of the individual realizations, while the ensemble average is shown in black. (b) Negative derivative of simulation showing features at $0.53,1.18$, and $1.38 \mathrm{THz}$.

Figure 3.2(b) shows the reflected amplitude at normal incidence for lactose. As mentioned earlier, one potential method to resolve a target signature in reflection mode is to take the first derivative of the absolute value of reflected amplitude with respect to frequency [21]. The negative derivative, $E_{r, d f}^{\prime}$, can be written as

$$
E_{r, d f}^{\prime}=-\frac{d}{d f}\left|E_{r}\right|
$$

The negative derivate spectrum for lactose is plotted in Figure 3.2(c). Clearly visible in this plot are the lactose features at $0.53,1.18$, and $1.38 \mathrm{THz}$. The negative sign is used in (3.8) so the resonance features are viewed as 'peaks.'

To illustrate the utility of the negative derivative, refer to Figure 3.3(a), which shows the simulated scattered electric field from a rough surface comprised of 
lactose. This simulation was produced using the Kirchhoff (or tangent plane) approximation with a transceiver co-located normal to the surface mean. More details about this type of simulation are provided in Section 4.5. Individual realizations are plotted in dashed red, while the ensemble average is plotted in solid black. There is a global downward trend as a function of frequency, which occurs because the surface appears more rough at shorter wavelengths (higher in frequency). One benifit of taking the negative derivative is that it acts as a highpass filter, removing this trend. Figure 3.3(b) shows the negative derivate spectra, as computed from (3.8). Figure 3.3(b) accentuates each resonance feature and filters unwanted global fluctuations.

\subsection{Picometrix T-Ray 4000 pulsed THz system}

The Picometrix T-Ray 4000, shown in Figure 3.4(a), is a commercially available pulsed time-domain $\mathrm{THz}$ source. It operates by splitting pulses from a mode-locked 100 femtosecond (fs) fiber laser operating at $1.064 \mu \mathrm{m}$ wavelength into a pump beam and a probe beam. The pump beam is used in the transmitter to excite carriers in a photoconductive bow tie antenna held under a DC bias. The excited carriers create brief pulses in current across the antenna with each incident laser pulse. These short current pulses have a rise time on the order of picoseconds, resulting in a $0.2-3 \mathrm{THz}$ frequency spectrum. 


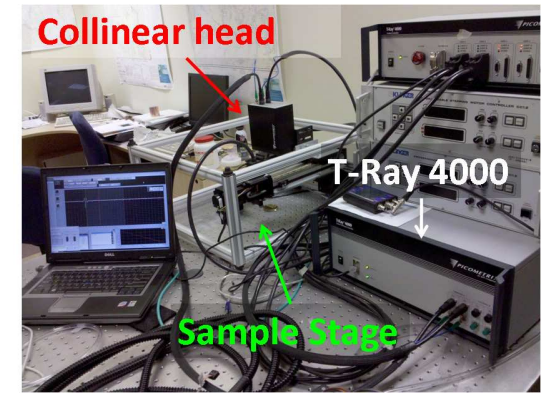

(a)

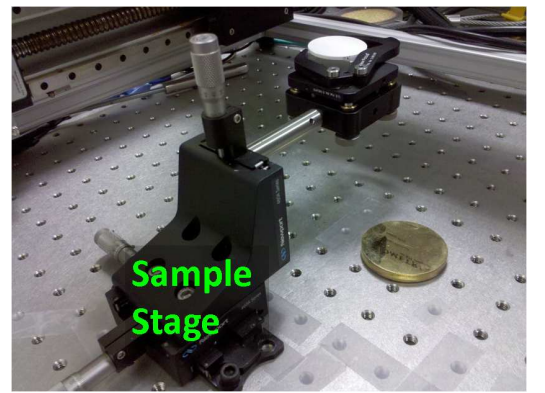

(b)

Figure 3.4: (a) T-Ray 4000 time-domain spectroscopy system shown configured for reflection measurements with collinear head. (b) Close-up of reflection sample stage.

The antenna is affixed to a hyper-hemispherical silicon lens, which directs the energy toward a polyethylene collimating or focusing lens. The receiver consists of a similar set of lenses that focus the incoming $\mathrm{THz}$ pulse into another photoconductive antenna. After passing through a delay line, the probe beam excites carriers in the receiving antenna substrate, which are proportional to the strength of the field. Sweeping the delay of the probe beam with respect to the pump beam samples the incoming $\mathrm{THz}$ pulse at various time points, creating the time-domain waveform.

The Picometrix T-Ray system can be configured for either monostatic measurements (in which the transmitter and receiver are co-located) or bistatic measurements (in which the transmitter and receiver are separate). The collinear head is shown in a reflection measurement configuration in Figure 3.4(a) along with a close-up of the sample stage shown in Figure 3.4(b). 

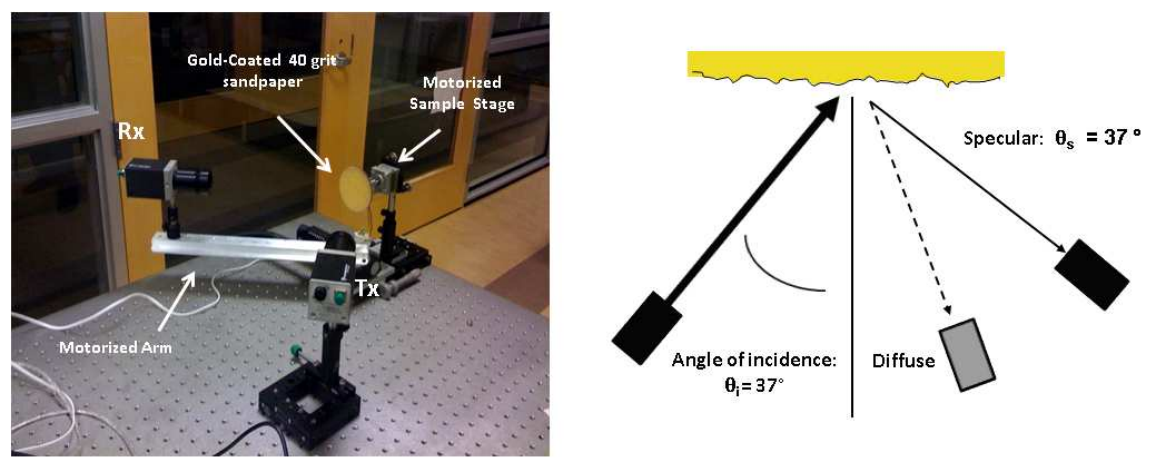

Figure 3.5: (left) T-Ray 4000 transmitter and receiver mounted on a motorized rotation stage. The $\mathrm{THz}$ beam was aligned off-center so that multiple realizations could be generated by rotating the sample. (right) Receiver mounted at the specular angle (solid black) and diffuse (grey).

\subsection{Effects of scattering on lactose reflection signature}

A study was conducted on scattering effects of derivative spectra at specular and diffuse angles near the lactose $0.53 \mathrm{THz}$ resonance feature. All measurements were completed using the T-Ray 4000 system. In order to measure angle-dependent scattering, the transmitter and receiver were used in a bistatic geometry. The transmitter was mounted at $37^{\circ}$ degrees relative to normal incidence to the rough sample. The receiver was mounted on a motorized rotation stage automated by a CC1 Klinger motion controller. The transmitter, receiver, sample stage and motorized arm can be seen in Figure 3.5. Both transmit and receive antennas were focused through collimating lenses. The transmitted beam was approximately one-inch in diameter.

Sample pellets were created by mixing lactose with polytetrafluoroethylene 
Table 3.1: List of sandpaper statistics

\begin{tabular}{|c|c|c|c|}
\hline & 40 grit & 80 grit & 120 grit \\
\hline Correlation Length $(\mu \mathrm{m})$ & 420 & 151 & 156 \\
\hline RMS Height $(\mu \mathrm{m})$ & 135 & 55 & 40 \\
\hline
\end{tabular}

(PTFE) and pressing into a two-inch cylindrical pellet with a 20/80 PTFE/lactose ratio. Samples were prepared by the Naval Surface Warfare Center [76], and are shown in Figure 3.6. The sample thickness, approximately $9 \mathrm{~mm}$, is large enough so that all internal reflections can be time-gated out without significant loss of frequency resolution. Three samples were pressed with 40, 80, and 120 grit sandpaper. A fourth sample was smooth. Table 3.1 shows approximations for root-mean-square (RMS) height, $h$, and correlation length, $l_{c}$ for the various grit sizes of sandpaper. The RMS height is a measure of vertical roughness of the zero-mean surface profile, while the correlation length is the horizontal distance at which the autocorrelation of the surface profile goes to $e^{-1}$. Statistics from
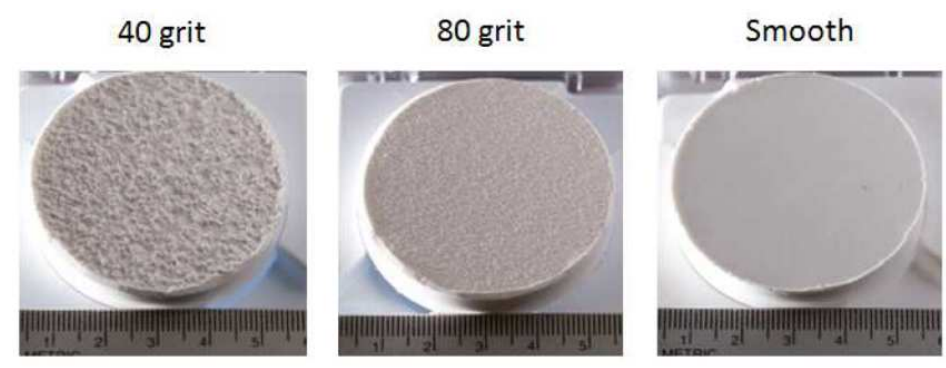

Figure 3.6: Photos of three lactose samples with surface roughness corresponding to 40 grit and 80 grit sandpaper, and a smooth sample. 


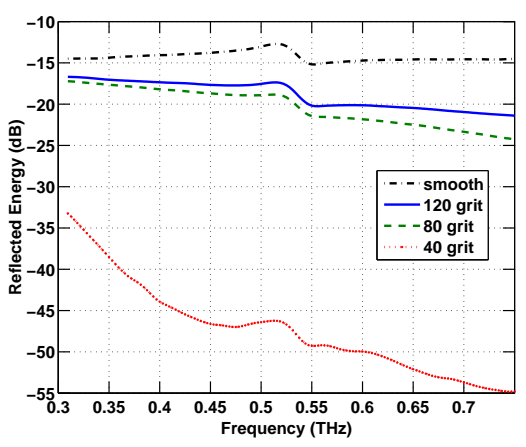

(a)

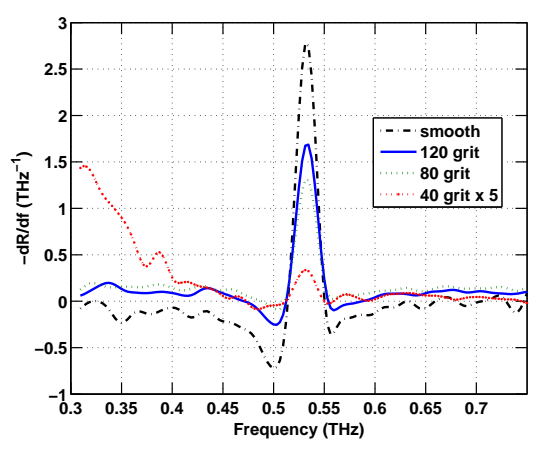

(b)

Figure 3.7: (a) Reflection spectra for 40 grit, 80 grit, and 120 grit samples from lactose, as well as a smooth sample. Each measurement is deconvolved with response from an optically smooth gold reference. (b) Derivative of each curve with respect to frequency.

40 grit and 120 grit sandpaper were extracted from surface profiles measured by Nanovea [9,77]. Statistics for 80 grit sandpaper were taken from [78].

Specular measurements were taken for the smooth, 40, 80, and 120 grit lactose samples. Reflections were recorded for 30 different lateral positions across each sample, and averaged incoherently. After averaging, the reflection from each sample was normalized by a reference measurement taken from a gold mirror to remove incident pulse spectrum and propagation effects. Figure 3.7(a) shows the reflected spectrum from each sample; in each curve the lactose reflection feature is visible at $0.53 \mathrm{THz}$. In order to rectify the absorption peak at $0.53 \mathrm{THz}$, the negative derivative of the each reflection spectrum was taken with respect to frequency, as in (3.8), and is shown in Figure 3.7(b). 
There are a couple of important observations from Figure 3.7. Increasing surface roughness causes a lower baseline reflection spectrum. That is, the roughness lowers the overal reflected energy, which in turn lowers the strength of the absorption peak. For example, the 40 grit sample has $30 \mathrm{~dB}$ less reflected energy than the 120 grit. All grit sizes contain a frequency dip at $0.53 \mathrm{THz}$ due to the resonance feature. But the 40 grit sample has a much lower baseline value. The negative derivative also reveals absorption peaks for all samples, as shown in Figure 3.7(b). However, the rougher the surface, the smaller the absorption feature becomes after taking the negative derivative.

Another interesting thing to note from Figure 3.7(b) is there is a scattering induced slope that is apparent in all grit sizes. Each rough sample displays a downward slope with respect to frequency, which becomes a positive offset in Figure 3.7(b). The 40 grit sample, in particular, shows a rapid decline in both reflection and derivative spectra around $0.35 \mathrm{THz}$. The reflected energy becomes incoherent when the surface is very rough with respect to wavelength. Incoherent energy has a relatively flat spectrum, which can be advantageous in fingerprinting chemicals.

Next, diffuse reflections for the 40 grit lactose sample were obtained in a similar manner. The transmitter was fixed with $\theta_{i}=37^{\circ}$; and the receiver was mounted on the motorized arm shown in Figure 3.5. The scattered angle, $\theta_{s}$, 


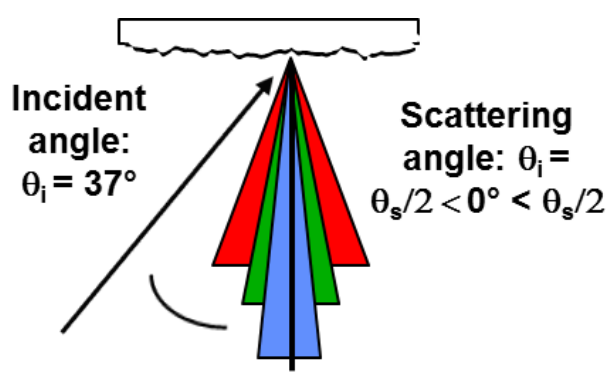

Figure 3.8: Diagram of incoherent average across scattering angles. The blue, green and red angular extent corresponds to angular extents of $4^{\circ}, 16^{\circ}$ and $32^{\circ}$.

ranged from $-16^{\circ}$ to $16^{\circ}$ in increments of $2^{\circ}$ for a total of 17 measured angles.

The measured response from each angle was averaged incoherently from $-\theta_{e} / 2$ to $\theta_{e} / 2$, as shown in Figure 3.8. The averaged field can be written as

$$
E_{\text {average }}(f)=\int_{\frac{\theta_{e}}{2}}^{\frac{\theta_{e}}{2}}\left|E_{s}\left(f_{q}, \theta_{s}\right)\right| d \theta_{s}
$$

where $\theta_{e}$ is the full angular extent. Figure 3.9(a) shows the diffuse scattered amplitude as a function of frequency, as calculated from (3.9). Figure 3.9(b) shows the negative derivative spectrum for 40 grit impressed lactose, with each curve using a different angular extent. For example, the 16 degree curve (solid line) in Figure $3.9(\mathrm{~b})$ is the averaged response from $-8^{\circ}$ to $8^{\circ}$. Thirty surface realizations were recorded for each angular position.

Just as the surface has a correlation length in terms of distance, the scattered field becomes uncorrelated with a sufficient angular separation [9]. For 


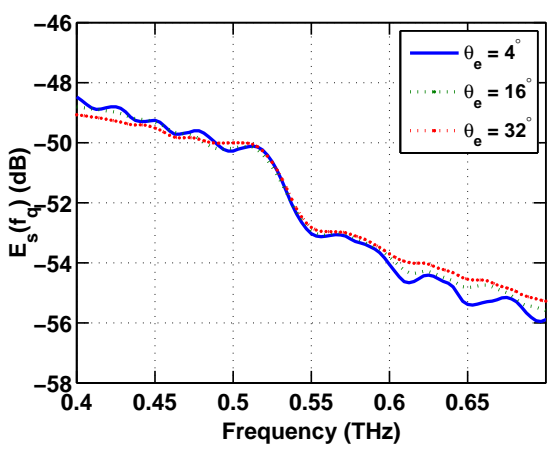

(a)

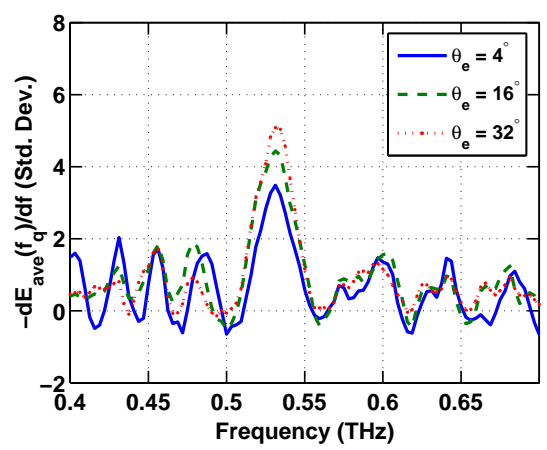

(b)

Figure 3.9: (a) Diffuse reflection spectra for 40 grit impressed lactose, each using different angular extents centered at $\theta_{s}=0^{\circ}$. (b) Derivative spectra for three different angular extents. Each spectrum is normalized so that it contains unity standard deviation.

this reason, averaging many angles removes random variations due to the random rough surface, thus increasing peak-to-noise ratio. Figure 3.9(b) shows the derivative spectrum normalized so that each curve contains unity standard deviation. Clearly, the more angles included in (3.9), the better the resonance feature looks. Considering that the scattered field becomes uncorrelated across multiple sensor positions, SA imaging is a desirable configuration, providing a robust viewing geometry for diffuse returns. SA imaging utilizing multiple sensor locations and viewing geometries will be discussed further in Chapter 5. The next chapter deals with robust chemical detection techniques. 


\section{Chapter 4}

\section{Robust Chemical Detection Techniques}

This chapter describes three robust methods of detecting a resonance peak, and validates them with imaging and simulation results. The matched filter, which has been used in conventional hyperspectral imaging, is described first [71]. Then, two new detection methods, namely the squared residual method and correlation processing method, which were developed as part of this dissertation, are described. Both methods seek to minimize the effect from reflections off background materials that have relatively flat spectral characteristics. That is, each method tests the hypothesis of constant reflected magnitude or phase in regions of the spectrum where a resonance feature from a target material is expected. Many packaging materials, such as plastics, contain such flat spectral magnitude and phase across a wide range of $\mathrm{THz}$ frequencies.

\subsection{Matched filter detection}

As shown in Chapter 3, the negative derivative helps to identify a resonance feature, and also acts as a high pass filter. While this helps to remove global trends, it also means that high frequency noise is amplified. This section outlines 
another method that is used to detect resonance feature, namely the matched filter. The matched filter is commonly used in infrared spectral imaging, and can represent significant improvement over the negative derivative. The matched filter provides optimal SNR gain in the case where the target signature is known exactly and mixed with solely additive white Gaussian noise [71].

Suppose a spectral reflection measurement is taken from an imaging sensor with $K$ frequency bands. The complex spectral measurement is written as

$$
X=\left[x_{1} x_{2} x_{3} \ldots x_{K}\right]^{T}
$$

An optimal matched filter can be written as

$$
Y=H^{T} X
$$

where $H$ is given by

$$
H=\Sigma^{-1} s_{0}
$$

where $s_{0}$ is the model for the target signature. $\Sigma^{-1}$ is the clutter covariance matrix for the additive noise. The superscript, ${ }^{T}$, denotes conjugate transpose, or Hermitian transpose. In the case of white Gaussian noise, $\Sigma^{-1}$ reduces to the identity matrix, and $H$ becomes simply $s_{0}$. In practice, the clutter covariance 
matrix, $\Sigma^{-1}$, can be difficult to estimate.

Given the nature of explosive devices, which can be constructed of many materials and packed in various ways, a robust detection method is strongly desired. While a matched filter has been proven to maximize SNR in the presence of additive Gaussian noise, an accurate model must be known. In addition, if there is too much spectral variation inherent in a target feature, or the noise covariance matrix is not known, the ability of a conventional matched filter to detect a material will be diminished [72]. While matched filter detection is not a new method, it has not been thoroughly investigated for chemical identification in the $\mathrm{THz}$ region.

\subsection{Correlation processing}

Most current detection systems rely solely on magnitude of scattered signals, throwing away important phase signatures [21]. While the reflected phase from such a compound does contain unique signatures, looking at the phase directly can lead to noise and unwrapping errors.

Correlation processing has been used in radar applications for suppressing background surface clutter by self-correlating the electric field across frequency with a reference function [79]. In this work, correlation processing is modified to suppress reflections from materials with constant spectral phase by correlating 


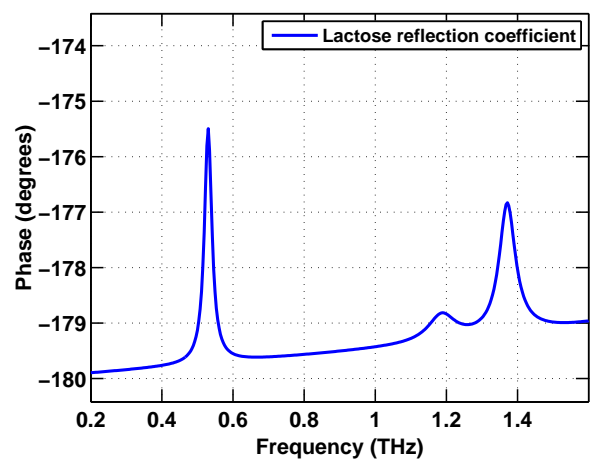

Figure 4.1: Phase of the reflection coefficient at normal incidence from lactose, and explosive simulant.

the electric field with a phase-structured field. For use in $\mathrm{THz}$ chemical detection, correlation processing can be applied in particular areas of the spectrum where a phase resonance is expected.

Figure 4.1 shows the phase of the reflection coefficient from lactose at normal incidence. The three lactose peaks at $0.53,1.18$, and $1.38 \mathrm{THz}$ are visible. While it is problematic to use the phase directly to detect the material, the phase can still provide meaningful information. By sampling the phase in the frequency domain near a resonance, and correlating the observed field, $E\left(f_{q}\right)$, with a phasestructured reference field, one can test the hypothesis of rapidly changing phase. Materials that have sharp phase features in the chosen frequency band will contain a higher value relative to spectrally flat materials. The correlation processing output, $C P O$, for a single spectral region of interest, is defined as 


$$
C P O=\frac{1}{Q} \sum_{q=1}^{Q} E\left(f_{q}\right) \times E_{C P}\left(f_{q}\right),
$$

where $\Delta f$ is the frequency resolution, and $Q$ is the total number of frequency points in the band. The $q^{\text {th }}$ frequency bin, $f_{q}$, can be written as

$$
f_{q}=f_{1}+q \Delta f .
$$

The phase-structured field, $E_{C P}\left(f_{q}\right)$, can be written as

$$
E_{C P}=\exp \left(i \alpha f_{q}\right) .
$$

The phase-structured field adds phase to $E\left(f_{q}\right)$ in (4.4) and scales proportionally with frequency. The phase slope, $\alpha$, is defined as

$$
\alpha=\frac{2 \pi}{\Delta f Q} .
$$

The phase slope is chosen so that $e^{i \alpha f_{q}}$ has $2 \pi$ radians phase difference between the first frequency in the band, $f_{1}$, and the last, $f_{1}+Q \Delta f$. In other words, the average value of $E_{C P}\left(f_{q}\right)$ is defined to be zero,

$$
\frac{1}{Q} \sum_{q=1}^{Q} E_{C P}\left(f_{q}\right)=0 .
$$




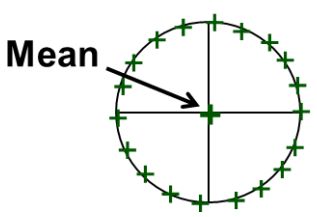

$\mathrm{H}_{0}$ : Constant phase CPO $<$ THRESHOLD

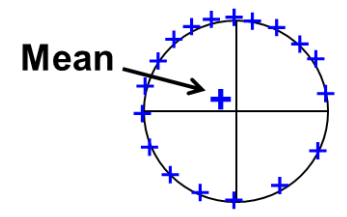

$\mathrm{H}_{1}$ : Phase feature present CPO > THRESHOLD

Figure 4.2: Illustration of correlation processing terms plotted on complex circle for spectrally flat material (left; terms plotted as green plus's) and one that contains a feature (right; terms plotted in blue). The coherent average is nonzero for materials with a phase feature, and becomes close to zero for materials with spectrally flat phase.

Therfore, after the evaluation of (4.4), any material with flat spectral phase in this band would approach zero, while materials with sharp features across this same band would become nonzero. To illustrate the mechanics of this technique, the individual terms in (4.4) are plotted on the complex circle in Figure 4.2. That is, each plus represents a single frequency in the summation. First, terms from a spectrally flat reflection are plotted (left), which are equally spaced around the perimeter of the circle. The coherent average of these terms should approach zero, depending on the signal-to-noise ratio. Next, terms are plotted from a material that has a phase feature in the region under test (shown right), which appear clustered on one side, forcing a higher value after taking the coherent average. To detect a specific chemical, the correlation processed reflection data in each spectral region would be compared to values retrieved from a library containing 
a variety of target materials. For a single resonance feature, it makes sense to apply correlation processing in two regions, one on either side of the main peak.

\subsection{Squared residual detection}

Correlation processing discussed a method to minimize the reflection from a material with flat spectral phase. This section outlines a robust method to minimize the reflection from a background material using amplitude fluctuations while enhancing returns from target material. To do this, a polynomial regression fit is taken of the reflected amplitude using the method of least squares. The polynomial fit primarily serves to remove any global trend in the spectra produced by scattering. Specular reflections from rough surfaces will generally contain a downward global trend, as the surface appears rougher at higher frequencies. Conversely, diffuse reflections from rough surfaces will generally contain an upward trend as a function of frequency [74]. However, these trends can be removed by the fitting process. In practice, the least squares polynomial fit may be of arbitrary order. The residuals from the least squares fit are squared and summed. The squared residuals, $S R$, for a single feature are given by

$$
S R=\frac{1}{Q} \sum_{q=1}^{Q}\left[\left|E\left(f_{1}+q \Delta f\right)\right|-\left|E_{f i t}\left(f_{1}+q \Delta f\right)\right|\right]^{2} .
$$


Figure 4.3(a) shows an example the reflected spectrum from a rough sample of lactose at normal incidence (in blue). There is a noticeable downward trend as a function of frequency. Also plotted in Figure 4.3(a), is the polynomial fit, shown in green. Figure 4.3(b) shows the squared residuals by subtracting the polynomial fit as in (4.9) before averaging across frequency. The squared residuals should approach zero for spectrally flat materials. As with correlation processing, squared residuals in each spectral region can be compared to library values for various target materials.

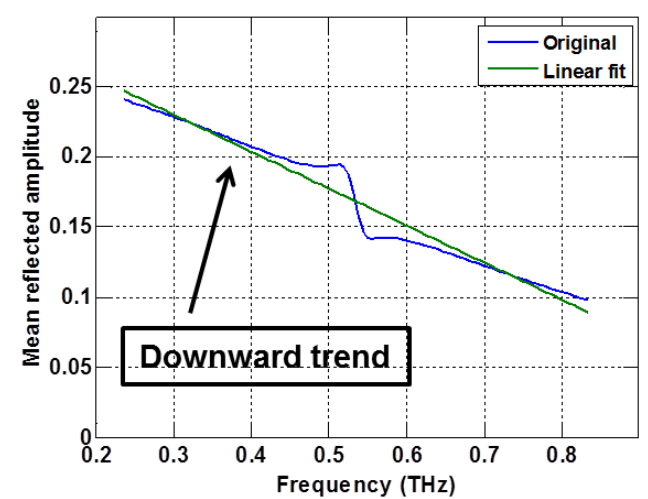

(a)

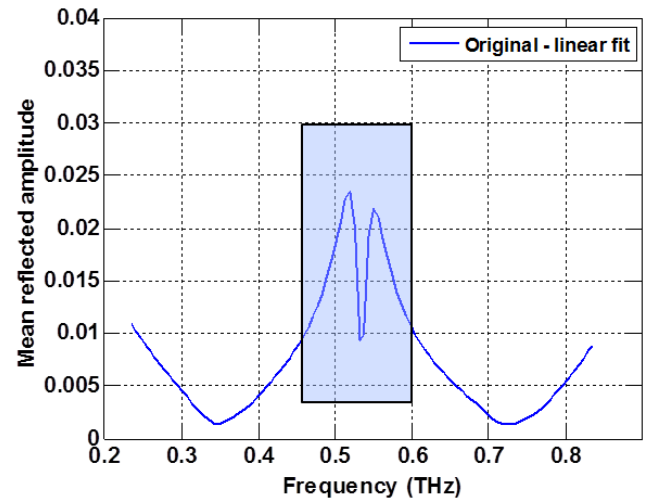

(b)

Figure 4.3: (a) Reflected amplitude from a rough surface made of lactose. A downward trend is apparent due to rough surface scattering. (b) Squared residuals from (4.9) before averaging across frequency.

\subsection{Validation of detection techniques}

This section validates the various detection algorithms in an imaging configuration. In order to perform this validation and comparison, THz images of a 


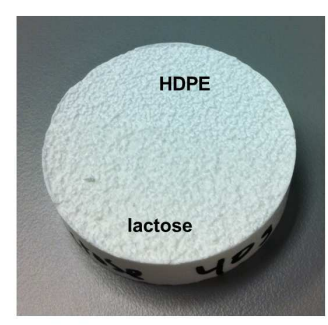

(a)

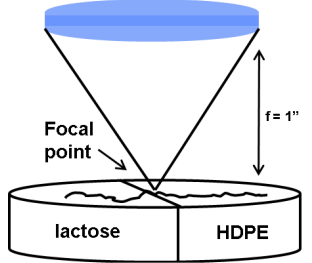

(b)

Figure 4.4: (a) Optical image of a two-sided pellet constructed with one side containing lactose and the other side pure high-density polyethylene with 40 grit rough sandpaper impressed. (b) Imaging configuration using a T-Ray 4000 pulsed $\mathrm{THz}$ system with the pellet sitting in the focal plane of a one-inch polyethylene focusing lens.

two-sided pellet are produced to show the chemical mapping capability in a realworld imaging scenario with chemical mixtures and a rough surface, each of which produce scattering effects. All measurements were conducted in the NEAR-Lab using the T-Ray $4000 \mathrm{THz}$ pulsed imaging system, described in Section 3.1. All techniques in this dissertation have been implemented using MATLAB.

\subsubsection{Imaging results using two-sided pellet}

A pellet was constructed out of lactose and HDPE with 40 grit sandpaper impressed to roughen the surface. One side of the pellet is $75 \%$ lactose mixed with $25 \%$ HDPE, by weight. The other side is pure HDPE. The pellet was made in a hydraulic press, and is shown in 4.4(a). The pellet surface was placed in the focal plane of a one-inch monostatic focusing lens, as shown in Figure 4.4(b). 


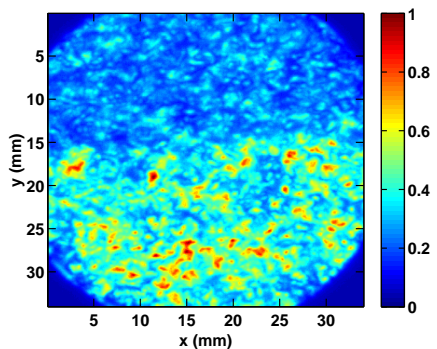

(a)

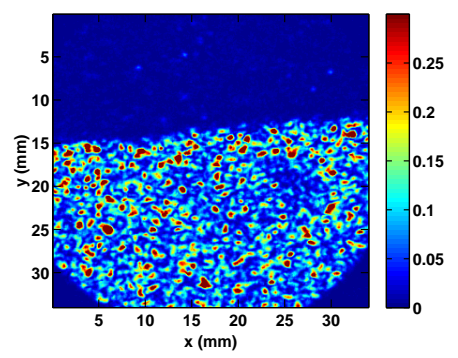

(c)

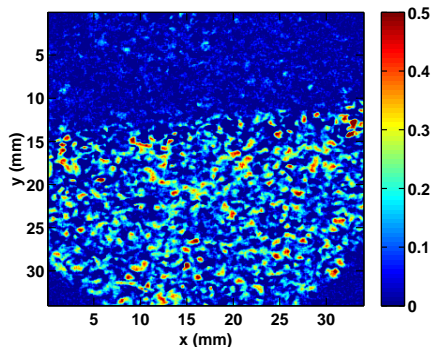

(b)

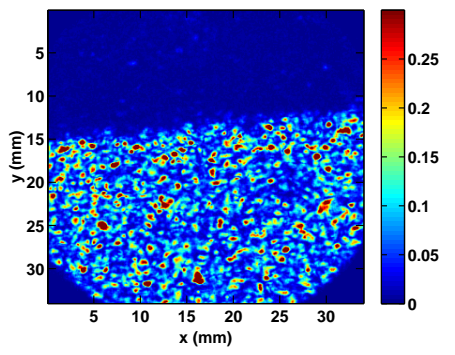

(d)

Figure 4.5: (a) Broadband $\mathrm{THz}$ reflectance image of a pellet with the top half consisting of lactose and the bottom high-density polyethylene with 40 grit rough sandpaper impressed. The colorscale is normalized by the maximum pixel in the image. (b) Derivative image as computed from (3.8) of pellet taken at 0.53 THz. (c) Correlation processing image, as computed from (4.4) using only the first lactose peak, two separate spectral regions $0.5-0.53 \mathrm{THz}$ and $0.53-0.56$, and summing contributions from each region incoherently. (d) Squared residual image of the pellet calculated between 0.5 and $0.58 \mathrm{THz}$ using (4.9). 
Figure 4.5(a) shows an image of the pellet described above using the broadband $\mathrm{THz}$ reflectance to populate each pixel value or color. For comparison purposes, an image showing the negative derivative as calculated in (3.8) is also shown in Figure 4.5(b), and does reveal a difference between the two peaks. The colorscale corresponding to each image is normalized by maximum pixel in the image.

Figure 4.5(c) shows an image of a pellet described above using $C P O$ from (4.4) to compute each pixel value. This image was computed using only the first lactose peak, two separate spectral regions $0.5-0.53 \mathrm{THz}$ and $0.53-0.56$, and summing contributions from each region incoherently. The bottom half of the pellet, the lactose side, is much brighter in the false color image. The two sides are much easier to distinguish than in Figure 4.5(b). Figure 4.5(d) shows a similar image using the squared residual method from (4.9) to populate each pixel value. As with Figure 4.5(c), one can clearly distinguish between the two sides. An important observation is that it's difficult to see the top edge of the pellet in each figure. In other words, the reflections from the HDPE have been completely canceled and have gone into the noise level, which is the intended result for both $C P O$ and $S R$. Qualitatively, these two imaging/detection techniques perform very well in comparison to the more established derivative method.

Although the techniques are similar, there is a distinction between Figures 4.5(c) 
and $4.5(\mathrm{~d})$. There are a few bright sections in Figure 4.5(d) that do not appear in Figure 4.5(c), implying that these images can provide complimentary information. Perhaps some scattering artifacts are driven by phase, while others are influenced more by scattered amplitude. In an attempt to answer this question, correlation processing and squared residual values were manually selected from a portion of the lactose side and a portion of the HDPE side of the pellet.

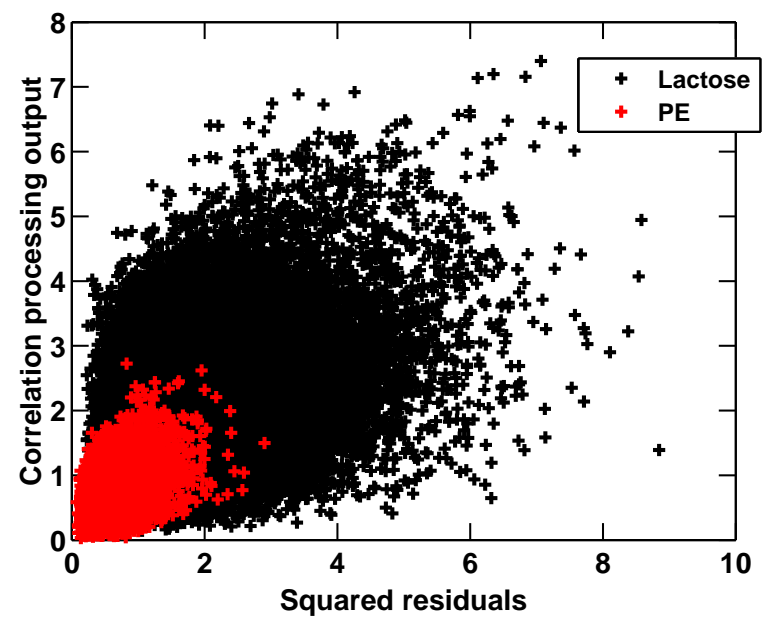

Figure 4.6: Plot of the value of $C P O$ (y-axis) vs. $S R$ ( $x$-axis) for individual pixels in each region, with lactose region data in black and $\mathrm{PE}$ region data in red.

Figure 4.6 shows the value of $C P O$ (y-axis) vs. $S R$ ( $x$-axis) for individual pixels in each region, with values from the lactose region in black and HDPE region in red, with both axis values normalized to their standard deviations. If there were strong correlation between $C P O$ and $S R$, this image would form an elliptical cluster of points, which is not the case. Thus, correlation processing and squared residuals are somewhat independent and can be complementary methods, 
particularly in the case of the confounding material, HDPE. Because of this independence, both methods can be combined to provide an enhanced detection method, complimentary feature detection, denoted $C F D$, which is described as mathematically as

$$
C F D=C P O+\gamma S R
$$

where $\gamma$ is a constant. One can think of combining both techniques as applying a linear threshold to both populations in Figure 4.6, with the slope of the threshold being determined by $\gamma$.

\subsubsection{Performance metrics}

To quantify the ability to correctly identify a material with a resonance feature (such as lactose), each detection metric is compared to a set threshold, marking one of two decisions; detection of chemical or no detection. In the case of correlation processing, the probability of detection, $P_{d}$, can be defined as probability of $C P O$ exceeding $\gamma$, given that the material in question is indeed lactose. That is,

$$
P_{d}=P(C P O>\gamma \mid \text { lactose })
$$

Conversely, $P_{f a}$, the probability of false alarm, can be defined as the probability 
of $C P O$ exceeding $\gamma$, given that the material in question is actually HDPE. That can be written as

$$
P_{f a}=P(C P O>\gamma \mid \mathrm{HDPE})
$$

Clearly, both $P_{d}$ and $P_{f a}$ are strongly dependent on the choice of $\gamma$. By sweeping the threshold, $\gamma$, from a small number to a large number, receiver operating characteristic (ROC) curves can be generated. ROC curves typically plot the probability of detection, $P_{d}$, against probability of false alarm, $P_{f a}$, and are considered one of the standard ways to analyze the performance of a detection algorithm. An ideal ROC curve is contained in the upper left quadrant, achieving $100 \%$ chance of detection, while maintaining a $0 \%$ false alarm rate. More information about the generation of ROC curves is provided in Appendix B. In order to plot ROC curves corresponding to the processed pellet data, sections of scattered data were manually selected from the top and bottom half of the 2-sided pellet measurements shown in Figure 4.5. For each sample realization (or pixel), the $C P O, S R$, or negative derivative was compared with $\gamma$ to decide whether or not lactose was present.

For the following ROC study, reflected data from each population (HDPE and lactose) were manually selected from both the top and bottom half of the 2-sided pellet images in Figure 4.5. For each sample realization (pixel), a decision 


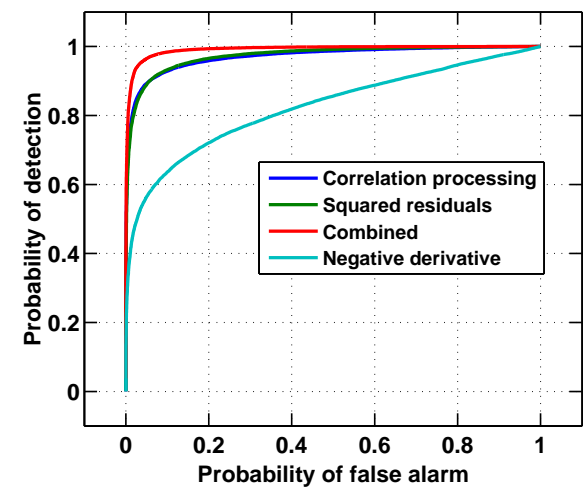

(a)

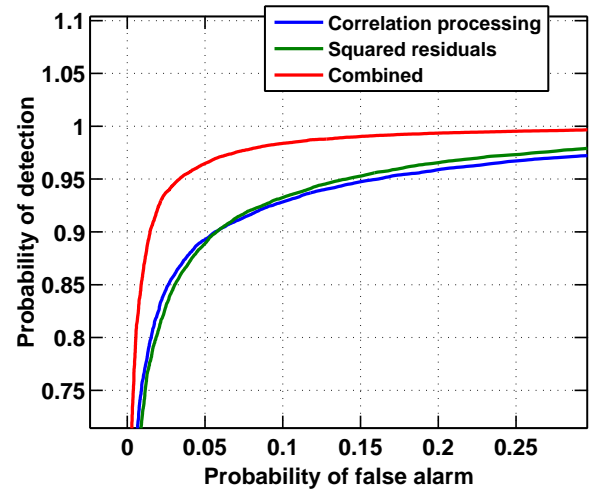

(b)

Figure 4.7: (a) Receiver operating characteristic curve produced from data measured from the two-sided pellet consisting of lactose and high-density polyethylene. (b) Receiver operating characteristic curve produced from the same data, with CFD as computed in (4.10) in red.

is made on whether the target, lactose, is present.

Figure 4.7(a) shows three ROC curve corresponding to the $C P O, S R$, and negative derivative-based decisions as computed from (4.4), (4.9), and (3.8) respectively, in addition to $C F D$ from (4.10). The $C P O$ and $S R$ curves perform the best, providing a $P_{d}$ value of roughly 0.87 , while maintaining a $P_{f a}$ of 0.05 . Figure 4.7(b) shows both curves from Figure 4.7(a), while constraining $P_{f a}$ to less than 0.3. By combining both $C P O$ and $S R, P_{d}$ increases from 0.87 to 0.93 while keeping $P_{f a}$ constant at 0.05 . 


\subsection{Monte Carlo Kirchhoff simulations}

In order to assess the utility of various detection techniques for $\mathrm{THz}$ imaging of explosive devices, a Monte Carlo simulation was conducted. A Monte Carlo simulation of this nature using numerically generated rough surfaces is a useful tool to quickly predict scattering behavior from a rough surface with chosen roughness parameters and dielectric constants. It also allows for a quick way to test and compare the various detection techniques.

In this section, the Kirchhoff (or tangent plane) approximation was used to calculate the scattered electric field from 1,000 numerically generated random surfaces. The backscattered electric field was calculated for each realization using both a known target material, lactose, C-4 and a confounding material with similar reflectance. Then, the scattered electric field from each realization was applied to a detection algorithm such as (4.4) to decide which population that rough surface belongs.

First, details are provided on how the scattered field is computed from a onedimensional surface, following closely from $[9,80]$. Huygens Principle may be used to sum the scattered fields from all the points along a rough surface to give the total scattered field, $E_{s}$, at a point far above the rough surface. The Kirchhoff approximation then treats the facets of the rough surface as tangent planes and applies the Fresnel reflection coefficient to calculate the local scattered fields. The 
total scattered field from a one-dimensional rough surface extending from $-L$ to $L$ is given by [80]

$$
E_{s}^{ \pm}=\frac{\mp 1}{4 L \cos \theta_{i}} \int_{-L}^{L}\left(a \delta^{\prime}(x)-b\right) e^{i v_{x}+i v_{z} \delta(x)} d x,
$$

where $E_{s}$ is the scattered field from a rough surface, normalized by the reflected field from a perfectly smooth conducting surface. The notation, $(+,-)$, represents vertical and horizontal polarization states, respectively. In $(4.13), \delta(x)$ is the surface profile as a function of horizontal position $x$, and $\delta^{\prime}(x)$ is the first-order derivative of the surface profile with respect to $x$.

The phase shift due to surface roughness, $\delta(x)$, is dictated by the exponential terms, $v_{x}$ and $v_{x}$, which depend on transceiver location, which is in this case is at normal incidence relative to the surface mean. The electrical permittivity of the surface, $\epsilon_{s}$, is accounted for in the coefficients $a$, and $b$.

Although the integral in (4.13) is difficult to solve analytically, it can be solved numerically for a computer-generated rough surface. Realizations of Gaussian random rough surfaces can be generated with a given RMS height, $h$, and correlation length, $l_{c}$. The scattered field depends on the total length of the illuminated surface, $2 L$, which typically spans many wavelengths. Simulations in this paper used $2 L=4 \mathrm{~mm}$. 


\subsubsection{Case \#1: lactose (target) versus $\mathrm{HDPE}$}

In the first study, the various detection techniques were applied to lactose in the presence of a confounding material, namely HDPE (index of refraction, $n$, was assumed to be 1.55). 1,000 numerical surfaces were generated for each material from 0.4 to $0.7 \mathrm{THz}$ with spacing of $6.25 \mathrm{GHz}$, the same frequency resolution from measurements. Only the first lactose resonance peak at $0.53 \mathrm{THz}$ was used in detection. Ultimately, receiver operating characteristic (ROC) curves were calculated using two populations, scattered electric field from lactose and HDPE.

The first simulation used an RMS surface height, $h$, of $43 \mu \mathrm{m}$ and correlation length, $l_{c}$, of $480 \mu \mathrm{m}$, a surface that is relatively smooth yet still produces a significant amount of scattering according the Fraunhofer criterion. Gaussian noise was added with SNR of $30 \mathrm{~dB}$. Figure 4.8(a) shows ROC curves corresponding to correlation processing, matched filter, squared residual and negative derivative-based decisions are in blue, green, red, and cyan, respectively. Correlation processing was calculated from (4.4) using only the first lactose peak, two separate spectral regions $0.5-0.53 \mathrm{THz}$ and $0.53-0.56$, and summing contributions from each region incoherently.

The second simulation used a surface height of $135 \mu \mathrm{m}$, a correlation length of $420 \mu \mathrm{m}$, corresponding to the surface statistics of 40 grit sandpaper, and no additive noise. ROC curves corresponding to this simulation is shown in 
Figure 4.8(b). For the matched filter detection in each plot, a polynomial fit was subtracted from each reflection spectrum, resulting in zero-mean lactose and HDPE spectra.

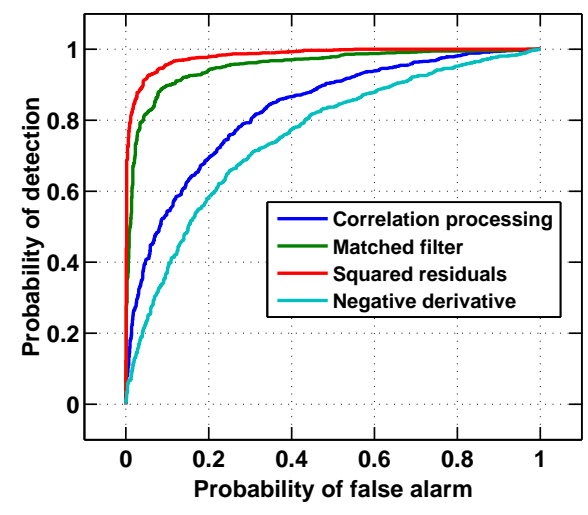

(a)

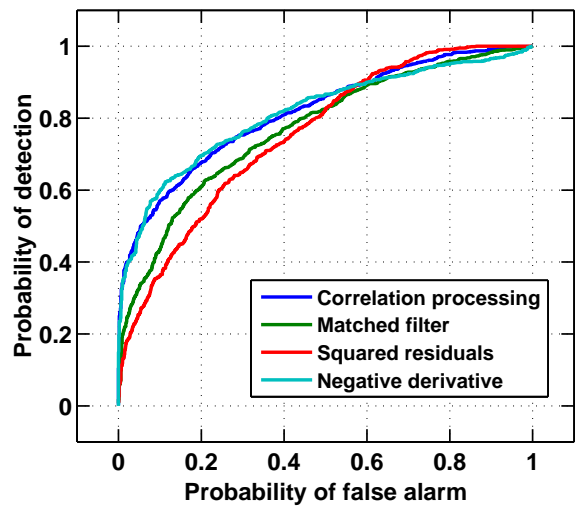

(b)

Figure 4.8: (a) Four ROC curves for a Monte Carlo simulation using surface parameters of $h=43 \mu \mathrm{m}, l=480 \mu \mathrm{m}$. The curves corresponding to correlation processing, matched filter, squared residual and negative derivative-based decisions are in blue, green, red, and cyan, respectively. Only the first lactose peak at $0.53 \mathrm{THz}$ is used, with SNR set at $30 \mathrm{~dB}$. (b) Similar ROC curves, using surface parameters of $h=135 \mu \mathrm{m}, l=420 \mu \mathrm{m}$, the equivalent surface parameters for 40 grit sandpaper, no additive noise.

As seen in Figure 4.8(a), the matched filter significantly outperforms the other approaches in the case where the additive white Gaussian noise is the dominant source of error. However, in the second simulation, the dominant source of error is target variation, as the reflected spectrum varies depending on random surface fluctuations. In this case, the correlation processing and negative derivative approach perform the best. The decreased performance of the matched filter 
detection method in the latter simulation shows that it is not a robust solution in all cases. In fact, any method that depends on exact spectral reproducibility will not perform optimally when target signature varies from sample to sample due to surface roughness.

\subsubsection{Case \#2: C-4 versus (target) lactose}

In the previous section, detection of lactose was performed in the presence of a spectrally flat confounding material, namely HDPE. This was done to emulate the measured data that were analyzed in Section 4.4. However, a Kirchhoff Monte Carlo simulation provides the ability to compute predicted detection performance for common explosive compounds such as C-4. In this section, a numerical simulation comparing C-4 and lactose was conducted. The primary explosive agent, RDX, comprises $91 \%$ of $\mathrm{C}-4$, which is a common plastic explosive used in improvised explosive devices [81]. Figure 4.9 shows amplitude and phase for the reflection coefficient at normal incidence. This study analyzes the quantitative performance of discriminating between C-4 and lactose. Both lactose and C-4 have resonance peaks in the $\mathrm{THz}$ range, which could negate some of the detection performance that was demonstrated in the previous section.

To perform the ROC study, 1,000 numerically surfaces were generated for both C-4 and lactose between 0.1 and $1.2 \mathrm{THz}$. Figure 4.10 shows four ROC curves for a Monte Carlo simulation using surface parameters of $h=43 \mu \mathrm{m}$, 


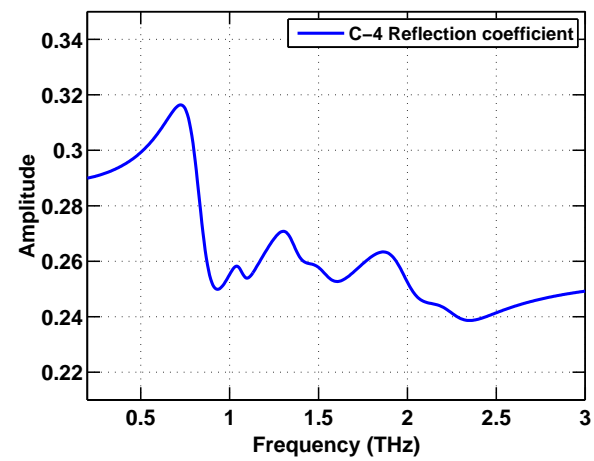

(a)

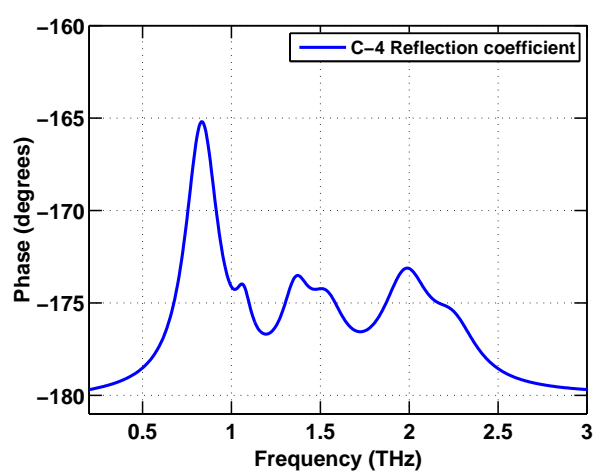

(b)

Figure 4.9: (a) Amplitude of the reflection coefficient of C-4 at normal incidence. Material properties taken from [81]. (b) Phase of the reflection coefficient of C-4 at normal incidence.

$l_{c}=480 \mu \mathrm{m}$. The curves corresponding to correlation processing, matched filter, squared residual and negative derivative-based decisions are in blue, green, red, and cyan, respectively. The first set of curves in Figure 4.10(a) contains SNR of $40 \mathrm{~dB}$, while Figure 4.10(b) has SNR of $30 \mathrm{~dB}$. One observes that the squared residual and the correlation processing approaches outperform other methods. However, when compared to each other, it becomes more complicated to declare a winner, particularly in Figure 4.10(a). Correlation processing outperforms all methods with low probability of false alarm $\left(P_{f a}<0.05\right)$, while the squared residual approach performs better at higher false alarm rates $\left(P_{f a}>0.05\right)$. 


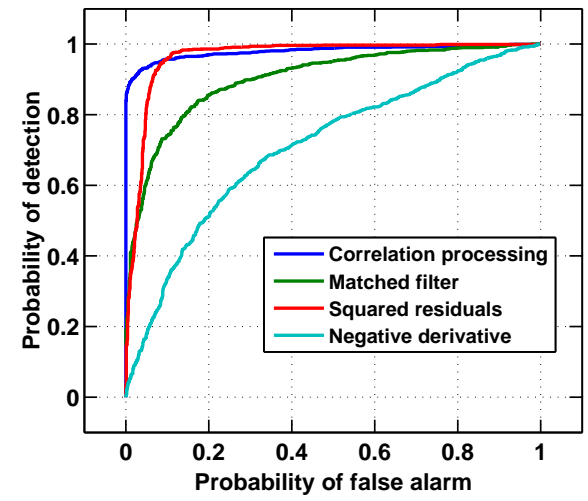

(a)

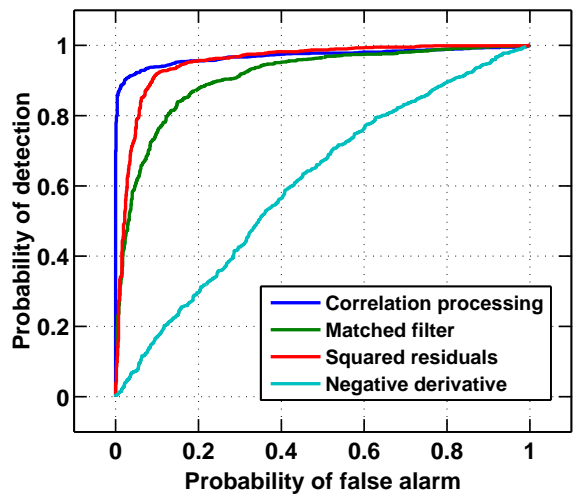

(b)

Figure 4.10: (a) ROC curves for a Monte Carlo simulation using surface parameters of $h=43 \mu \mathrm{m}, l_{c}=480 \mu \mathrm{m}$, and SNR $=40 \mathrm{~dB}$. The curves corresponding to correlation processing, matched filter, squared residual and negative derivativebased decisions are in blue, green, red, and cyan, respectively. (b) Similar ROC curves with SNR of $30 \mathrm{~dB}$. 


\section{Chapter 5}

\section{Synthetic-Aperture Processing}

\subsection{Theoretical imaging formulation}

This section outlines a SA image processing algorithm that was adapted from synthetic-aperture radar. The two most common types of airborne syntheticaperture radar modalities are stripmap mode and spotlight mode. Stripmap mode, considered the conventional approach to SAR, uses a wide beam that illuminates multiple objects at once, while spotlight mode uses a narrow swept beam [82]. Here, only stripmap mode is considered as the beam does not need to be steered to different scattering targets, and is more applicable using a $\mathrm{THz}$ source. First, time-domain delay-and-sum SA imaging formulations are presented, followed by narrowband and broadband frequency-domain SA imaging algorithms. The section concludes with imaging results and characterization of imaging performance.

One of the key advantages of SA image processing is high resolution potential. While optical imaging systems have a lateral imaging resolution that is inversely proportional to beamwidth, the opposite is true for phased-array techniques, such as SA imaging [13]. For a synthetic (or phased) array, the lateral imaging 
resolution is inversely proportional to beamwidth. From basic antenna theory and Fourier optics, a larger antenna array results in better image resolution. According to [13], the lateral resolution in traditional synthetic-aperture radar, $\rho$, is given by

$$
\rho=\frac{\lambda R}{2 L_{\text {array }}},
$$

where $\lambda$ is the free space wavelength of radiation and $R$ is the distance from the array to the target. The variable, $L_{\text {array }}$, is the length of the synthetic array, which is proportional to the transmitting and receiving antenna beamwidth. That is, the wider the beam, the more elements can be coherently added together and the better the lateral resolution becomes.

As mentioned earlier, a simple implementation of $\mathrm{THz} \mathrm{SA}$ imaging can be done purely in the time domain with a delay-and-sum approach. In order to synthesize a 3-D stack of images from an $M \times N$ synthetic array, an image plane is defined as a grid of pixels lying on a plane at some focusing depth, $z_{l}$. A one-dimensional example of time-domain delay-and-sum beamforming is shown in Figure 5.1. The vector describing the location of each image pixel is given by $\vec{r}_{h j l}$, which has coordinates $\left[\begin{array}{lll}x_{h} & y_{j} & z_{l}\end{array}\right]$, where $h$ is the pixel row index, $j$ is the pixel column index, $l$ is the vertical stack index, and $z_{l}$ is the focusing depth below the source. The vector, $\vec{R}_{m n}$, has elements $\left[\begin{array}{lll}x_{m} & y_{n} & 0\end{array}\right]$, for the $x_{m}$ and $y_{n}$ 

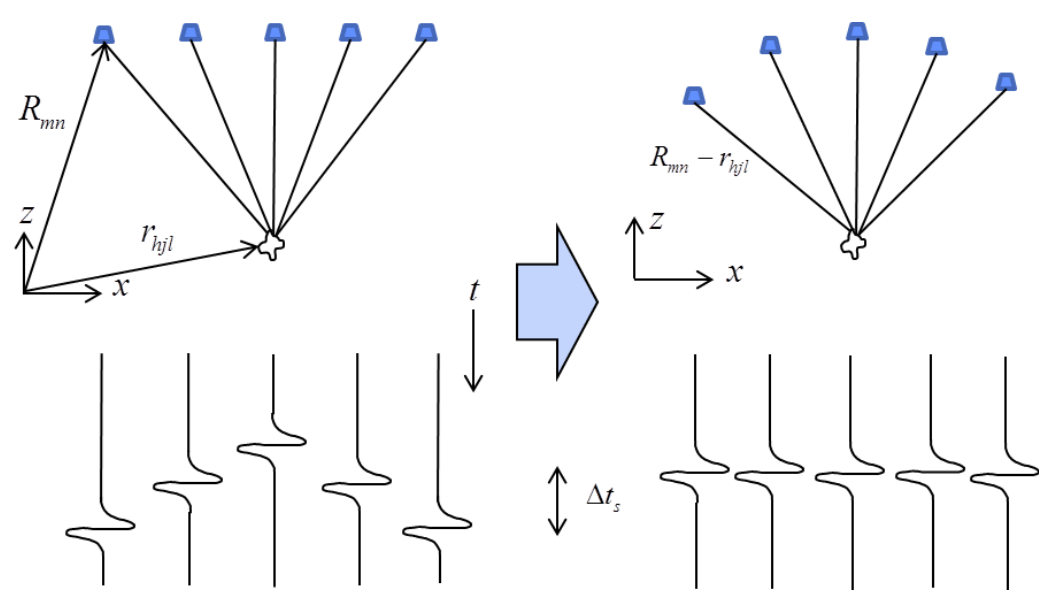

Figure 5.1: (above) One-dimensional linear array being used in a syntheticaperture configuration. The path lengths connecting the synthetic array to the desired imaging target before and after correction, revealing how beamforming reshapes array into a circle. (below) The time-domain waveforms corresponding to each sensor in the array before and after delay.

sensor positions. All sensor positions are assumed to lie on the $x y$ plane. The received electric field at each sensor position is described by $E\left(x_{m}, y_{n}, t\right)$. The time-domain focused field, $C_{T D}\left(\vec{r}_{h j l}\right)$, for any given desired image pixel, $\vec{r}_{h j l}$, is given by

$$
C_{T D}\left(\vec{r}_{h j l}\right)=\sum_{m=1}^{M} \sum_{n=1}^{N} E\left(x_{m}, y_{n}, t+t_{s}\right),
$$

where the time shift, $t_{s}$, is given by

$$
t_{s}=\frac{\left|\vec{R}_{m n}-\vec{r}_{h j l}\right|}{c}
$$

and $c$ is the speed of light in a vacuum. Each pulse is coherently added after 
an appropriate time shift, which accounts for the path length between each sensor position and desired imaging pixel [83]. This is similar to traditional SAR processing, which often uses a relatively narrowband linear frequency modulated chirp as a waveform.

\subsubsection{Frequency-domain synthetic-aperture processing using a virtual source}

There are multiple reasons to conduct the synthetic-aperture processing in the frequency domain instead of the time domain. First and foremost, spectroscopic information must be extracted from the frequency domain. In addition, the transmitted $\mathrm{THz}$ pulses potentially contain frequency-dependent distortion. Synthetic-aperture radar (SAR) data can be processed in the time domain, as these systems typically use linear frequency modulated chirps that are relatively narrowband. Radar chirps may only have a bandwidth of an octave (i.e., $5 \mathrm{GHz}$ to $10 \mathrm{GHz}$ ), while $\mathrm{THz}$ pulses can have bandwidths of well over an decade (0.1 $3 \mathrm{THz}$ ). The distortion of such broadband $\mathrm{THz}$ pulses can vary across the transmitted beam pattern. Therefore, it is beneficial to conduct SA image processing in the frequency domain to prevent addition of incoherent pulses. This claim is substantiated in the following section.

A wide beamwidth is desired for SA imaging, as it allows more sensors to be 


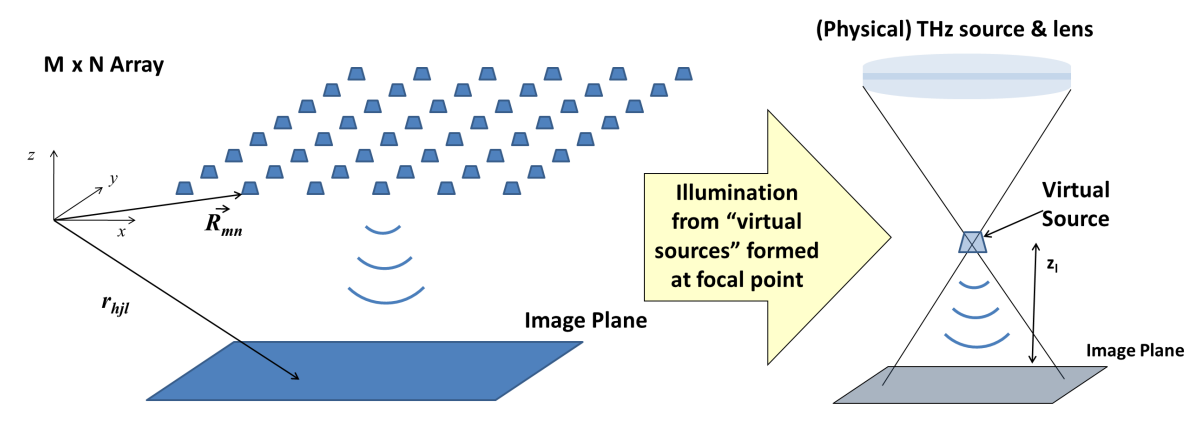

Figure 5.2: 2-D planar array used in a synthetic-aperture configuration. Virtual sources are created by lowering the desired object below the physical focal point of the one-inch lens. The image vector, $\vec{r}_{h j l}$, connects the origin to the desired image pixel. $\vec{R}_{m n}$ describes the distance to the $x_{m}$ and $y_{n}$ virtual source positions. The focusing depth, $z_{l}$, is the vertical distance between the sensor position and the imaging plane.

coherently added. This diverging beam was achieved by using a one-inch focusing lens to create a virtual point source at the focal point of the lens [83]. The object to be imaged was intentionally placed below the focal point, allowing for traditional SA techniques to be used. The T-Ray 4000 monostatic transceiver with the afixed one-inch lens was then raster scanned in two directions to create a $M \times N$ element planar synthetic array, as shown in Figure 5.2. As with (5.2), a 3-D stack of images can be generated from a 2-D $M \times N$ synthetic array. The geometric parameters, such as $\vec{r}_{h j l}$, required for frequency-domain processing are the same as those used in the previous section. The received electric field at the $x_{m^{t h}}$ and $y_{n^{t h}}$ virtual source position must be transformed into the frequency domain via a discrete Fourier transform, which becomes $E\left(x_{m}, y_{n}, f_{q}\right)$. The narrow-band focused field, $C_{N B}\left(\vec{r}_{h j l}, f_{q}\right)$, for any given desired image pixel, $\vec{r}_{h j l}$, is given by 


$$
\begin{gathered}
C_{N B}\left(\vec{r}_{h j l}, f_{q}\right)=\sum_{m=1}^{M} \sum_{n=1}^{N} F_{m n}\left(\vec{r}_{h j l}\right) \\
\times E\left(x_{m}, y_{n}, f_{q}\right) e^{i 2 k_{0}\left|\vec{R}_{m n}-\vec{r}_{h j l}\right|},
\end{gathered}
$$

with the two dimensional Gaussian tapering window, $F_{m n}\left(\vec{r}_{h j l}\right)$, with width, $\sigma$, defined as

$$
F_{m n}\left(\vec{r}_{h j l}\right)=\frac{1}{2 \pi \sigma^{2}} e^{\frac{-\left|x_{h}-x_{n}\right|^{2}-\left|y_{j}-y_{m}\right|^{2}}{2 \sigma^{2}}},
$$

with the total sum of the tapering window normalized so that

$$
\sum_{m=1}^{M} \sum_{n=1}^{N} F_{m n}\left(\vec{r}_{h j l}\right)=1 .
$$

The tapering window is chosen to be a Gaussian to reduce side lobe artifacts. All sensor contributions are coherently added together after appropriate phase shifts which depend on the roundtrip path length between each sensor position and desired imaging pixel, and the free space wavenumber, $k_{0}$. The essential difference between time-domain processing in (5.2) and frequency-domain processing is that the time shift is replaced by a linear phase shift across frequency applied to all combinations of sensor elements and desired image pixels [83]. This basic SA imaging algorithm works quite well for simple imaging configurations. However, 
in order to create accurate images of buried or hidden objects, propagation effects such as refraction at a layer boundary should be included (3-D imaging results using refraction are shown in Section 5.3.2). In addition, narrowband SA imaging does not utilize the full bandwidth inherent in the THz pulse, resulting poor depth resolution. However, broadband averaging can be used to fix this problem and achieve greatly enhanced depth resolution.

\subsubsection{Broadband 3-D synthetic-aperture processing}

Coherently averaging the complex synthesized field across frequency can dramatically improve the depth of focus due to constructive interference. The broadband averaged intensity, $C_{B B}\left(\vec{r}_{h j l}\right)$, is given by

$$
C_{B B}\left(\vec{r}_{h j l}\right)=\left|\frac{1}{Q} \sum_{q=1}^{Q} C_{N B}\left(\vec{r}_{h j l}, f_{1}+q \Delta f\right)\right|^{2},
$$

where $f_{1}$ is the starting frequency in the band. The parameter $Q$ is the number of frequencies contained in the band and $\Delta f$ is the frequency resolution. It is easy to see the motivation for frequency averaging. Considering that each sensor records a $\mathrm{THz}$ time-domain waveform, the electric field at each frequency bin is complex, meaning it contains both amplitude and phase information. After the summation in (5.7), there will be only one depth where all $C_{N B}$ values add coherently. Therefore, one can achieve constructive interference only at the focal 
depth, and all other depths will interfere destructively. One can think of the summation in (5.7) as an inverse Fourier transform at $t=0$, normalized by Q. Taking a broadband average will increase SNR, as the noise is uncorrelated across frequency. Another advantage is that imaging artifacts such as side lobes are frequency dependent and are mitigated by using a broadband average. Later sections in the chapter characterize the enhanced performance using a broadband average.

\subsection{Additional processing considerations}

This section outlines some of the preprocessing routines that are conducted before the implementing the core SA imaging algorithm described in the previous section. The first step combines the prewhitening of the received electric field and a phase correction. The other significant process is background subtraction, which removes clutter in the time-domain waveform recorded from the T-Ray 4000 system. There is an additional step that accounts for virtual source drifts as a function of frequency. However, this is not used in the body of this dissertation, and is described in Appendix E. 


\subsubsection{THz beam distortion}

To substantiate the claim that $\mathrm{THz}$ pulses are subject to distortion, a small steel ball bearing, $1 \mathrm{~mm}$ in radius, was measured at multiple look angles relative to the center axis of the transmitted beam below the virtual source. The backscattering coefficient from a ball bearing does not depend on viewing geometry due to its spherical symmetry. Therefore, imaging a small ball bearing gives a reasonable approximation of the beampattern, which is the complex electric field measured as a function of look angle and frequency relative to the source. Horizontal cuts of magnitude and unwrapped phase of the beam pattern are plotted as a function of horizontal displacement in Figure 5.3(a) for $0.5 \mathrm{THz}$. For example, the source is directly over the center of the ball bearing at zero degrees, or when the horizontal displacement is zero. The phase shows a hyperbolic shape with respect to horizontal displacement, as one would expect. However, the magnitude is quite erratic, and varies greatly with frequency and angle. At any given angle, the frequency dependence of the magnitude translates to distortion in the time domain. However, the phase is intact across all angles, which is the key element in frequency-domain SA imaging.

Another way to interpret $\mathrm{THz}$ pulse dispersion is by viewing two different waveforms at different viewing angles, which should be comparable in pulse width. Figure 5.3(b) shows the electric field time-domain waveform for $8^{\circ}$ and $16^{\circ}$ relative 


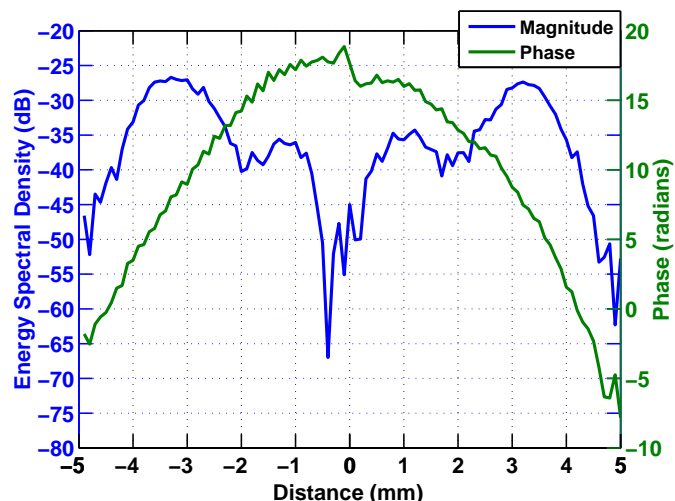

(a)

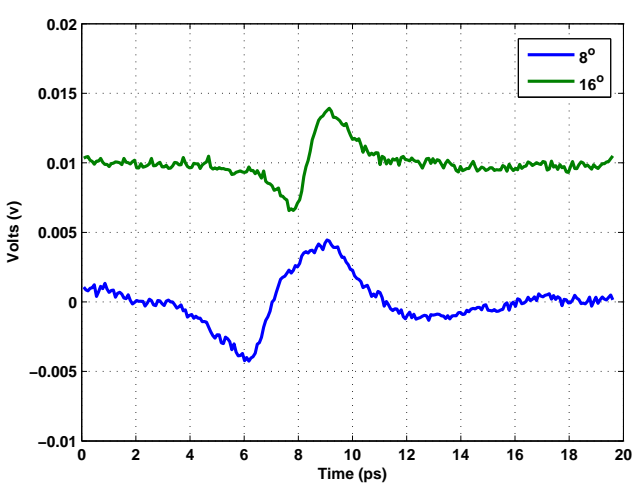

(b)

Figure 5.3: (a) Magnitude and unwrapped phase recorded for a monostatic source as a function of lateral distance from a ball bearing, with a constant vertical height of $1 \mathrm{~cm}$, at $0.5 \mathrm{THz}$. The magnitude shows irregular behavior, while the unwrapped phase shows a hyperbolic shape that is robust for SA imaging. (b) Two THz pulses reflected from a steel ball bearing with $1 \mathrm{~mm}$ radius at $8^{\circ}$ and $16^{\circ}$, relative to the main axis of the transmitted beam.

to the axis of the main beam. There is a time shift due to the different path length between source and the ball bearing for both angles. However, both pulses are visibly different and have varying pulse widths of 3 and 5 ps, respectively, due to distortion.

In order to mitigate the irregular beampatterns present in the source, a normalization is applied before beamforming. This normalization is sometimes described as prewhitening. Before all beamforming, the electric field, $E\left(x_{m}, y_{n}, f_{q}\right)$, is phase shifted and normalized according to

$$
E\left(x_{m}, y_{n}, f_{q}\right)=\frac{e^{-i 2 \pi f\left(t_{g}-t_{v s}\right)} \mathscr{F}\left\{E\left(x_{m}, y_{n}, t\right)\right\}}{\left|\mathscr{F}\left\{E\left(x_{m}, y_{n}, t\right)\right\}\right|+W_{0}},
$$


where $\mathscr{F}$ denotes a discrete Fourier transform, as shown in (3.1). The timedomain waveform is gated around the received pulse. Equation (5.8) suppresses magnitude information, while preserving the more meaningful phase information. However, an offset, $W_{0}$, is used in the denominator to avoid dividing by zero at frequencies where there is little transmitted power. $W_{0}$ is tied to the average standard deviation of $\mathscr{F}\left\{E\left(x_{m}, y_{n}, t\right)\right\} \mid$. Because of the normalization in (5.8), $C_{B B}$ becomes unitless. However, the values of $C_{B B}$ are referred to as SA normalized intensity, which has a maximum value of unity.

The exponential term in (5.8), $e^{-i 2 \pi f\left(t_{g}-t_{v s}\right)}$, accounts for the linear phase accumulation introduced by time-gating the $\mathrm{THz}$ pulse. The frequency-domain SA focusing technique assumes that phase begins accumulating at the source position (i.e., if a target were placed just below the source, there would be zero phase across all frequency). This would imply that one should begin the time-gate at the location of the virtual source. To improve SNR, however, it is preferable to time-gate only the received signals, beginning at time $t_{g}$. If the data started at time $t_{v s}$, corresponding to the location of the virtual source, there would be no need for any correction. Therefore, the linear phase correction must be applied in (5.8) that accounts for the time difference between the virtual source and the beginning of the time-gate, $t_{g}-t_{v s}$. 


\subsubsection{Mitigation of system-level etalon effects}

Another important preprocessing step is the mitigation or removal of systemlevel etalon effects. These can be described as simply additive scaled THz pulses that are inherent in all waveforms recorded with the collinear (monostatic) head. As mentioned in Chapter 3, the $\mathrm{THz}$ pulse is generated by focusing a femptosecond laser on a photoconductive antenna. The THz pulse then propagates through a beam splitter to a polyethylene focusing lens. The beamsplitter diverts the reflected THz wave to the receiving antenna. However, there are multiple reflections from within the polyethylene lens, beamsplitter, and other elements, that are detected by the receiver, and are recorded in the time-domain waveform. These extra reflections add coherently to THz pulse reflected from the sample, and can corrupt the desired $\mathrm{THz}$ waveform.

As an example, Figure 5.4 shows two waveforms. The 'background', shown in green, is recorded with nothing placed in the path of the THz beam. The curve shown in blue corresponds to a waveform recorded with a sample in the path of the THz beam, in this case, a polyethylene pellet containing lactose. This phenomenon does not occur in transmission mode, as all reflections from within the lens occur after the incident $\mathrm{THz}$ pulse, and thus can be time-gated out. These reflections arrive concurrently with the desired THz pulse.

In order to remove these pulses, they must be subtracted from any recorded 


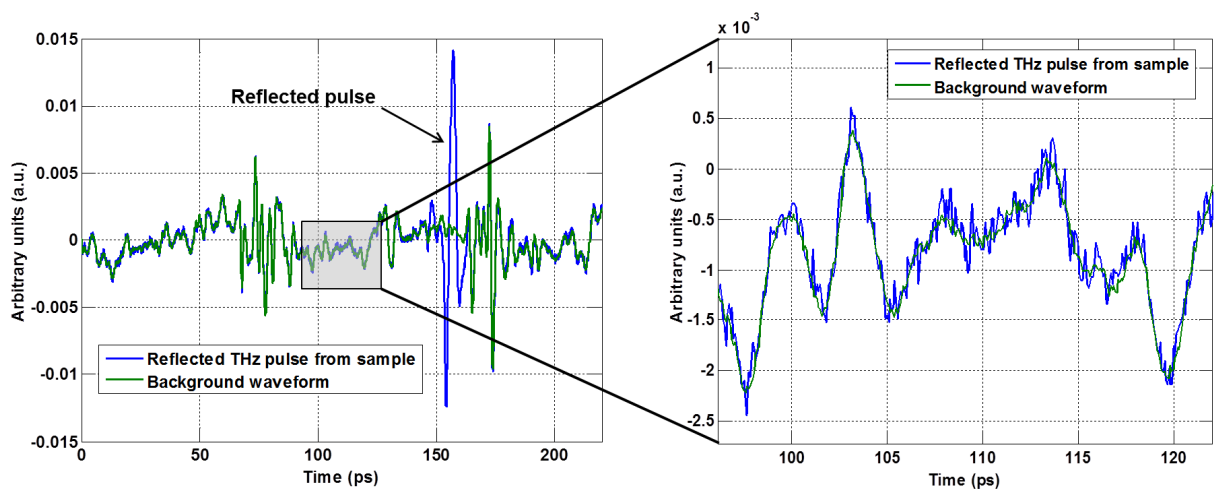

Figure 5.4: (left) Sample waveform (blue) and background waveform (shown in green) taken with nothing in the path of the $\mathrm{THz}$ beam. (right) Zoomed-in section showing both sample and background waveforms.

sample waveform. However, it is not practical to record a background waveform for every sample waveform, especially in an imaging environment. When imaging, it is desirable to take a single background before and after the image. One key issue when subtracting background from sample waveform becomes whether or not the two are in-phase. That is, are both waveforms exactly time-aligned. Any drift between background and sample will negate the entire processing step. Because system drift is a known problem, it cannot be assumed that the background reflections are always time-aligned with the sample.

Therefore before subtracting the background from each sample waveform, a cross correlation is used to time-align each signal. A similar method is outlined in [84]. A section of the background waveform, denoted as, $E_{\text {back }}(t)$ is cross-correlated with a corresponding section of the sample waveform, denoted, $E_{\text {sample }}(t)$. This cross correlation, denoted $R_{b s}(t)$, can be written as 


$$
R_{b s}(t)=\int_{-\infty}^{\infty} E_{b a c k}^{*}(\tau) E_{\text {sample }}(t+\tau) d \tau .
$$

The time delay, $t_{\max }$, can be found by taking the argument of the maximum of the cross correlation between $R_{b s}(t)$. This can be written as

$$
t_{\max }=\underset{t}{\arg \max } R_{b s}(t) .
$$

The background waveform was then shifted by $t_{\max }$ so that both are timed aligned. The corrected waveform can be written as

$$
E_{\text {sample }}(t)-E_{\text {back }}\left(t-t_{\max }\right) .
$$

\subsection{3-D broadband $\mathrm{SA} \mathrm{THz}$ imaging results}

This section provides experimental evidence that 3-D broadband THz imaging holds great potential for non-destructive evaluation, such as surface profiling, hidden object detection with sub-wavelength depth resolution. In order to demonstrate these capabilities, various objects such as coins and electronic cables were imaged in the broadband SA configuration described in Section 5.1. 


\subsubsection{Surface profiling with metallic interface}

To provide an example of surface profiling, using $\mathrm{THz} \mathrm{SA} 3-\mathrm{D}$ imaging, a metallic coin was used. An American dime was chosen to demonstrate the depth resolution capability due to it's subtle surface features. A dime, $17.9 \mathrm{~mm}$ in diameter and $1.35 \mathrm{~mm}$ in thickness, contains multiple curved facets on a single metallic interface, which should provide strong returns. The dime was placed $1 \mathrm{~cm}$ below the virtual source of the SA imaging system. The data were recorded in a 2 -D synthetic array $18 \mathrm{~mm} \times 18 \mathrm{~mm}$, centered over the dime. This resulted in a $180 \times 180$ data set with $100 \mu \mathrm{m}$ spacing.

Figure 5.5(a) shows an optical image of a dime, while Figure 5.5(b) shows the $\mathrm{THz}$ SA image of the dime using a broadband average from 0.7-1.1 $\mathrm{THz}$ from (5.7). A stack of images was computed for different focusing depths and an average was taken across all said depths. The color scale on Figure 5.5(b) is SA normalized intensity, which has a maximum value of unity due to the normalization in (5.8). The maximum value chosen for the color bar was $2 \times 10^{-7}$. The SA normalized intensities were small due to limited diffuse scattering on portions of the dime and reduced SNR higher in the frequency range. One important observation from Figure 5.5(b) is that SA imaging primarily relies on scattering at the edges and facets present on the surface of the dime. This illustrates two distinct advantages of this imaging modality; there is less sensitivity to alignment and 


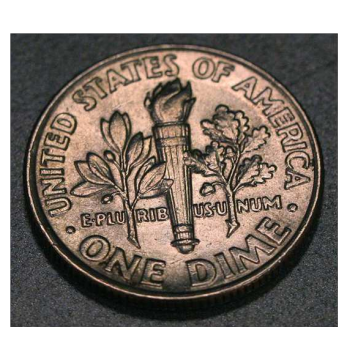

(a)

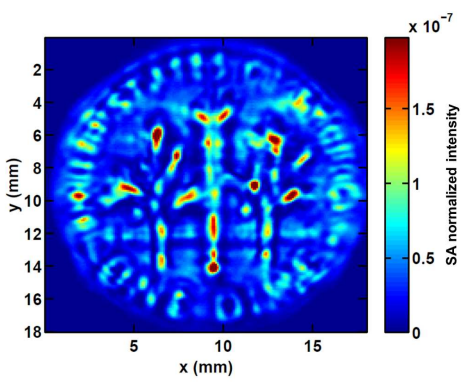

(b)

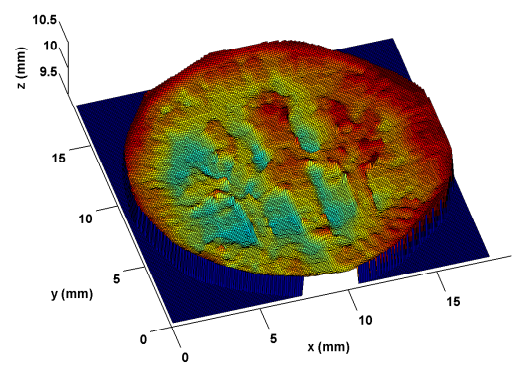

(c)

Figure 5.5: (a) Optical image of an American dime, $17.9 \mathrm{~mm}$ in diameter. The dime, primarily made with copper, provides an example of a highly reflective metallic interface for $\mathrm{THz}$ surface profiling. (b) Broadband SA image of the dime using a $18 \mathrm{~mm} \times 18 \mathrm{~mm}$ synthetic array. A frequency range of 0.7-1.1 THz was used, and the image was averaged across depth. (c) Synthetic-aperture surface profile the dime using a broadband average of 0.3-1.1 THz. Profile was created by taking the depth of maximum SA Normalized Intensity at each pixel location.

smooth surfaces facing the array do not appear especially bright and therefore do not wash out the rest of the image. Just as with side-looking radar, syntheticaperture imaging is less sensitive to specular reflections from smooth interfaces because specular returns have narrow beamwidths, thus limiting the number of sensors that can be coherently added [68].

A surface profile of the dime was computed by finding the depth corresponding 


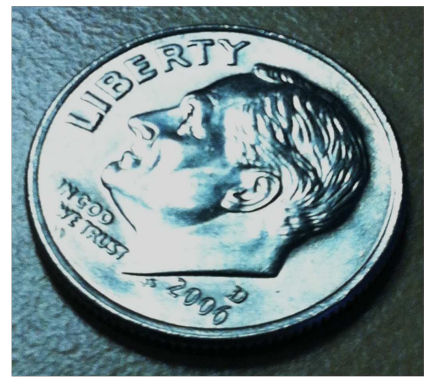

(a)

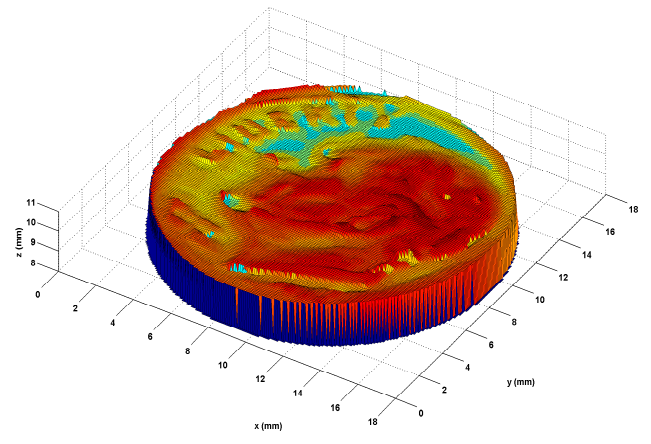

(b)

Figure 5.6: (a) American dime. (b) THz profile averaged from 0.25-1.0 THz.

to the maximum SA normalized intensity for every pixel. The frequency range for the surface profile was chosen to be $0.3-1.2 \mathrm{THz}$. In order to be shown in the surface profile, the maximum value for each pixel must exceed a threshold equal to 0.5 times the standard deviation of the image. The profile was then smoothed and is shown in Figure 5.5(c). The maximum vertical relief from the reverse of the dime was measured with a micrometer to be $130 \mu \mathrm{m}$, roughly half of $250 \mu \mathrm{m}$, the shortest wavelength included in band. A potential application for SA imaging would be to create surface profiles of hidden layers without physically tampering with the object. Product defects hidden within packaging layers could then be detected and imaged with this modality.

Similarly, the front side of the dime showing Franklin D. Roosevelt was imaged and profiled in similar manor. Figure 5.6(a) shows an optical image of a dime, while Figure 5.6(b) shows a surface profile of the dime. The search space in the 


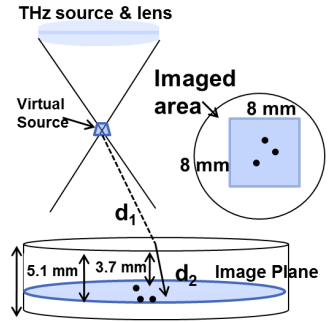

(a)

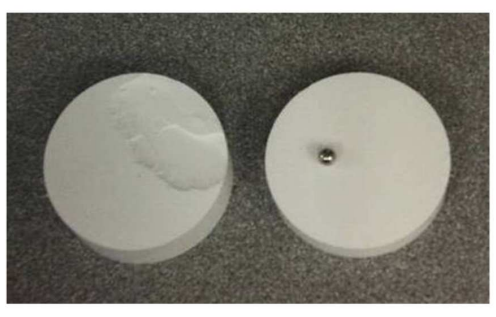

(b)

Figure 5.7: (a) Pellet made of high-density polyethylene with three embedded ball bearings $0.5 \mathrm{~mm}$ in radius. Sample was $1 \mathrm{~cm}$ below virtual source and imaged in a SA configuration. (b) A similar sample of a pellet was split apart, showing the ball bearing embedded inside.

$z$-direction was increased on the top portion of the profile to capture the full extent of the lettering. The surface outside the edge of the dime was suppressed for clarity. The frequency range for this surface profile was chosen to be $0.25-1.0$ $\mathrm{THz}$

\subsubsection{3-D Target depth localization}

In order to demonstrate and test the depth isolation capability of the SA focusing technique, three steel ball bearings were embedded in a pellet consisting of HDPE, as shown in Figure 5.7(a). A similar sample was split apart and shown in Figure 5.7(b). HDPE is optically opaque; however it is transparent to $\mathrm{THz}$ radiation and is a powder that can be pressed with inclusions in known locations. In this section, SA imaging was used to localize the ball bearings embedded in the HDPE pellet. 
To construct the sample, 5 grams of HDPE was compressed into a pellet using a hydraulic press, $4 \mathrm{~cm}$ in diameter. The pellet was subjected to five tons of force for one minute. Next, a single steel ball bearing ( $0.5 \mathrm{~mm}$ radius) was placed on top of this 5 gram-pellet along with 1.5 more grams of HDPE. This was inserted into the press to create one pellet that contains a total of 6.5 grams of HDPE and one ball bearing. Then the process was repeated, placing two more ball bearings on top of the existing pellet along with 5 more grams of HDPE. The final sample was flipped so that the layer including a single ball bearing was on top.

The final pellet contained 11.5 grams of HDPE and three ball bearings separated at different depths and different horizontal locations. The time-of arrival of the terahertz pulse through the HDPE, revealed the location of the first ball bearing to be $3.7 \mathrm{~mm}$ below the surface. Using this same technique, the depth of the second two ball bearings was found to be approximately $5.1 \mathrm{~mm}$. The top of the HDPE pellet was lowered $1 \mathrm{~cm}$ below virtual source, which coincides with the focal point of the one-inch focusing lens attached to the T-Ray $400 \mathrm{THz}$ system. An $80 \times 80$ data set was recorded with $100 \mu \mathrm{m}$ spacing.

In order to reconstruct layers of the embedded structure, a modified version of (5.7) was used. More details on the modified version are given in Appendix F. Figures 5.8(a) and 5.8(b) show the image reconstructed at depths of $3.7 \mathrm{~mm}$ and $5.1 \mathrm{~mm}$. Both images used a broadband average of 0.3-0.4 THz, with a maximum 


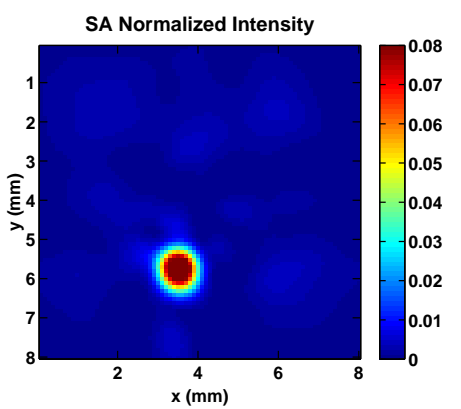

(a)

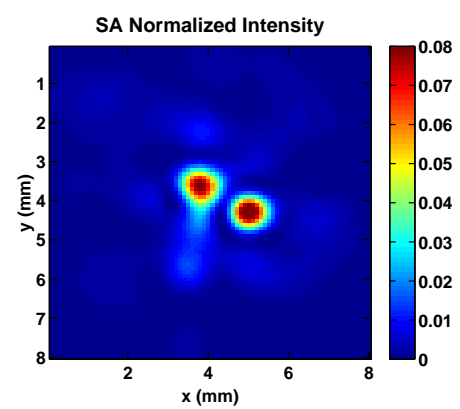

(b)

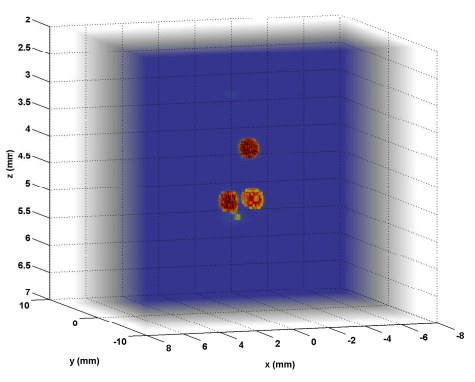

(c)

Figure 5.8: (a) SA image of a ball bearing (0.5 $\mathrm{mm}$ radius) embedded in highdensity polyethylene. Focusing depth is $3.7 \mathrm{~mm}$ below the surface of the sample. (b) SA image of ball bearings ( $0.5 \mathrm{~mm}$ radius). Focusing depth is $5.1 \mathrm{~mm}$ below the surface of the sample. The two ball bearings are separable in depth. (c) A thresholded, 3-D, broadband image of two ball bearings.

value for SA normalized intensity of 0.08. These figures clearly demonstrate that two sets of ball bearings are resolvable in depth. Therefore, a bandwidth of 0.1 $\mathrm{THz}$ is able to resolve objects separated by $1.4 \mathrm{~mm}$ in depth. Figure 5.8(c) shows a 3-D $\mathrm{THz}$ image of the ball bearings using a bandwidth of $0.3-0.5 \mathrm{THz}$. A threshold was used in the 3-D image to remove noisy voxels. 


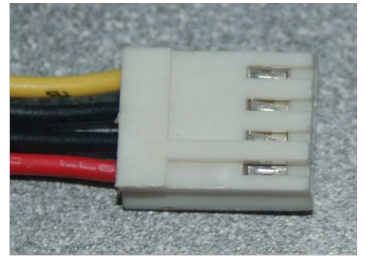

(a)

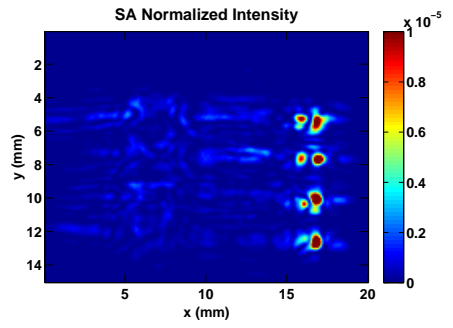

(c)

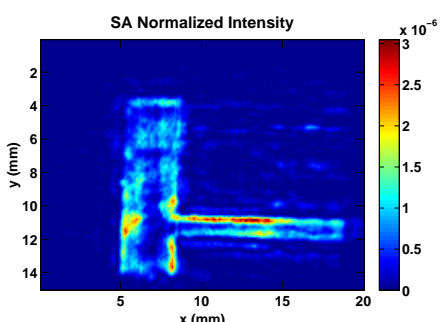

(b)

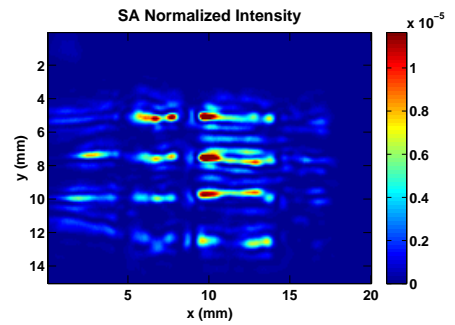

(d)

Figure 5.9: (a) Optical image of a floppy drive power connector, with four metal traces connected with insulated wires. (b) SA image of connector. Focusing depth is $10.06 \mathrm{~mm}$ at the top of the casing. (c) SA image of connector, focused at $11.82 \mathrm{~mm}$. This corresponds to the location of the rounded metal connector on the end of connector. (d) SA image of connector focused at $12.39 \mathrm{~mm}$. Four hidden metal traces that are not seen in the optical image appear bright due to high reflectivity and sharp edges.

\subsubsection{Volumetric imaging of 3-D dielectric structures}

To further serve as an example of the beam-forming capability at various depths, a four-pronged floppy drive power connector was imaged in a SA imaging configuration. The plug dimensions were $11 \mathrm{~mm}$ wide by $14 \mathrm{~mm}$ long. Also present in the image are four insulated wires feeding the plug. An optical image of the cable plug is shown in Figure 5.9(a). 


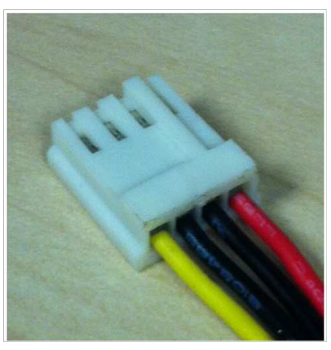

(a)

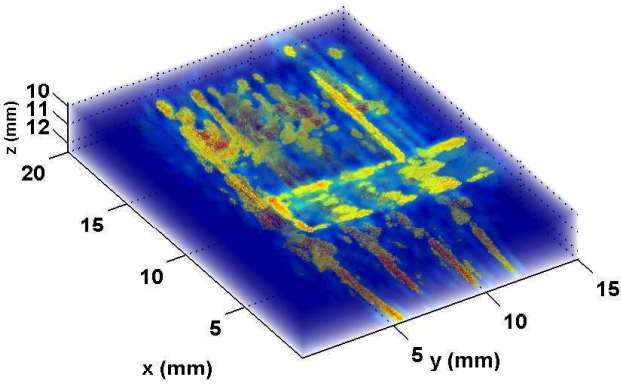

(b)

Figure 5.10: (a) An optical photo of a floppy drive power connector used in electronics. (b) 3-D THz image of connector averaged from 0.3-1.2 THz.

The casing material is unknown. However, floppy drive power connector casings are typically nylon. The connector was lowered so that the top of the casing was $1 \mathrm{~cm}$ below the virtual source. The virtual source was scanned to form a $200 \times 150$ array, with an element spacing of $100 \mu \mathrm{m}$. Figures 5.9(b) - 5.9(d) show three SA images of the ribbon plug at three different focusing depths, 10.06, 11.82 $\mathrm{mm}$, and $12.39 \mathrm{~mm}$, respectively. The images were computed using (5.7) assuming free space propagation between target and sensor position. The metal layers are buried within inhomogeneous dielectric layers, preventing the use of a more complicated imaging kernel. However, iterative techniques could be used to find the exact structure of the cable. The frequency range was chosen to be $0.4-1.2$ THz.

The 3-D THz image was computed using a bandwidth of $0.3-1.2 \mathrm{THz}$, and is shown in Figure 5.10(b) using a logarithmic scale. For comparison, an optical 
photo is shown at the same angle as the 3-D THz image in Figure 5.10(a). Just as before, a threshold was used in the 3-D image to remove noisy voxels. The buried metal traces are visible in Figure 5.10(b), though the view is obscured on the termination end of the plug by the dielectric casing structure. For example, the notch in the top of the casing can be seen in the $3-\mathrm{D} \mathrm{THz}$ image. It is important to note that the object does not need to be metallic to provide strong enough returns for a 3-D image, as evident by the detailed casing structure in the image.

\subsubsection{HDPE pellet with multiple embedded scatterers}

An additional pellet was embedded with multiple $500 \mu \mathrm{m}$ radii steel ball bearings. The purpose of this experiment was to investigate shadowing effects of $\mathrm{THz} 3-\mathrm{D}$ imaging. The SA depth focusing method was compared to the more conventional $\mathrm{THz}$ time-domain reflection imaging, which converts time-of-arrival to depth information after an assumption of index of refraction [85]. Figure 5.11 shows imaging configurations of both SA 3-D (left) and conventional THz timegate depth focusing method (right).

To construct the ball bearing, several grams of HDPE were mixed with approximately 500 ball bearings and pressed using a hydraulic press. The backside of the pellet, which reveals some partially exposed ball bearings is shown in Figure 5.11(c). The top side of the pellet was lowered $1 \mathrm{~cm}$ below the virtual source. 


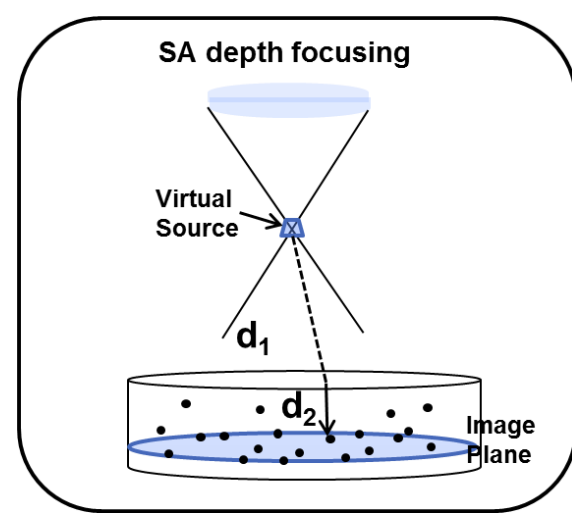

(a)

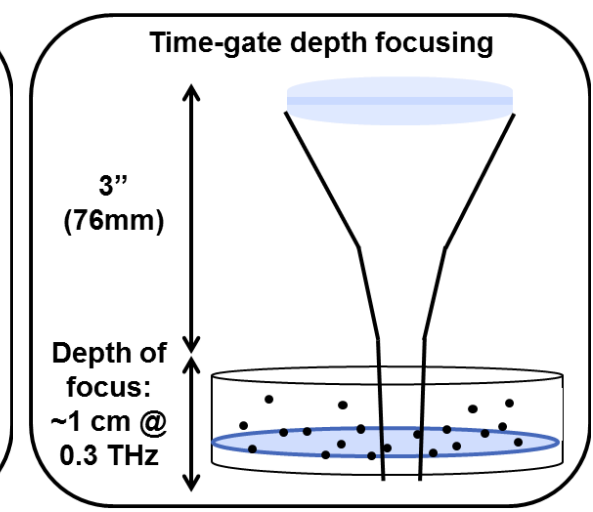

(b)

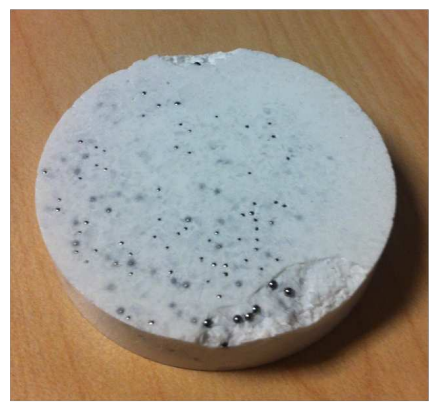

(c)

Figure 5.11: (a) THz SA imaging configuration (b) THz time-gate depth focusing geometry using a 4 ps sliding time window. (c) HDPE pellet embedded with several $500 \mu \mathrm{m}$ radii steel ball bearings.

The transceiver was scanned to create a $200 \times 200$ synthetic array dataset.

Figure 5.12(a) shows the SA THz image of the ball bearings at the same angle using a frequency average spanning 0.3-0.5 THz. Just as with the 3-D connector, a threshold was used in both 3-D images to remove noisy voxels. One can see all the ball bearings appear as separate red blotches.

Figure 5.12(b) shows the conventional $\mathrm{THz} 3-\mathrm{D}$ image of the ball bearings 


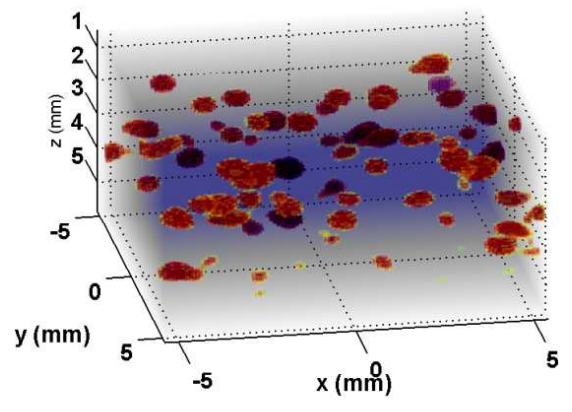

(a)

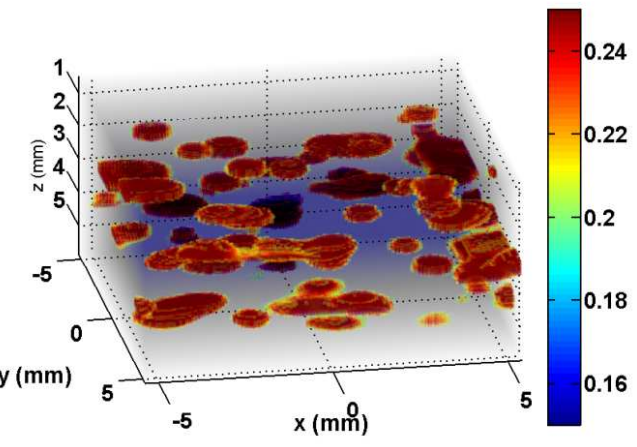

(b)

Figure 5.12: (a) SA image of high-density polyethylene pellet consisting of multiple $500 \mu \mathrm{m}$ radii steel ball bearings. A broadband average spanning $0.3-0.5 \mathrm{THz}$ was used. (b) $\mathrm{THz} 3-\mathrm{D}$ image of the same pellet using a 4 ps time-window for depth focusing.

embedded in HDPE. In this image, a 4 ps sliding time-window was used for depth focusing. The time-window helps to remove $\mathrm{THz}$ pulse artifacts and is necessary for viewing spectral information. The maximum voxel value was set to unity for both images. The colorscale to the right of Figure 5.12(b) applies to both images.

Comparing Figures 5.12(b) and 5.12(a) visually, the SA image is generally cleaner with less blurring. The $\mathrm{THz} \mathrm{SA}$ image also contains fewer missed targets and better lateral and depth resolutions, as defined by the full width at half $\max (\mathrm{FWHM})$ of the image intensity. Although many ball bearings are mostly separable in the conventional image, the lateral resolution appears to be poor, with some targets appearing as large as $1 \mathrm{~mm}$ in diameter. Average lateral and 
depth resolutions are given in Table 5.1, as well as standard deviations of both values from target to target. The SA image has better lateral resolution, with $756.9 \mu \mathrm{m}$ compared to $811 \mu \mathrm{m}$ for conventional imaging.

Table 5.1: Imaging resolution metric comparison between SA depth focusing and time-gate depth focusing.

\begin{tabular}{|c|c|c|}
\hline & SA depth focusing & Time-gate depth focusing \\
\hline Depth resolution (mean) & $442.9 \mu \mathrm{m}$ & $439.2 \mu \mathrm{m}$ \\
\hline Depth resolution (std) & $44.1 \mu \mathrm{m}$ & $57.4 \mu \mathrm{m}$ \\
\hline Lateral resolution (mean) & $756.9 \mu \mathrm{m}$ & $811.9 \mu \mathrm{m}$ \\
\hline Lateral resolution (std) & $182.6 \mu \mathrm{m}$ & $225.3 \mu \mathrm{m}$ \\
\hline
\end{tabular}

In addition to better overall resolution capability, the SA imaging technique leads to considerably more consistent results, with at least $20 \%$ reduction in the standard deviation for both resolutions. This is possibly due to shadowing effects, as the time-gating approach contains a narrow beam and which can be obstructed. Meanwhile, SA imaging techniques take advantage of multiple look angles, and the same obstruction will not to be present at all look angles.

\subsection{Characterization}

In order to produce meaningful system specifications, a preliminary experiment was conducted using a steel ball bearing with a $1 \mathrm{~mm}$ radius. The ball bearing was lowered approximately $1 \mathrm{~cm}$ below the virtual source. The data were recorded with a $100 \times 100$ synthetic array, $100 \mu \mathrm{m}$ spacing. The ball bearing is 


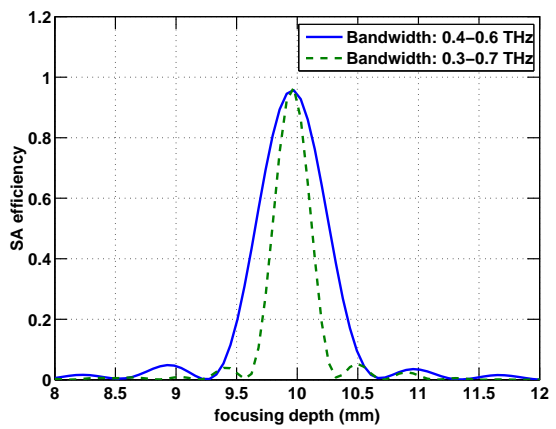

Figure 5.13: Depth of focus for two broadband averages. The blue curve corresponds to a broadband average of $0.4-0.6 \mathrm{THz}$, while the green curve corresponds to a broadband average from $0.3-0.7 \mathrm{THz}$. The $0.4 \mathrm{THz}$ broadband average improves depth resolution by $43 \%$.

used to approximate a point-scatterer. Two other ball bearing sizes (radii of 0.5 and $1.5 \mathrm{~mm}$ ) were also imaged with very similar results, therefore it is assumed that the depth resolution is the limiting factor and is much larger than the apparent size of the ball bearing.

\subsubsection{Broadband resolution improvement}

It will be shown here that broadband averaging significantly improves depth resolution. Depth isolation was previously demonstrated in the surface profile of the American dime in Section 5.3. Now, this improvement in depth resolution is quantified. The variable $C_{B B}$, as computed with (5.7), is shown in Figure 5.13 as a function of focusing depth (green). Such a curve is known as a depth profile.

The chosen band is $0.4-0.6 \mathrm{THz}$ for the solid blue curve. Also shown in Figure 5.13 is a depth profile (dashed green) with a broadband average of 0.3-0.7 
THz. One can readily see the improvement in depth resolution using broadband SA image processing. In addition to improved depth resolution, unwanted frequency dependent imaging artifacts are reduced. The FWHM for the $0.2 \mathrm{THz}$ bandwidth depth profile is $0.62 \mathrm{~mm}$ while the FWHM for the $0.4 \mathrm{THz}$ bandwidth depth profile is $0.35 \mathrm{~mm}$, an improvement in depth resolution by $43 \%$.

In addition to depth resolution improvement, there are multiple frequencydependent effects that can be mitigated. Both subjective speckle and side lobe artifacts are common in SAR, and vary across the band [86]. By using a broadband average, one considerably reduces both of these effects, which are not desired for most imaging applications.

\subsubsection{Depth localization}

An experiment was conducted to show that the SA system has proper relative depth localization. That is, objects focus to the correct depth relative to other targets. A steel ball bearing was lowered a distance $R_{z}$ of $9 \mathrm{~mm}, 10 \mathrm{~mm}$, and 11 mm below the virtual source. At each distance, the ball bearing was measured individually and then moved with a micrometer-driven z-stage. Although data sets at multiple depths are not recorded simultaneously, this experiment should provide a good indication that the SA focusing technique resolves to the correct depth.

Results for depth localization experiment are shown in Figure 5.14. The 


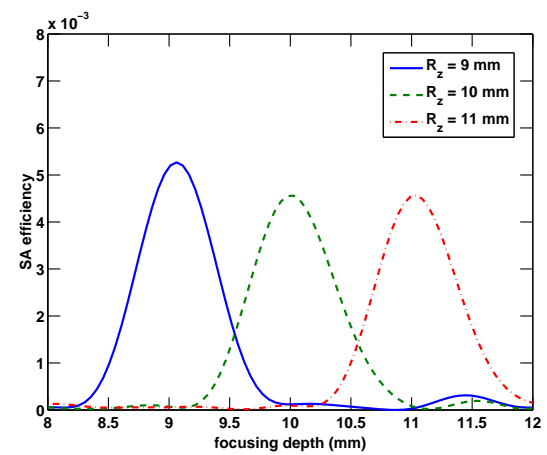

Figure 5.14: Depth of focus after broadband averaging from $0.3-0.5 \mathrm{THz}$ and intensity correction for single steel $1 \mathrm{~mm}$ ball bearing placed at three different depths: $9 \mathrm{~mm}$ (blue), $10 \mathrm{~mm}$ (green), and $11 \mathrm{~mm}$ (red).

three curves are depth profiles that correspond to the SA normalized intensity at the center of each ball bearing. The blue, green and red curves correspond to ball bearing positions of $9 \mathrm{~mm}, 10 \mathrm{~mm}$, and $11 \mathrm{~mm}$ below the virtual source, respectively. Each curve is generated by using a broadband average between 0.3 $\mathrm{THz}$ to $0.5 \mathrm{THz}$. One can clearly resolve between the multiple depths. The difference in location of peak SA focused amplitude between the $9 \mathrm{~mm}$ and 10 $\mathrm{mm}$ ball bearing is $0.962 \mathrm{~mm}$. The difference between the $10 \mathrm{~mm}$ and the $11 \mathrm{~mm}$ position is $1.068 \mathrm{~mm}$. Thus the depth localization is accurate to $40 \mu \mathrm{m}$, which is reasonable considering the broadband average of $0.3-0.5 \mathrm{THz}$ corresponds to 0.6-1.0 $\mathrm{mm}$ in wavelength. 


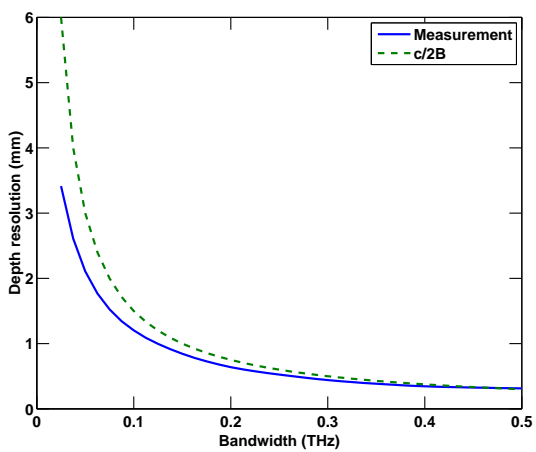

Figure 5.15: Depth resolution (in $\mathrm{mm}$ ) is plotted as a function of bandwidth included in the broadband summation from (5.7). The depth resolution is defined by the full-width at half max of the depth profile. Also plotted is the theoretical radar range resolution, given by the speed of light divided by twice the bandwidth.

\subsubsection{Bandwidth comparison}

As seen in Figure 5.13, a bandwidth increase of $0.2 \mathrm{THz}$ allows for a dramatically improved depth resolution. Clearly, the width of the broadband average plays a key role in determining the depth resolution in a 3-D SA image. Starting with a center frequency of $0.5 \mathrm{THz}$, the bandwidth was varied from 0 to $0.4 \mathrm{THz}$ in steps of $6.25 \mathrm{GHz}$. For example, a bandwidth of $0.2 \mathrm{THz}$ corresponds to a broadband average of $0.4-0.6 \mathrm{THz}$ in 32 steps. For each selected bandwidth, the broadband averaged depth profile was computed. To find the depth resolution, the FWHM was found from each depth profile.

Depth resolution is plotted as a function of bandwidth in Figure 5.15. At the far left end of the curve, the depth resolution is not impressive, only about $4 \mathrm{~mm}$. Looking at the far right end, a bandwidth of $0.5 \mathrm{THz}$ provides a depth resolution 
of just over $300 \mu \mathrm{m}$. However, a system with a bandwidth of $10 \mathrm{THz}$ should see depth resolution on the order of $15 \mu \mathrm{m}$, provided sufficient SNR and phase stability. Also plotted in Figure 5.15 is the theoretical RADAR range resolution,

$$
\Delta r=\frac{c}{2 B}
$$

The theoretical RADAR range resolution curve tracks well with the measured depth resolution. Notice the curve is inversely proportional to bandwidth, which is also consistent with optical coherence tomography and terahertz pulsed imaging. 


\section{Chapter 6}

\section{Synthetic-Aperture Based 3-D Spectral Imaging}

Having demonstrated 3-D broadband $\mathrm{THz}$ SA imaging capabilities, the focus shifts to chemical mapping and how it can be combined with 3-D imaging. Chapter 4 developed several advanced techniques for chemical detection, but didn't deal directly with the 3-D imaging problem. This chapter combines the two by presenting imaging formulations, followed by 3-D spectral surface profiling results using a two-sided pellet measured in the NEAR-Lab, with quantitative performance comparison between techniques.

\subsection{Theoretical formulations}

In this section, two different $3-\mathrm{D}$ spectral $\mathrm{THz}$ imaging techniques were developed, frequency differencing and correlation processing. Both provide concurrent 3-D spectral imaging suitable for a synthetic-aperture imaging configuration. Each was adapted from the original synthetic-aperture frequency-domain focusing, equations (5.4) and (5.7). 3-D spectral imaging techniques in this section are formed by the coherent addition of the electric field across frequency and sensor position. 


\subsubsection{Correlation processing}

Correlation processing can be used in conjunction with broadband syntheticaperture imaging, as both utilize the coherent summation of the electric field correlated with a exponential phase term [61]. The SA correlation processing output, $C P O_{S A}\left(\vec{r}_{h j l}\right)$, for each feature can be expressed as

$$
C P O_{S A}\left(\vec{r}_{h j l}\right)=\left|\frac{1}{Q} \sum_{q=1}^{Q} C_{N B}\left(\vec{r}_{h j l}, f_{q}\right) \times E_{C P}\left(f_{q}\right)\right|^{2}
$$

The correlation processing coherent average in (4.4) is now combined with the broadband synthetic-aperture imaging kernel used in (5.7). The bandwidth, $Q \Delta f$, is an important tuning parameter that influences detection performance metrics.

It was shown in the previous section that the phase-structured field in (6.1), $E_{C P}$, can be used to detect a resonance peak. However, adaptation to 3-D SA imaging is not trivial. Notice that the functional form of this term is the same as $e^{i 2 k d}$, a plane wave propagating some effective distance, $d$. Therefore, a correlation processed image is misfocused above the actual scene. However, CPO still minimizes returns corresponding to any spectrally flat material at it's true depth. To correct the misfocus that is incurred by (6.1), and provide concurrent 3-D imaging with good depth resolution, a broadband image from (5.7) was used to isolate the more significant facets from the scene. Doing this corrects 
for the misfocussing error, and mitigates unwanted artifacts incurred by the relatively narrow bandwidths needed in (6.1). In the case of the two-sided pellet, $C P O_{S A}\left(\vec{r}_{h j l}\right)$, is evaluated along the of the pellet surface; this is described further in Section 6.2.

\subsubsection{Frequency differencing}

Chapter 3 demonstrated how the negative derivative of the reflected amplitude can be used to identify materials in a difference image, as in Figure 4.5(b). This section outlines a simple method that can be used to adapt the difference image from using broadband SA processing. The derivative (or difference) operator cannot be used directly in conjunction with (5.7), as the electric field is complex. Taking the derivative of the electric field with respect to frequency would be applied to the phase in addition to the magnitude, which would corrupt imaging results.

However, features can be extracted by differencing two focused intensity images. Two broadband averages are taken using two center frequencies, $f_{c 1}$, and $f_{c 2}$, each with identical bandwidth, $\Delta f Q$. The SA frequency difference image, $C_{S A, d f}\left(\vec{r}_{h j l}\right)$, is given by

$$
C_{S A, d f}\left(\vec{r}_{h j l}\right)=\left.C_{B B}\left(\vec{r}_{h j l}\right)\right|_{f_{c 1}}-\left.C_{B B}\left(\vec{r}_{h j l},\right)\right|_{f_{c 2}},
$$




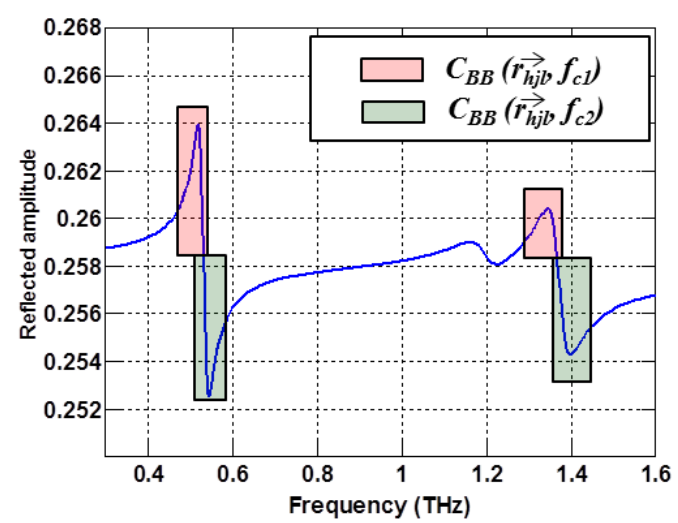

(a)

Figure 6.1: Reflected amplitude from lactose, with two highlighted frequency regions in red and green used to generate SA frequency difference images.

where $C_{B B}\left(\vec{r}_{h j l}, f_{c 1}\right)$ and $C_{B B}\left(\vec{r}_{h j l}, f_{c 2}\right)$ are the frequency averaged images centered at frequencies $f_{c 1}$ and $f_{c 2}$, as computed from (5.7). The variable, $Q$, is the total number of frequency bins involved in each average, and is an important tuning parameter that influences detection performance.

Frequency differencing is further explained using Figure 6.1, which shows the reflected amplitude for lactose. For the resonance peak at 0.53 , there are two frequency averages corresponding to center frequencies, $f_{c 1}$ and $f_{c 2}$, highlighted by red and green boxes, respectively. In this example, the frequency differencing technique accomplished by subtracting the focused image with $f_{c 2}=0.57 \mathrm{THz}$ from the focused image with $f_{c 1}=0.49 \mathrm{THz}$. There can be overlap between the two frequency averages if $Q \Delta f$ exceeds $f_{c 2}-f_{c 1}$. The choice of each of these parameters are dictated by the width of the resonance peaks. 


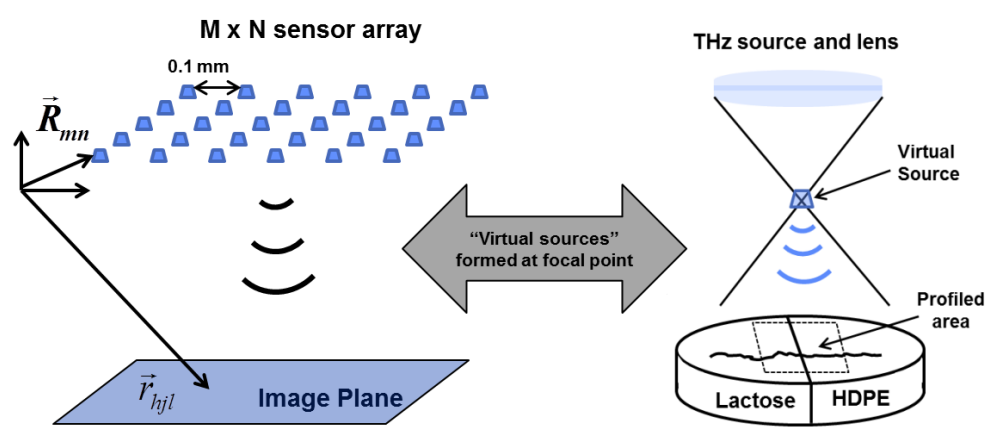

Figure 6.2: The two-sided pellet was lowered below the focal point (virtual source) of a one-inch lens and measured in a SA configuration. Only a square section in the center of the pellet was profiled.

\subsection{Spectral profiling results with two-sided pellet}

The two-sided pellet shown in Figure 6.3(b), made of lactose and HDPE, was imaged in a synthetic-aperture configuration. The top of the roughened pellet was lowered $1 \mathrm{~cm}$ below the focal point of a one-inch lens, as shown in Figure 6.2. By raster scanning the $\mathrm{THz}$ transceiver in both $x$ and $y$ directions, the complex scattered electric field was recorded for all sensor positions for a square patch of the pellet directly above the boundary between the two species. The square patch being profiled is outlined on the right side of Figure 6.2. First, a surface profile of the square section of pellet was created using a broadband 3-D image using the maximum value for $C_{B B}\left(\vec{r}_{h j l}\right)$ at all $x$ and $y$ locations, similarly to what was done in Section 5.3. Considering the rough surface of the pellet provides the largest refractive index mismatch, the largest value of $C_{B B}\left(\vec{r}_{h j l}\right)$ should occur at the surface. 


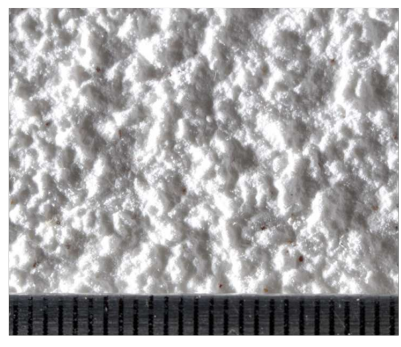

(a)

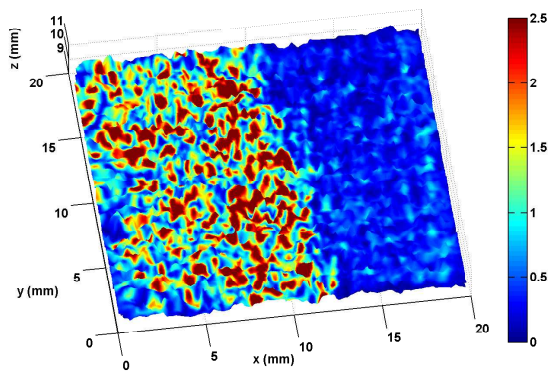

(c)

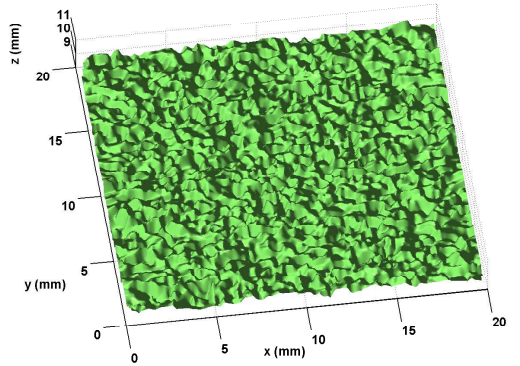

(b)

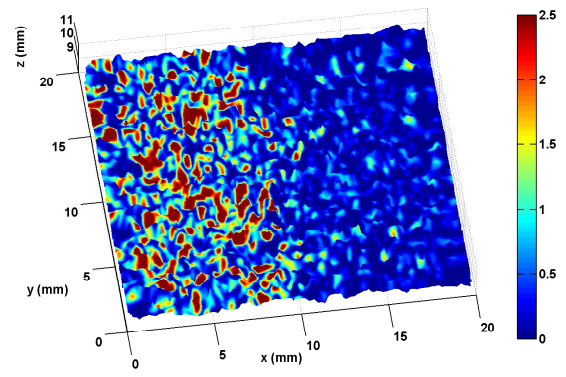

(d)

Figure 6.3: (a) Close in photo of a similar pellet constructed with lactose and high-density polyethylene. (b) Surface profile of the pellet obtained using (5.7). (c) Spectral surface profile of a square section of the two-sided pellet. The colorscale was created using correlation processing as computed from (6.1), normalized by its standard deviation. (c) Similar profile with colorscale created using the frequency differencing technique, as computed from (6.2), also normalized by its standard deviation.

The main goal for each image is to simultaneously display the scene and detect lactose using false color to indicate the species. Equations (6.1) and (6.2) were then evaluated along the surface, and used to determine the color for the 3-D THz spectral surface profiles. Figure 6.3(c) uses correlation processing as calculated from (6.1). Figure 6.3(d) shows a similar spectral profile using frequency differencing as calculated from (6.2). The red side of the colorscale on the right 
of each image denotes the 'brightest' values, most likely to be lactose. The color values are normalized to the standard deviation of each profile.

Figure 6.3(c) was computed using only the first lactose peak, two separate spectral regions $0.5-0.53 \mathrm{THz}$ and $0.53-0.56$, and summing contributions from each region incoherently. The broadband image used to generate the surface profile had a broadband average of $0.4-0.8 \mathrm{THz}$. The 3-D difference image from (6.2) does provide some depth isolation in addition to spectral imaging. However, for fair comparison, the same surface profile was used to create Figure 6.3(d). The difference profile was calculated using $f_{c 1}=0.52 \mathrm{THz}$, and $f_{c 2}=0.54$, with a bandwidth of $0.02 \mathrm{THz}$ for each image.

One can clearly distinguish between the two sides of the pellet in each figure. Clearly, $C P O_{S A}\left(\vec{r}_{h j l}\right)$ is able to visibly distinguish between the two species, lactose and HDPE. The frequency difference image, shown in Figure 6.3(d), also

reveals a difference between both sides. However, the difference profile is the weaker of the two in terms of detection performance, as will be shown quantitatively in the next section.

\subsubsection{Quantitative performance of spectral profiling}

Quantitative performance metrics were computed similarly to Figure 4.7(a), except each point on the surface profile was treated as a separate realization, instead of treating each 2-D pixel as a separate realization. An ROC curve is 


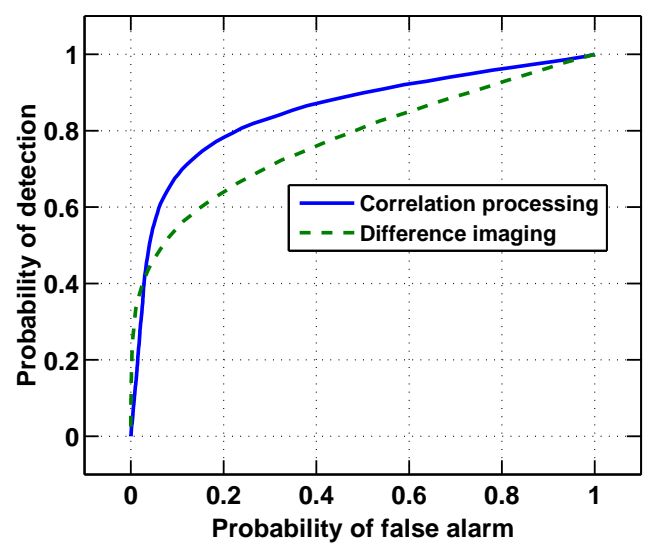

Figure 6.4: ROC curves comparing performance of THz SA spectral surface profiles using correlation processing from (6.1) and the frequency differencing technique from (6.2).

plotted in Figure 7.5, which compares the performance of both spectral profiling imaging techniques. The solid blue curve corresponds to correlation processing as computed from (6.1), and the dashed green curve corresponds to frequency differencing as computed from (6.2). Correlation processing performs the better of the two methods, with $P_{d}$ of 0.68 while maintaining a $P_{f a}$ of 0.1 . Frequency differencing produces a $P_{d}$ of 0.52 while constraining $P_{f a}$ to 0.1 .

After analyzing the spectral detection performance using a ROC curve, there is still one unanswered question pertaining to $\mathrm{THz}$ spectral surface profiling. That question is how accurate are the height profiles shown in Figures 6.3(d) and 6.3(c). Table 6.1 compares statistics for different profiling methods, all using Norton-brand 40 grit sandpaper.

The three independent groups/methods used to compute surface profiles were 
Table 6.1: Comparison of surface statistics for 40 grit sandpaper using various surface profiling techniques. The top two are considered more conventional, while the bottom two are new $\mathrm{THz}$ methods using two separate samples. * Average of three samples.

\begin{tabular}{|c|c|c|}
\hline & RMS height $(\mu \mathrm{m})$ & Correlation length $(\mu \mathrm{m})$ \\
\hline APL-UW $^{*}$ & $109 \mu \mathrm{m}$ & $441 \mu \mathrm{m}$ \\
\hline Nanovea $^{*}$ & $135 \mu \mathrm{m}$ & $420 \mu \mathrm{m}$ \\
\hline THz; rough pellet & $171 \mu \mathrm{m}$ & $385 \mu \mathrm{m}$ \\
\hline THz; gold-coated & $241 \mu \mathrm{m}$ & $363 \mu \mathrm{m}$ \\
\hline
\end{tabular}

UPL-UW, Nanovea, and the THz-based method presented in this dissertation. The top two sets of statistics correspond to UPL-UW and Nanovea. The bottom two are derived from a $\mathrm{THz}$ broadband SA 3-D image as computed from (5.7), corresponding to two samples, a rough pellet using 40 grit sandpaper to roughen the surface, and a gold-coated sandpaper sample. Each $\mathrm{THz}$ profile was created using a bandwidth of 0.4-0.8 THz.

The RMS heights for the $\mathrm{THz}$ methods were the highest of the four. The correlation lengths, however, are lower for the THz-measured samples compared to the APL-UW and Nanovea methods. It should be noted that the statistics generated by APL-UW and Nanovea were created using an average of the same three samples. The $\mathrm{THz}$ method used only one sample area for both the rough pellet and the gold-coated sandpaper sample with no average. While there is some variance from sample to sample, its possible $\mathrm{THz}$ radiation does not have the capability to accurately profile these type of surfaces. Considering that (5.12) 
provides a range resolution of $375 \mu \mathrm{m}$ for a bandwidth of $0.4 \mathrm{THz}$, profiling 40 grit sandpaper is pushing the limits of the system bandwidth. 


\section{Chapter 7}

\section{3-D THz Spectral Imaging of Dielectric Spheres}

Chapters 6 demonstrated THz SA spectral imaging capabilities in 3-D imaging configurations with pressed pellet measurements. In this chapter, simulations are used as an additional tool to investigate 3-D $\mathrm{THz}$ spectral imaging techniques. Two techniques are used to distinguish between two types of dielectric spheres, lactose, and HDPE. Those techniques are frequency differencing and matchedfield imaging. The theoretical formulation for the new technique, matched-field imaging, is first described. Then, simulation details and results are provided.

\subsection{Matched-field imaging}

A matched filter is a powerful technique in radar, communications, and infrared spectral imaging. With some slight modifications, it can be be applied to THz synthetic-aperture spectral imaging as well. A matched filter cross-correlates a measurement with conjugate model, summing over both frequency and sensor position. As mentioned earlier, a matched filter provides optimal SNR gain

in the case where the target signature is mixed with additive white Gaussian noise $[67,71]$. 
The matched filter expression from (4.3) will be now be combined with the SA imaging formulation from (5.7) to form the SA matched-field output, $C_{M F}\left(\vec{r}_{h j l}\right)$, which is given by

$$
C_{M F}\left(\vec{r}_{h j l}\right)=\frac{1}{Q} \sum_{q=1}^{Q} C_{N B}\left(\vec{r}_{h j l}, f_{q}\right) \times s_{0}^{*}\left(\left|R_{m n}-r_{h j l}\right|, f_{q}\right)
$$

where $\left|R_{m n}-r_{h j l}\right|$ is the Euclidian distance between the sensor located at $R_{m n}$, and the desired imaging pixel, $\vec{r}_{h j l}$. The notation, ${ }^{*}$, denotes the complex conjugate. The variable, $C_{N B}\left(\vec{r}_{h j l}, f_{q}\right)$, is the narrowband synthetic-aperture focused field from (5.4). The conjugate model, $s_{0}^{*}\left(R_{m n}-r_{h j l}, f_{q}\right)$, depends on this distance in addition to frequency, $f_{q}$, and incorporates both scattering information and $\mathrm{THz}$ spectral resonance features. Implied in (7.1) is an isotropic backscattering approximation, as $s_{0}^{*}\left(R_{m n}-r_{h j l}, f_{q}\right)$ does not depend on orientation between scatterer and sensor position. The variable, $Q$, is again the number of frequency bins included in the summation.

Typically, matched filters in spectral imaging require an amplitude spectrum be zero mean [72]. Therefore, prior to the evaluation of (7.1), the amplitude envelope is subtracted by a polynomial fit of the amplitude, and the phase is left intact. This serves both to ensure the amplitude signal is zero-mean, and reduce any global trend that might hinder detection performance. In the case of a single scattering dielectric sphere in the Raleigh regime, the backscattered electric field 
amplitude increases as a function of frequency.

While a wide bandwidth is required for good depth resolution, a relatively narrow bandwidth in (7.1) is desired for detection performance. To form a spectral 3-D image with good depth resolution, the SA matched-field output is multiplied by a weighting image, chosen to be a thresholded broadband SA image. That can be written as,

$$
\hat{C}_{M F}\left(\vec{r}_{h j l}\right)=C_{M F}\left(\vec{r}_{h j l}\right) \times\left[C_{B B}\left(\vec{r}_{h j l}\right)>\beta\right] .
$$

The value of $\left[C_{B B}\left(\vec{r}_{h j l}\right)>\beta\right]$ is zero unless $C_{B B}\left(\vec{r}_{h j l}\right)$ exceeds the threshold, $\beta$. This cleans up the final 3-D spectral image by eliminating sections of 3-D space that do not contain any objects or surfaces.

\subsection{Simulations details}

In this section, a Monte Carlo simulation is conducted in order to investigate the performance of 3-D broadband $\mathrm{THz}$ SA spectral imaging. The simulation consists of two parts, simulating the scattered (and received) electric field from two types of dielectric spheres, and then applying $\mathrm{THz}$ spectral detection algorithms to reconstruct the original scene while discriminating between different types of spheres. By creating a 3-D volume of spherical scatterers, insight can be gained on how various algorithms can effect depth resolution and performance 
metrics of the final 3-D image.

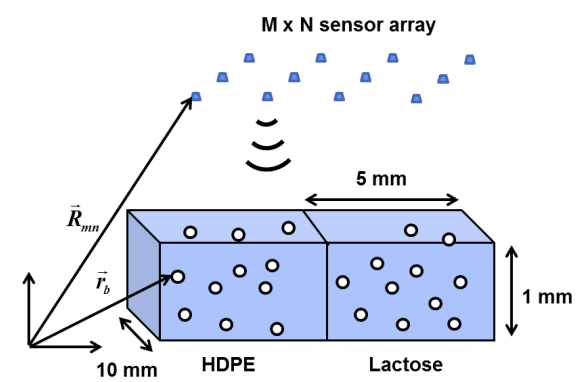

Figure 7.1: Monte Carlo configuration showing the locations of distributed dielectric spheres comprised of lactose or high-density polyethylene

Small dielectric spheres $(10 \mu \mathrm{m}$ in diameter $)$ were first randomly distributed in a volume with a narrow vertical extent. The background medium was assumed to be free space. The locations of all the scatterers have a Poisson distribution within a rectangular volume, $1 \mathrm{~mm}$ tall, as shown in Figure 7.1. Half the scatterers contained in the volume are comprised of HDPE, with the other half lactose. The index of refraction for HDPE is assumed to be 1.5, while the index of refraction for the lactose spheres was taken from a Lorentz oscillator model [12]. The simulated electric field was found by summing the complex field contributions from all individual scatterers, similar to [79]. Using a single scattering approximation, the comprehensive backscattered electric field received at each sensor, $E_{\text {sim }}\left(x_{n}, y_{m}, f_{q}\right)$, is given by

$$
E_{\text {sim }}\left(x_{n}, y_{m}, f_{q}\right)=\frac{1}{B} \sum_{b=1}^{B} e^{-i k\left|r_{b}-R_{m n}\right|} E_{\text {sphere }}\left(\left|r_{b}-R_{m n}\right|, f_{q}\right)
$$




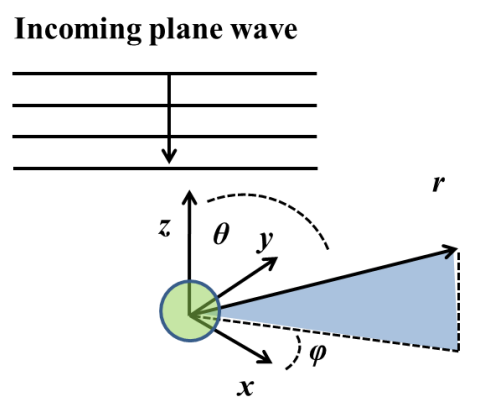

Figure 7.2: Geometry of a plane wave impinging on a sphere. The variable, $r$, describes vector between the center of the sphere and the observation point.

where $E_{\text {sphere }}\left(r, f_{q}\right)$ is the backscattered electric field from a dielectric sphere at a distance, $r$ and frequency $f_{q}$. The extra $e^{-i k r}$ term accounts for the wave propagating from the sensor position to the dielectric sphere. The backscattered electric field, $E_{\text {sphere }}\left(r, f_{q}\right)$, is computed as given in [87]. The geometry of the plane wave impinging on a dielectric sphere is shown in Figure. 7.2. The scattered electric field, $E_{\text {sphere }}(r)$, is given by

$$
E_{\text {sphere }}\left(r, f_{q}\right)=\left[\begin{array}{c}
E_{\theta} \\
E_{\rho}
\end{array}\right]=\frac{e^{-i k r}}{-i k r}\left[\begin{array}{cc}
S_{1} & 0 \\
0 & S_{2}
\end{array}\right] E_{i n},
$$

where the negative sign convention on the $e^{-i k r}$ term was chosen to match with pulsed $\mathrm{THz}$ measurements. The variable, $E_{\text {in }}$, is the incident scattered field (amplitude is set to unity). The complex scattering amplitudes, $S_{1}$ and $S_{2}$, are given as 


$$
S_{1}=\sum_{n=1}^{\infty} \operatorname{frac} 2 n+1 n(n+1)\left(a_{n} \pi_{n}+b_{n} \tau_{n}\right)
$$

and

$$
S_{2}=\sum_{n}^{\infty} \frac{2 n+1}{n(n+1)}\left(a_{n} \tau_{n}+b_{n} \pi_{n}\right) .
$$

The variables $\tau_{n}$ and $\pi_{n}$ are frequency and geometry dependent, and can be calculated recursively. The scattering coefficients, $a_{n}$ and $b_{n}$ are computed using Riccati-Bessel functions. A more detailed derivation is given here [87].

\subsection{3-D Spectral imaging results}

After generation of sphere locations using a random number generator in MATLAB, the comprehensive scattered electric field was evaluated according to (7.3). The locations of the dielectric spheres are shown for a single realization in Figure 7.3(a). The complex electric field data were applied to (5.7) to construct a $3-\mathrm{D} \mathrm{THz}$ broadband image, which is shown for the same realization in Figure 7.3(b).

Equations (7.2) and (6.2) were then used to produce a 3-D spectral image in each case. For fair comparison, a weighting image similar to the one used in (7.2) was applied to the frequency differencing technique. Similarly to 3-D spectral images in Chapter 6, each image was tuned to detect lactose, using false color. 


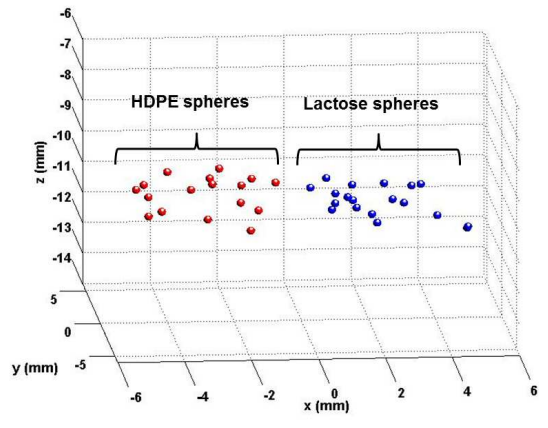

(a)

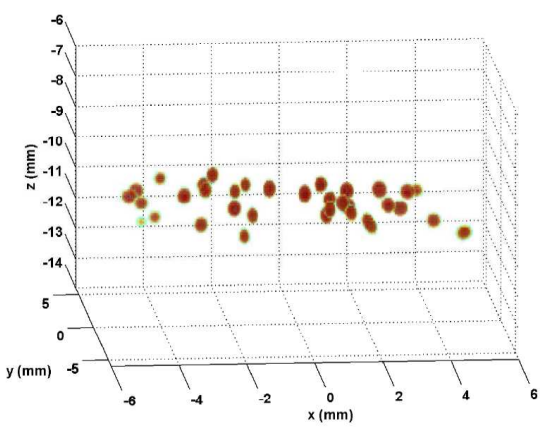

(b)

Figure 7.3: (a) Actual locations of dielectric spheres comprised of both highdensity polyethylene and lactose. (b) 3-D SA THz image of dielectric spheres using (5.7).

Figure 7.4 shows 3-D spectral imaging results, revealing both technique's ability to discern between types of spheres. Figure 7.4(a) shows a top-down view of a 3D spectral image using matched-field imaging, while Figure 7.4(b) uses frequency differencing. The red side of the colorscale on the right of each image denotes the 'brightest' voxel values, with a high likelihood of being lactose. The voxel values are normalized so that the maximum value in each image is unity.

The contrast between the two types of spheres is evident in each image, although not all images contain the same degree of contrast. The matched-field image produces the greatest distinction between the two species. The frequency difference image, shown in Figure 7.4(b), also reveals a difference between both sides. However, this image is the weaker of the two in terms of detection performance, as will be shown quantitatively in the next section. 


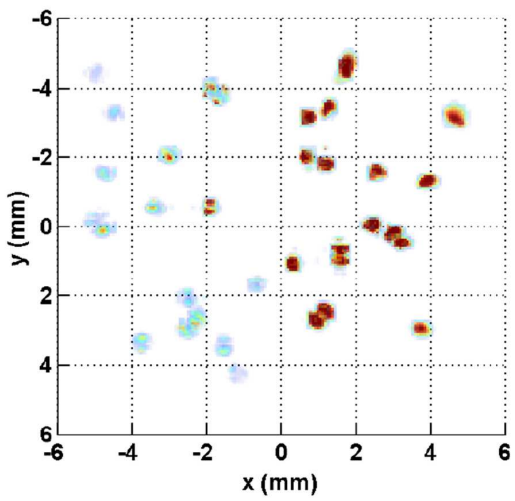

(a)

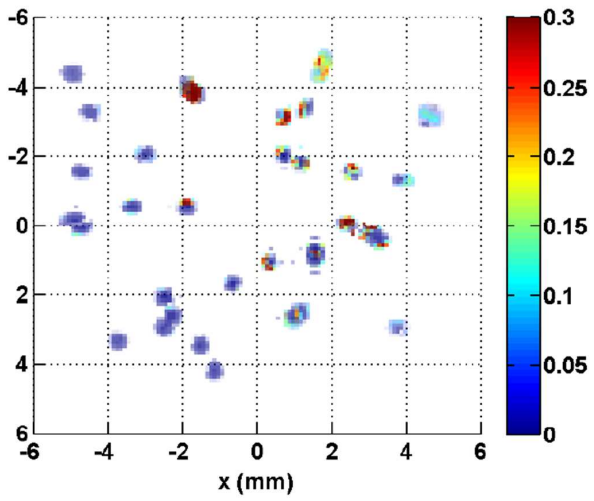

(b)

Figure 7.4: (a) Top-down $\mathrm{THz}$ spectral image of dielectric spheres comprised of high-density polyethylene and lactose using matched-field imaging from (7.2). (b) Spectral image using frequency differencing from (6.2).

\subsection{Quantitative performance comparison}

Quantitative performance metrics were computed using ROC analysis described in Chapter 4, except each 3-D voxel was treated as a separate realization, instead of treating each 2-D pixel as a separate realization. Recall that much of the 3-D spectral matched-field image has been forced to a zero value due to the weighting image applied in (7.2). To be counted as a detection or a false alarm, there must be a non-zero voxel value in $C_{B B}\left(\vec{r}_{h j l}\right)$.

Figure 7.5 shows a ROC curve that compares the performance of each imaging method quantitatively. 10 independent realizations were needed for the ROC curves to converge. Both matched-field imaging and frequency differencing perform well. The matched-field imaging technique performs better, especially when 


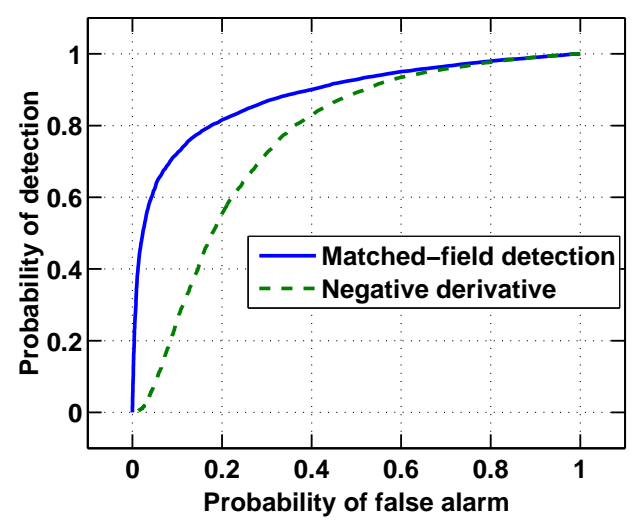

Figure 7.5: Receiver operating characteristic curves comparing performance of detection techniques corresponding to 3-D spectral images shown in Figure 7.4

constraining probability of false alarm to low values $\left(P_{f a}<0.2\right)$, which is realistic for many personnel screening applications. With $P_{f a}$ held constant at 0.1 , matched-field processing yields $P_{d}$ of 0.72 , while frequency differencing provides a $P_{d}$ of 0.27 . 


\section{Chapter 8}

\section{Conclusion and Future Work}

\subsection{Conclusion}

Throughout this dissertation, it has been demonstrated that $\mathrm{THz}$ imaging has considerable potential for 3-D chemical mapping. Every other portion of the spectrum has proved useful over time for a variety of applications from radar to biomedical optics and x-rays. There is no reason for the $\mathrm{THz}$ gap to be an exception to this. The advantages of $\mathrm{THz}$ radiation, often denoted as the 'last frontier' of the electromagnetic spectrum, are numerous:

1. THz frequencies can penetrate through a variety of packaging and clothing materials, while reflecting from more opaque materials such as human tissue.

2. $\mathrm{THz}$ wavelengths are small enough compared to radio wavelengths, so that $\mathrm{THz}$ systems are capable of producing higher resolution images than mircowaves or millimeter waves.

3. Many chemical materials, such as drugs and explosives, contain unique spectral signatures observable in both transmission and reflection mode. 


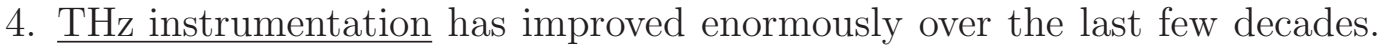
Source power and receiver sensitivity have seen large increases, while the manufacturing size and cost of each source and receiver continues to fall over time.

For these reasons, $\mathrm{THz} 3-\mathrm{D}$ spectral imaging of explosive and other chemicals is both realizable and powerful. In particular, reflection 3-D spectral imaging could be useful for personnel and mail screening, helping to secure military checkpoints and airports around the world. The pharmaceutical industry also has shown interest in this type of technology for use as a quality screening tool in manufacturing processes.

This dissertation has explored multiple 3-D THz imaging methods, emphasizing synthetic-aperture (SA) imaging. Synthetic-aperture imaging has the potential to isolate depth in a 3-D image without sacrificing frequency resolution necessary to detect drugs or explosives. Additionally, improvement in depth resolution is achievable using broadband $\mathrm{THz}$ SA image processing. Broadband averaging was shown to dramatically increase depth resolution of the 3-D images demonstrated in simulation and with images of real-world objects.

One large component of this work was to develop and test SA imaging techniques. This was done using various objects, such as an American dime. However, 
the ultimate goal was to test algorithms on materials with interesting spectral features. This involved constructing 3-D samples containing the explosive simulant, lactose, and applying the spectral imaging techniques outlined in this proposal to provide chemical mapping. In addition, multiple types of simulations were used for development of spectral imaging algorithms, and for gaining intuition on how they operate.

Specifically, the following items were completed as a part of this dissertation.

1. Studied rough surface effects on of the scattered signature of lactose observed from a multiposition sensor array. This study, which served as the first of it's kind, illustrated the challenges of diffuse $\mathrm{THz}$ scattering spectroscopy, and how to overcome them. It became clear in this study that a multiposition array was the best way to view a scattering signature, and has become further utilized in a SA imaging configuration.

2. Implemented a broadband 3-D THz SA imaging algorithm. This implementation was the first of its kind, utilizing a 2-D array and to create high resolution 3 - D THz images. Using a broadband $\mathrm{THz}$ system will allow 3-D imaging with significant depth and lateral resolution. This algorithm was based on a modified version of conventional synthetic-aperture radar techniques.

3. Applied advanced image processing algorithms to measured and simulated 
scattered electric field data from pressed pellets and numerically generated dielectric spheres and rough surfaces.

4. Designed and validated a hollow dielectric tube waveguide suitable for broadband single-mode propagation at THz frequencies. This waveguide design utilizes the $H E M_{11}$ mode, which supports single-mode propagation for subwavelength fibers for several octaves. As part of this work, analytic expressions were developed, and compared results with numerical simulation and measurement.

Through each chapter of this dissertation, each of these algorithms was motivated, demonstrated, and the results were discussed. Each of the techniques have been proven to be useful, although they are not without disadvantage.

\subsection{Future work}

The following areas can be considered for further research related to work on THz 3-D SA imaging:

1. Measure scattered response from other chemicals, such as C-4, that contain multiple resonance features in the $\mathrm{THz}$ range, and apply detection techniques, such as correlation processing, on scattered data from such chemicals. 
2. Model scattered response from rough surface using more complex numerical analysis tools. One particularly powerful tool for $\mathrm{THz}$ pulses is finitedifference-time-domain software, such as MEEP. This model can be incorporated in the matched-field imaging formulation from Chapter 7.

3. Develop 3-D THz SA imaging for use with other more complex environments, such as layered structures or curved surfaces .

\subsection{Contributions to the $\mathrm{THz}$ community}

Many of the items covered in this work have formed an important contribution to the $\mathrm{THz}$ community, this research involved in this dissertation has produced nine conference and journal publications. The full list of these publications is shown below.

1. S.C. Henry, L.M. Zurk, S. Schecklman, THz Imaging and Spectral Profiling Using Correlation Processing, submitted to IEEE Transactions on Terahertz Science \& Technology.

2. S.C. Henry, G. Kniffin, L.M. Zurk, 3-D Terahertz Spectral Imaging of Dielectric Spheres, in preparation to be submitted to Optics Letters.

3. S.C. Henry, G. Kniffin, L.M. Zurk, S. Schecklman, 3-D Broadband Terahertz 
Synthetic Aperture Imaging, $37^{\text {th }}$ International Conference on Infrared, Terahertz and Millimeter Waves, September, 2012.

4. S.C. Henry, L.M. Zurk, S. Schecklman Terahertz Spectral Imaging Using Correlation Processing, $37^{\text {th }}$ International Conference on Infrared, Terahertz and Millimeter Waves, September, 2012.

5. S.C. Henry, L.M. Zurk, D.D. Duncan, S. Schecklman, Three-dimensional Broadband Terahertz Synthetic Aperture Imaging, Optical Engineering, vol. 51, no. 9, 2012.

6. S. Schecklman, L.M. Zurk, S.C. Henry, G. P. Kniffin, Terahertz Material Detection From Diffuse Surface Scattering, Journal of Applied Physics, vol. 109, no. 9, 2011.

7. L. M. Zurk, S.C. Henry, D. Duncan, and S. Schecklman, Physics-based processing for terahertz reflection spectroscopy and imaging, Proceedings of SPIE - The International Society for Optical Engineering, vol. 7854, 2010.

8. G. Sundberg, L.M. Zurk, S. Schecklman, S. C. Henry,Modeling RoughSurface and Granular Scattering at Terahertz Frequencies Using the FiniteDifference Time-Domain Method, IEEE Geoscience, Oct. 2010.

9. S.C. Henry, G. Kniffin, S. Schecklman, and L. M. Zurk, Measurement and Modeling of Rough Surface Effects on Terahertz Spectroscopy, Proceedings 
of SPIE - The International Society for Optical Engineering, vol. 7601, 2010.

10. S.C. Henry, L.M. Zurk, R.L. Campbell, P. Hanaway, Measurement and modeling of dielectric tube waveguides for terahertz pulses, Proceedings of SPIE - The International Society for Optical Engineering, vol. 7215, 2009.

Although the measured spectral imaging results shown throughout this dissertation only uses on a single lactose feature at $0.53 \mathrm{THz}$, these same techniques could be used on several resonances throughout the $\mathrm{THz}$ range to detect a materials of interest substance such as RDX and HMX explosive compounds, which contains numerous features between 0.5 and $3 \mathrm{THz}$. At the current pace, future THz imaging systems will have sufficient SNR and bandwidths to utilize features higher in the $\mathrm{THz}$ range. While $\mathrm{THz} 3-\mathrm{D}$ spectral imaging has enormous potential in security applications, techniques like those presented in this dissertation are needed to capture the full potential of dynamic $\mathrm{THz}$ time-domain systems. 


\section{References}

[1] Y.-C. Shen, P. F. Taday, D. A. Newnham, M. C. Kemp, and M. Pepper, "3d chemical mapping using terahertz pulsed imaging," vol. 5727, San Jose, CA, United states, 2005, pp. 24 - 31. [Online]. Available: http://dx.doi.org/10.1117/12.591472

[2] S. Mickan and X.-C. Zhang, "T-ray sensing and imaging," International Journal of High Speed Electronics and Systems, vol. 13, no. 2, pp. 601 - 676, 2003. [Online]. Available: http://dx.doi.org/10.1142/S0129156403001843

[3] J. Hasch, E. Topak, R. Schnabel, T. Zwick, R. Weigel, and C. Waldschmidt, "Millimeter-wave technology for automotive radar sensors in the 77 ghz frequency band," IEEE Transactions on Microwave Theory and Techniques, vol. 60, no. 3, pp. 845 - 860, 2012. [Online]. Available: http://dx.doi.org/10.1109/TMTT.2011.2178427

[4] L. Rakotondrainibe, Y. Kokar, G. Zaharia, and G. El Zein, "Millimeter-wave system for high data rate indoor communications," Piscataway, NJ, USA, 2009. [Online]. Available: http://dx.doi.org/10.1109/ISSCS.2009.5206120

[5] R. Wilson, "Millimeter and sub-millimeter wave technology for astronomy: From early bell labs work to alma," Piscataway, NJ, USA, 2011. [Online]. Available: http://dx.doi.org/10.1109/irmmw-THz.2011.6104781

[6] V. A. Trofimov and N. V. Peskov, "About possibility of eliminating the influence of water vapour on the detection and identification of substance at using the thz laser pulse," vol. 8119, San Diego, CA, United states, 2011. [Online]. Available: http://dx.doi.org/10.1117/12.893084

[7] X. Lu, B. Clough, I.-C. Ho, J. Liu, J. Dai, and X.-C. Zhang, "Recent progress of thz generation and detection in ambient air or gases," vol. 7917, San Francisco, CA, United states, 2011. [Online]. Available: http://dx.doi.org/10.1117/12.876848

[8] L. Zurk, G. Sundberg, S. Schecklman, Z. Zhou, A. Chen, and E. Thorsos, "Scattering effects in terahertz reflection spectroscopy," 
vol. 6949, Orlando, FL, United states, 2008. [Online]. Available: http://dx.doi.org/10.1117/12.784222

[9] S. Schecklman, L. M. Zurk, S. Henry, and G. P. Kniffin, "Terahertz material detection from diffuse surface scattering," Journal of Applied Physics, vol. 109, no. 9, p. 094902, 2011. [Online]. Available: http://link.aip.org/link/?JAP/109/094902/1

[10] S. Henry, S. Schecklman, G. Kniffin, L. Zurk, and A. Chen, "Measurement and modeling of rough surface effects on terahertz spectroscopy," vol. 7601, San Francisco, CA, United states, 2010, pp. The Society of Photo-Optical Instrumentation Engineers (SPIE) -. [Online]. Available: http://dx.doi.org/10.1117/12.841054

[11] L. Zurk, S. Henry, S. Schecklman, and D. Duncan, "Physics-based processing for terahertz reflection spectroscopy and imaging," vol. 7854, Beijing, China, 2010, pp. The Society of Photo-Optical Instrumentation Engineers (SPIE); Chinese Optical Society (COS). [Online]. Available: http://dx.doi.org/10.1117/12.870664

[12] G. Kniffin and L. Zurk, "Model-based material parameter estimation for terahertz reflection spectroscopy," IEEE Transactions on THz, 2012.

[13] R. K. A. T. Ulaby, Fawwaz T.;Moore, Microwave Remote Sensing. Artech House, Inc., 1986.

[14] W. Ibrahim and H. M. Algabroun, "The family tree of breast microwave imaging techniques," vol. 21 IFMBE, no. 1, Kuala Lumpur, Malaysia, 2008, pp. 258 - 261. [Online]. Available: http://dx.doi.org/10.1007/978-3-54069139-6-67

[15] S. M. Salvador, E. C. Fear, M. Okoniewski, and J. R. Matyas, "Microwave imaging of the knee: On sensitivity, resolution and multiple tears detection," Banff, AB, Canada, 2009. [Online]. Available: http://dx.doi.org/10.1109/ANTEMURSI.2009.4805109

[16] M. Kemp, P. Taday, B. Cole, J. Cluff, A. Fitzgerald, and W. Tribe, "Security applications of terahertz technology," vol. 5070, Orlando, FL, United states, 2003, pp. 44 - 52. [Online]. Available: http://dx.doi.org/10.1117/12.500491

[17] J. Hildenbrand, J. Herbst, J. Wollenstein, and A. Lambrecht, "Explosive detection using infrared laser spectroscopy," vol. 7222, San Jose, CA, United states, 2009. [Online]. Available: http://dx.doi.org/10.1117/12.808976 
[18] M. Nordberg, M. Akeson, H. Ostmark, and T. E. Carlsson, "Standoff detection of explosive particles by imaging raman spectroscopy," vol. 8017, Orlando, FL, United states, 2011, pp. The Society of Photo-Optical Instrumentation Engineers (SPIE). [Online]. Available: http://dx.doi.org/10.1117/12.883179

[19] J. K. Olsen, A. Greve, N. Privorotskaya, L. Senesac, T. Thundat, W. King, and A. Boisen, "Micro-calorimetric sensor for trace explosive particle detection," vol. 7679, Orlando, FL, United states, 2010, pp. The Society of Photo-Optical Instrumentation Engineers (SPIE). [Online]. Available: http://dx.doi.org/10.1117/12.850492

[20] W. R. Tribe, D. A. Newnham, P. F. Taday, and M. C. Kemp, "Hidden object detection: Security applications of terahertz technology," vol. 5354, San Jose, CA, United states, 2004, pp. 168 - 176. [Online]. Available: http://dx.doi.org/10.1117/12.543049

[21] H. Zhong, C. Zhang, L. Zhang, Y. Zhao, and X.-C. Zhang, "A phase feature extraction technique for terahertz reflection spectroscopy," Applied Physics Letters, vol. 92, no. 22, 2008.

[22] M. Yamashita, T. Kiwa, M. Tonouchi, and K. Kawase, "Evaluation of spatial resolution in laser-terahertz emission microscope for inspecting electric faults in integrated circuits," vol. 5354, San Jose, CA, United states, 2004, pp. 104 - 111. [Online]. Available: http://dx.doi.org/10.1117/12.528833

[23] S. Wietzke, C. Jansen, C. Jordens, N. Krumbholz, N. Vieweg, M. Scheller, M. Shakfa, D. Romeike, T. Hochrein, M. Mikulics, and M. Koch, "Industrial applications of thz systems," in Proc. SPIE, vol. 7385, Beijing, China, 2009, p. 738506. [Online]. Available: http://dx.doi.org/10.1117/12.840991

[24] K. Fukunaga, Y. Ogawa, S. Hayashi, and I. Hosako, "Application of terahertz spectroscopy for character recognition in a medieval manuscript," IEICE Electronics Express, vol. 5, no. 7, pp. 223 - 228, 2008. [Online]. Available: http://dx.doi.org/10.1587/elex.5.223

[25] K. Fukunaga, I. Hosako, M. Picollo, and Y. Kohdzuma, "Application of thz sensing to analysis of works of art for conservation," Montreal, QC, Canada, 2010, pp. 147 - 150. [Online]. Available: http://dx.doi.org/10.1109/MWP.2010.5664137

[26] J. Jackson, J. Labaune, G. Mourou, L. D'Alessandro, A. Whyte, and M. Menu, "Pulsed terahertz investigation of corroded and mineralized 
copper alloy historical artifacts," Houston, TX, United states, 2011. [Online]. Available: http://dx.doi.org/10.1109/irmmw-THz.2011.6104844

[27] J. Labaune, J. Jackson, S. Pages-Camagna, M. Menu, and G. Mourou, "Terahertz investigation of egyptian artifacts," Rome, Italy, 2010. [Online]. Available: http://dx.doi.org/10.1109/ICIMW.2010.5612354

[28] D. Etayo, J. Iriarte, I. Palacios, J. Teniente, I. Ederra, and R. Gonzalo, "Active thz inspection of water content in plants," vol. 7670, Orlando, FL, United states, 2010, pp. The Society of Photo-Optical Instrumentation Engineers (SPIE) -. [Online]. Available: http://dx.doi.org/10.1117/12.849990

[29] R. S. Singh, Z. D. Taylor, P. Tewari, D. Bennett, M. O. Culjat, H. Lee, E. R. Brown, and W. S. Grundfest, "Thz imaging of skin hydration: Motivation for the frequency band," vol. 7555, San Francisco, CA, United states, 2010, pp. The Society of Photo-Optical Instrumentation Engineers (SPIE). [Online]. Available: http://dx.doi.org/10.1117/12.845820

[30] T. Yasui, T. Yasuda, K.-I. Sawanaka, and T. Araki, "Terahertz paintmeter for noncontact monitoring of thickness and drying progress in paint film," Applied Optics, vol. 44, no. 32, pp. 6849 - 6856, 2005. [Online]. Available: http://dx.doi.org/10.1364/AO.44.006849

[31] P. Ashworth, E. Pickwell-MacPherson, E. Provenzano, S. Pinder, A. Purushotham, M. Pepper, and V. Wallace, "Terahertz pulsed spectroscopy of freshly excised human breast cancer," Optics Express, vol. 17, no. 15, pp. 12444 - 54, 2009. [Online]. Available: http://dx.doi.org/10.1364/OE.17.012444

[32] A. Markelz, "Terahertz dielectric sensitivity to biomolecular structure and function," IEEE Journal on Selected Topics in Quantum Electronics, vol. 14, no. 1, pp. 180 - 90, 2008. [Online]. Available: http://dx.doi.org/10.1109/JSTQE.2007.913424

[33] T. W. Crowe, W. L. Bishop, D. W. Porterfield, J. L. Hesler, and R. M. Weikle II, "Opening the terahertz window with integrated diode circuits," vol. 40, no. 10, 2005, pp. 2104 - 2109. [Online]. Available: http://dx.doi.org/10.1109/JSSC.2005.854599

[34] R. Czarny, M. Alouini, C. Larat, S. Dhillon, M. Krakowsky, S. Bansropun, V. Ortiz, C. Sirtori, B. Gerard, and D. Dolfi, "Continuous wave thz generation through photomixing using a dual-frequency yb3+:kgd(wo4)2 
laser," vol. 5619, London, United kingdom, 2004, pp. 198 - 207. [Online]. Available: http://dx.doi.org/10.1117/12.581285

[35] S. Matsuura, M. Tani, H. Abe, K. Sakai, H. Ozeki, and S. Saito, "High-resolution terahertz spectroscopy by a compact radiation source based on photomixing with diode lasers in a photoconductive antenna," Journal of molecular spectroscopy, vol. 187, no. 1, pp. 97 - 101, 1998. [Online]. Available: http://dx.doi.org/10.1006/jmsp.1997.7486

[36] S. Preu, F. Renner, M. Hanson, T. Wilkinson, S. Malzer, A. Gossard, E. Brown, G. Dohler, and L. Wang, "Efficient thz source using gaas and ingaas nipnip photomixers," Munich, Germany, 2007. [Online]. Available: http://dx.doi.org/10.1109/CLEOE-IQEC.2007.4385998

[37] D. Saeedkia, A. Majedi, S. Safavi-Naeini, and R. Mansour, "A cw photoconductive integrated photomixer/antenna thz source," vol. 5354, San Jose, CA, United states, 2004, pp. 94 - 103. [Online]. Available: http://dx.doi.org/10.1117/12.528258

[38] K. S. E. M. Grischkowsky, D. and C. Fattinger, "Far-infrared time-domain spectroscopy with thz beams of dielectrics and semiconductors," JOSA-B, vol. 7, pp. 2006-2015, 1990.

[39] Z. Piao, M. Tani, and K. Sakai, "Carrier dynamics and terahertz radiation in photoconductive antennas," Japanese Journal of Applied Physics, Part 1: Regular Papers and Short Notes and Review Papers, vol. 39, no. 1, pp. 96 100, 2000.

[40] A. Hulsmann, A. Liebelt, A. Tessmann, A. Leuther, M. Schlechtweg, and O. Ambacher, "Active millimeter-wave imaging using raster scanner," vol. 7308, Orlando, FL, United states, 2009, p. The International Society for Optical Engineering (SPIE). [Online]. Available: http://dx.doi.org/10.1117/12.813388

[41] A. Lettington, N. Alexander, and D. Dunn, "A new opto-mechanical scanner for millimeter and sub-millimeter wave imaging," vol. 5789, Orlando, FL, United states, 2005, pp. 16 - 23. [Online]. Available: http://dx.doi.org/10.1117/12.597745

[42] K. L. Nguyen, M. L. Johns, L. F. Gladden, C. H. Worrall, P. Alexander, S. Barbieri, H. E. Beere, D. A. Ritchie, and E. H. Linfield, "Threedimensional imaging with a terahertz quantum cascade laser," in Proc. High Frequency Postgraduate Student Colloquium, 2005, pp. 101-104. 
[43] K. B. Cooper, R. J. Dengler, N. Llombart, T. Bryllert, G. Chattopadhyay, E. Schlecht, J. Gill, C. Lee, A. Skalare, I. Mehdi, and P. H. Siegel, "Penetrating 3-d imaging at 4- and 25-m range using a submillimeter-wave radar," vol. 56, no. 12, 2008, pp. 2771 - 2778. [Online]. Available: http://dx.doi.org/10.1109/TMTT.2008.2007081

[44] C. Zhang, K. Mu, X. Jiang, Y. Jiao, L. Zhang, Q. Zhou, Y. Zhang, J. Shen, G. Zhao, and X.-C. Zhang, "Identification of explosives and drugs inspection of material defects with thz radiation," vol. 6840, Beijing, China, 2008, p. The International Society for Optical Engineering (SPIE); Chinese Optical Society (COS). [Online]. Available: http://dx.doi.org/10.1117/12.760133

[45] J. A. Zeitler and L. F. Gladden, "In-vitro tomography and non-destructive imaging at depth of pharmaceutical solid dosage forms," European Journal of Pharmaceutics and Biopharmaceutics, vol. 71, no. 1, pp. 2 - 22, 2009. [Online]. Available: http://www.sciencedirect.com/science/article/pii/S0939641108003287

[46] R. M. Woodward, "Terahertz technology in biological and chemical sensing for defence," vol. 5617, London, United kingdom, 2004, pp. 341 - 352. [Online]. Available: http://dx.doi.org/10.1117/12.569427

[47] D. Arnone, "Terahertz pulsed imaging and spectroscopy for chemical detection and security," vol. 1, Williamsburg, VA, United states, 2005, pp. 198-205.

[48] F. Shen and Y.-B. Ying, "Applications of terahertz spectroscopy and imaging techniques in food safety inspection," Guang Pu Xue Yu Guang Pu Fen Xi/Spectroscopy and Spectral Analysis, vol. 29, no. 6, pp. 1445 - 1449, 2009. [Online]. Available: http://dx.doi.org/10.3964/j.issn.10000593(2009)06-1445-05

[49] F. M. Henderson and Z.-G. Xia, "SAR applications in human settlement detection, population estimation and urban land use pattern analysis: a status report," IEEE Transactions on Geoscience and Remote Sensing, vol. 35, no. 1, pp. 79-85, 1997.

[50] S. Cloude and E. Pottier, "An entropy based classification scheme for land applications of polarimetric SAR," Geoscience and Remote Sensing, IEEE Transactions on, vol. 35, no. 1, pp. 68-78, 1997.

[51] B. K. Mohan, B. B. Madhavan, and U. M. D. Gupta, "Integration of IRS-1A L2 data by fuzzy logic approaches for landuse classification." J1 - International Journal of Remote Sensing, vol. 21, no. 8, pp. 1709-1723, 2000. 
[52] Y.-C. Lin and K. Sarabandi, "Retrieval of forest parameters using a fractalbased coherent scattering model and a genetic algorithm," Geoscience and Remote Sensing, IEEE Transactions on, vol. 37, no. 3, pp. 1415-1424, 1999.

[53] M. Sato and T. Koike, "Classification of tree types by polarimetric pi-SAR," in 2003 IEEE IGARSS: Learning From Earth's Shapes and Colours, Jul 2125 2003, vol. 1, Toulouse, France, 2003, pp. 431-433.

[54] R. Magagi, M. Bernier, and C.-H. Ung, "Quantitative analysis of RADARSAT SAR data over a sparse forest canopy," Geoscience and Remote Sensing, IEEE Transactions on, vol. 40, no. 6, pp. 1301-1313, 2002.

[55] M. Dawson, A. Fung, and M. Manry, "A robust statistical-based estimator for soil moisture retrieval from radar measurements," Geoscience and Remote Sensing, IEEE Transactions on, vol. 35, no. 1, pp. 57-67, 1997.

[56] P. Dubois, J. van Zyl, and T. Engman, "Measuring soil moisture with imaging radars," Geoscience and Remote Sensing, IEEE Transactions on, vol. 33, no. 4, pp. 915-926, 1995.

[57] G. Satalino, F. Mattia, M. Davidson, T. L. Toan, G. Pasquariello, and M. Borgeaud, "On current limits of soil moisture retrieval from ERS-SAR data," Geoscience and Remote Sensing, IEEE Transactions on, vol. 40, no. 11, pp. 2438-2447, 2002.

[58] J. O'Hara and D. Grischkowsky, "Quasi-optic synthetic phased-array terahertz imaging," Journal of the Optical Society of America B: Optical Physics, vol. 21, no. 6, pp. 1178 - 1191, 2004. [Online]. Available: http://dx.doi.org/10.1364/JOSAB.21.001178

[59] A. Goltsman and A. I. Zaghloul, "Three-dimensional interferometric imaging at terahertz frequencies using three-dimensional spiral array," in Proc. 33rd Int. Conf. Infrared, Millimeter and Terahertz Waves IRMMW-THz 2008, 2008, pp. 1-2.

[60] V. Krozer, T. Loffler, J. Dall, A. Kusk, F. Eichhorn, R. K. Olsson, J. D. Buron, P. U. Jepsen, V. Zhurbenko, and T. Jensen, "Terahertz imaging systems with aperture synthesis techniques," IEEE Transactions on Microwave Theory and Techniques, vol. 58, no. 7 PART 2, pp. 2027 - 2039, 2010. [Online]. Available: http://dx.doi.org/10.1109/TMTT.2010.2050246

[61] S. Henry, "3-d broadband thz synthetic aperture imaging," SPIE Optical Engineering, vol. 51, no. 9, pp. 091603-1, 2012. 
[62] B. Ferguson, S. Wang, D. Gray, D. Abbot, and X.-C. Zhang, "T-ray computed tomography," Opt. Lett., vol. 27, no. 15, pp. 1312-1314, Aug 2002. [Online]. Available: http://ol.osa.org/abstract.cfm?URI=ol-27-15-1312

[63] X. Yin, B. W.-H. Ng, J. A. Zeitler, K. L. Nguyen, L. F. Gladden, and D. Abbott, "Local computed tomography using a thz quantum cascade laser," IEEE Sensors Journal, vol. 10, pp. 1718 - 1731, 2010. [Online]. Available: http://dx.doi.org/10.1109/JSEN.2010.2045754

[64] M. Imamura, S. Nishina, A. Irisawa, T. Yamashita, and E. Kato, "3d imaging and analysis system using terahertz waves," Rome, Italy, 2010. [Online]. Available: http://dx.doi.org/10.1109/ICIMW.2010.5612459

[65] N. Sunaguchi, Y. Sasaki, N. Maikusa, M. Kawai, T. Yuasa, and C. Otani, "Depth-resolving thz imaging with tomosynthesis," Optics Express, vol. 17, no. 12, pp. 9558 - 9570, 2009. [Online]. Available: http://dx.doi.org/10.1364/OE.17.009558

[66] M. I. Skolnik, Radar Handbook. McGraw-Hill Professional, 1990.

[67] D. Manolakis and G. Shaw, "Detection algorithms for hyperspectral imaging applications," IEEE Signal Processing Magazine, vol. 19, no. 1, pp. 29 - 43, 2002. [Online]. Available: http://dx.doi.org/10.1109/79.974724

[68] T. Lillesand and R. Kiefer, Remote Sensing and Image Interpretation, 2nd, Ed. Wiley, 1987.

[69] K. Kawase, Y. Ogawa, Y. Watanabe, and H. Inoue, "Non-destructive terahertz imaging of illicit drugs using spectral fingerprints," Optics Express, vol. 11, no. 20, pp. 2549 - 2554, 2003.

[70] A. D. Stocker and A. P. Schaum, "Application of stochastic mixing models to hyperspectral detection problems," vol. 3071, Orlando, FL, USA, 1997, pp. $47-60$.

[71] S. Kay, Fundamentals of Statistical Signal Processing. Englewood Cliffs,: NJ: Prentice Hall, 1998.

[72] D. Manolakis, R. Lockwood, T. Cooley, and J. Jacobson, "Robust matched filters for hyperspectral target detection," Denver, CO, United states, 2006, pp. 368 - 370. [Online]. Available: http://dx.doi.org/10.1109/IGARSS.2006.99 
[73] J. E. Reaugh, "Grain-scale dynamics in explosives," University of California, Lawrence Livermore National Laboratory, Technical Information Department, Livermore, CA 94551, 2002.

[74] G. Sundberg, L. M. Zurk, S. Schecklman, and S. Henry, "Modeling rough-surface and granular scattering at terahertz frequencies using the finite-difference time-domain method," IEEE Transactions on Geoscience and Remote Sensing, vol. 48, no. 10, pp. 3709 - 3719, 2010. [Online]. Available: http://dx.doi.org/10.1109/TGRS.2010.2048717

[75] H. Liu, Y. Chen, G. J. Bastiaans, and X.-C. Zhang, "Diffuse fresnel reflection spectroscopy of explosive rdx studied by thz time-domain spectroscopy," in Proc. Joint 30th Int. Conf. Infrared and Millimeter Waves and 13th Int. Conf. Terahertz Electronics IRMMW-THz 2005, vol. 2, 2005, pp. 588-589.

[76] C. Konek, J. Wilkinson, O. Esenturk, E. Heilweil, and M. Kemp, "Terahertz spectroscopy of explosives and simulants - rdx, petn, sugar and l-tartaric acid," vol. 7311, 2009. [Online]. Available: http://dx.doi.org/10.1117/12.817913

[77] Nanovea, 6 Morgan, Suite 156, Irvine, CA 92618, Tech. Rep., www.nanovea.com.

[78] P. Pedersen and A. Grebe, "Measurement of rough surface scattering using time delay spectrometry," Proceedings of the IEEE Ultrasonics Symposium, vol. 1, pp. $827-831,1997$.

[79] G. Zhang and L. Tsang, "Angular correlation function and scattering coefficient of electromagnetic waves scattered by a buried object under a twodimensional rough surface," vol. 3, Seattle, WA, USA, 1998, pp. 1466 - 1468.

[80] P. Beckmann and A. Spizzichino, The Scattering of Electromagnetic Waves from Rough Surfaces. Boston: Artech House, 1987.

[81] K. Yamamoto, M. Yamaguchi, F. Miyamaru, M. Tani, M. Hangyo, T. Ikeda, A. Matsushita, K. Koide, M. Tatsuno, and Y. Minami, "Noninvasive inspection of c-4 explosive in mails by terahertz timedomain spectroscopy," Japanese Journal of Applied Physics, Part 2 (Letters), vol. 43, no. 3B, pp. 414 - 17, 2004/03/15. [Online]. Available: http://dx.doi.org/10.1143/JJAP.43.L414

[82] D. E. Wahl, Spotlight-Mode Synthetic Aperture Radar: A Signal Processing Approach. Springer, 1996. 
[83] Z. Zhang and T. Buma, "Adaptive terahertz imaging using a virtual transceiver and coherence weighting," Optics Express, vol. 17, no. 20, pp. 17812 - 17817, 2009. [Online]. Available: http://dx.doi.org/10.1364/OE.17.017812

[84] Y. Zhang and W. H. Abdulla, "A comparative study of time-delay estimation techniques using microphone arrays," Department of Electrical and Computer Engineering, The University of Auckland, Tech. Rep., 2005.

[85] D. Mittleman, S. Hunsche, L. Boivin, and M. Nuss, "T-ray tomography," Optics Letters, vol. 22, no. 12, pp. 904 - 6, 1997/06/15.

[86] C. Lopez-Martinez and X. Fabregas, "Multidimensional speckle noise reduction in synthetic aperture radar images," Barcelona, Spain, 2008, pp. 176 179. [Online]. Available: http://dx.doi.org/10.1109/IGARSS.2007.4422758

[87] C. F. Bohren and D. Huffman, Absorption and scattering of light by small particles. Wiley, 1983.

[88] A. Fenn, D. Temme, W. Delaney, and W. Courtney, "The development of phased-array radar technology," Lincoln Laboratory Journal, vol. 12, no. 2, pp. $321-40,2000 / /$.

[89] K. Wang and D. M. Mittleman, "Metal wires for terahertz wave guiding," Nature, vol. 432, no. 7015, pp. 376 - 379, 2004. [Online]. Available: http://dx.doi.org/10.1038/nature03040

[90] J. Dai, J. Zhang, W. Zhang, and D. Grischkowsky, "Terahertz time-domain spectroscopy characterization of the far-infrared absorption and index of refraction of high-resistivity, float-zone silicon," Journal of the Optical Society of America B: Optical Physics, vol. 21, no. 7, pp. 1379 - 1386, 2004. [Online]. Available: http://dx.doi.org/10.1364/JOSAB.21.001379

[91] G. L. Yip and T. Auyeung, "Lauching efficiency of the he//1//1 surface-wave mode on a dielectric tube." IEEE Transactions on Microwave Theory and Techniques, vol. MTT-22, no. 1, pp. 6 - 14, 1974. [Online]. Available: http://dx.doi.org/10.1109/TMTT.1974.1128154

[92] C. Yeh and G. Lindgren, "Computing the propagation characteristics of radially stratified fibers: An efficient method." Applied Optics, vol. 16, no. 2, pp. $483-493,1977$.

[93] C. A. Balanis, Advanced Engineering Electromagnetics. John Wiley and Sons, 1989. 
[94] H. Green, "The radiation pattern of a conical horn," Journal of Electromagnetic Waves and Applications, vol. 20, no. 9, pp. 1149 - 1160, 2006. [Online]. Available: http://dx.doi.org/10.1163/156939306777442999

[95] J. Birch, "Far infrared optical constants of polyethylene," Infrared Physics, vol. 30, no. 2, pp. 195 -, 1990. [Online]. Available: http://dx.doi.org/10.1016/0020-0891(90)90031-P

[96] C. A. Balanis, "Advanced engineering electromagnetics," AEU, Archiv fuer Elektronik und Uebertragungstechnik: International Journal of Electronics and Communications, vol. 49, no. 4, pp. 236 -, 1995.

[97] M. Nagel, A. Marchewka, and H. Kurz, "Low-index discontinuity thz waveguides," Piscataway, NJ 08855-1331, United States, 2006, p. 569. [Online]. Available: http://dx.doi.org/10.1109/ICIMW.2006.368777

[98] V. R. Almeida, Q. Xu, C. A. Barrios, and M. Lipson, "Guiding and confining light in void nanostructure," Optics Letters, vol. 29, no. 11, pp. 1209 1211, 2004. [Online]. Available: http://dx.doi.org/10.1364/OL.29.001209 


\section{Appendix A}

\section{Study on Dielectric Tube Waveguides}

The focus of the dissertation has been on utilizing broadband $\mathrm{THz}$ pulses for 3-D imaging and spectroscopy. Not much was discussed on the generation and confinement of a broadband $\mathrm{THz}$ pulse without distortion. Moreover, physical phased arrays, which operate similarly to synthetic arrays described in Chapter 5, require a controllable phase shift at every element. This can be achieved by a network of switchable waveguides [88]. This section summarizes a study that was conducted on the feasibility of hollow dielectric tube waveguides that will permit the undistorted and low-loss transmission of broadband $\mathrm{THz}$ pulses for use in a variety of applications.

While $\mathrm{THz}$ imaging has great promise, most $\mathrm{THz}$ systems have no guiding mechanism and therefore suffer spherical spreading loss with current free space techniques. A waveguide would eliminate spreading losses, have low attenuation and dispersion (same phase delay across frequency), and high field confinement. Effective terahertz spectroscopy also requires especially large bandwidths to identify spectral signatures; therefore single-mode propagation is necessary to transmit broadband pulses. Single-mode waveguides can be achieved by utilizing the fundamental $H E M_{11}$ mode, which has no cutoff frequency, or by suppressing higher order modes.

Several waveguides have been proposed for $\mathrm{THz}$ frequencies. Scaled metal waveguides used for millimeter-wave frequencies (less than $300 \mathrm{GHz}$ ) can be 
used, but single mode propagation is not possible beyond one octave. In some applications, spectra of interest span several octaves or even decades, resulting in intermodal interference. Although, some attempts have been made to strip higher order modes using directional couplers and surface roughness. If such higher order modes could be minimized, metallic waveguides could be utilized for spectroscopy. More recently, the Sommerfeld wire has received considerable interest. The Sommerfeld wire consists of a single metal wire that carries electromagnetic energy on its surface due to the high index discontinuity. Though it is very non-dispersive; the electric field distribution extends quite far in the radial direction. [89] A summary of potential terahertz waveguides is included in Table A.1.

Simple dielectric fibers can also be used to transport $\mathrm{THz}$ energy, though they can exhibit high attenuation due to the heating loss associated with dielectric material. Many dielectric materials that are commonly used in optical waveguides become quite lossy in the $\mathrm{THz}$ regime $(0.3-3.0 \mathrm{THz})$, and exploration of low loss materials is an area of active research. [90]

Table A.1: List of possible THz structures

\begin{tabular}{|c|c|c|}
\hline Type of Waveguide & Frequency Range & THz scaling difficulty \\
\hline Optical Fiber & Optics & Lossy \\
\hline Metal Waveguide & Microwaves & Band-Limited \\
\hline Microstrip & Microwaves & Loosely Bound \\
\hline Sommerfeld Wire & Terahertz & Radiation \\
\hline
\end{tabular}

In this section, cylindrical, hollow-core, dielectric waveguides are presented for the G-band (140-220 GHz). Propagation characteristics were obtained by theoretical analysis, computer simulation, and experiments conducted using a vector 


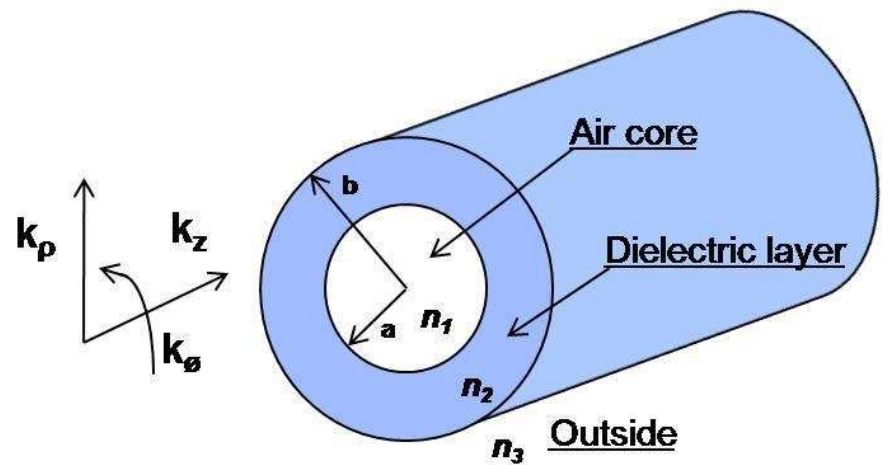

Figure A.1: Hollow dielectric waveguide showing all three regions. The axial wave vector, $k_{z}$, depends on $a, b, n_{2}$, and frequency. Cylindrical coordinate system is used here due to the symmetries involved in matching boundary conditions.

network analyzer (VNA) with a cylindrical horn attachment. The hollow dielectric waveguide consists of a cylindrical dielectric rod with a hollow air core. It has been shown that the air core lowers attenuation and dispersion when compared to a dielectric rod. [91] Also, by decreasing the size of the air core, high field confinement can be achieved. Field confinement is in an important parameter in the areas such as signal integrity where fields that extend far beyond the surface of the waveguide can interfere with the surrounding environment. This appendix first presents background propagation physics and compares theoretical predictions with transmission measurements taken from 140-220 GHz. Concluding the appendix, an improved waveguide more suitable for higher $\mathrm{THz}$ frequencies is considered.

\section{A.1 Propagation Physics}

The main goal of this section is to be able to quickly predict the performance of the waveguide for given physical parameters. These parameters are the inner 
radius, $a$, the outer radius, $b$, and $n_{2}$, the index of refraction of the dielectric material in layer 2. The basic diagram of a hollow, dielectric waveguide is shown in Figure A.1, where the center region is air, that is, $n_{1}=n_{3}=1$ and $\mu=\mu_{0}$ for all layers. Finding the performance of the waveguide requires computing modal solutions, each with a unique complex propagation constant, $k_{z}=\beta_{z}-j \alpha_{z}$, where $\beta_{z}$ is the axial wave number in units of radians $/ \mathrm{m}$, and $\alpha_{z}$ is the attenuation rate in units of $m^{-1}$. Finding suitable values of $k_{z}$ is commonly referred to as an eigenvalue solution to the waveguide problem.

Once $k_{z}$ is found, then all fields can be solved for any hybrid mode, $H E_{n m}$ or $E H_{n m}$, where $n$ is the order of the all Bessel functions, and $k_{z}$ is the $m^{\text {th }}$ solution to the eigenvalue problem, which will be obtained by matching boundary conditions at both surfaces. First, we must express the fields in cylindrical coordinates in terms of physical parameters and arbitrary coefficients. [92] However, using the basis representation found in [93], the tangential fields can be matched at all boundaries, namely $E_{\phi}(\rho), E_{z}(\rho), H_{\phi}(\rho), H_{z}(\rho)$.

The tangential field expressions in the air core are

$$
\left[\begin{array}{c}
\bar{E}_{z}^{1}(\rho) \\
\bar{H}_{z}^{1}(\rho) \\
\bar{E}_{\phi}^{1}(\rho) \\
\bar{H}_{\phi}^{1}(\rho)
\end{array}\right]=\left[\begin{array}{cccc}
J_{n}\left(k_{\rho}^{1} \rho\right) & 0 & 0 & 0 \\
0 & J_{n}\left(k_{\rho}^{1} \rho\right) & 0 & 0 \\
\frac{j n k_{z}}{\rho k_{\rho}^{12}} J_{n}\left(k_{\rho}^{1} \rho\right) & \frac{j \omega \mu_{0}}{k_{\rho}^{1}} J_{n}^{\prime}\left(k_{\rho}^{1} \rho\right) & 0 & 0 \\
-\frac{j \omega \epsilon_{1}}{k_{\rho}^{1}} J_{n}^{\prime}\left(k_{\rho}^{1} \rho\right) & -\frac{j n k_{z}}{\rho k_{\rho}^{12}} J_{n}\left(k_{\rho}^{1} \rho\right) & 0 & 0
\end{array}\right]\left[\begin{array}{c}
A_{1} \\
B_{1} \\
0 \\
0
\end{array}\right]=M_{A}(\rho)\left[\begin{array}{c}
A_{1} \\
B_{1} \\
0 \\
0
\end{array}\right]
$$

where $A_{i}, B_{i}$, etc. are coefficients for the $i^{\text {th }}$ layer, and $J_{n}$ is a Bessel function of order n. The notation for the field quantities $(E, H)$ is that the superscript 
denotes the region (i.e., $E^{1}$ is the electric field in the air core, etc.). The permittivities's for each layer are denoted as $\epsilon_{i}$, and are equal to the square of the index of refraction (i.e., $\epsilon_{1}=n_{1}^{2}$, etc.). There is an additional $\phi$ dependence term, either $\sin (n \phi)$ or $\cos (n \phi)$ for each field component. These terms are omitted here because they are identical for each layer and will cancel when matching boundary conditions. In the dielectric layer, the tangential field expressions are

$$
\begin{gathered}
{\left[\begin{array}{c}
\bar{E}_{z}^{2}(\rho) \\
\bar{H}_{z}^{2}(\rho) \\
\bar{E}_{\phi}^{2}(\rho) \\
\bar{H}_{\phi}^{2}(\rho)
\end{array}\right]=\left[\begin{array}{cccc}
J_{n}\left(k_{\rho}^{2} \rho\right) & Y_{n}\left(k_{\rho}^{2} \rho\right) & 0 & 0 \\
0 & 0 & J_{n}\left(k_{\rho}^{2} \rho\right) & Y_{n}\left(k_{\rho}^{2} \rho\right) \\
\frac{j n k_{z}}{\rho k_{\rho}^{2}} J_{n}\left(k_{\rho}^{2} \rho\right) & \frac{j n k_{z}}{\rho k_{\rho}^{2}} Y_{n}\left(k_{\rho}^{2} \rho\right) & \frac{j \omega \mu_{0}}{k_{\rho}^{2}} J_{n}^{\prime}\left(k_{\rho}^{2} \rho\right) & \frac{j \omega \mu_{0}}{k_{\rho}^{2}} Y_{n}^{\prime}\left(k_{\rho}^{2} \rho\right) \\
-\frac{j \omega \epsilon_{2}}{k_{\rho}^{2}} J_{n}^{\prime}\left(k_{\rho}^{2} \rho\right) & -\frac{j \omega \epsilon_{2}}{k_{\rho}^{2}} Y_{n}^{\prime}\left(k_{\rho}^{2} \rho\right) & -\frac{j n k_{z}}{\rho k_{\rho}^{2}} J_{n}\left(k_{\rho}^{2} \rho\right) & -\frac{j n k_{z}}{\rho k_{\rho}^{2}} Y_{n}\left(k_{\rho}^{2} \rho\right)
\end{array}\right]\left[\begin{array}{c}
A_{2} \\
B_{2} \\
C_{2} \\
D_{2}
\end{array}\right]} \\
=M_{B}(\rho)\left[\begin{array}{c}
A_{2} \\
B_{2} \\
C_{2} \\
D_{2}
\end{array}\right],
\end{gathered}
$$

where $Y_{n}$ is the Bessel function of the second kind of order $n$. Prime denotes the derivative operator with respect to the argument of the Bessel function. In the outside region, the tangential field expressions are

$$
\left[\begin{array}{c}
\bar{E}_{z}^{3}(\rho) \\
\bar{H}_{z}^{3}(\rho) \\
\bar{E}_{\phi}^{3}(\rho) \\
\bar{H}_{\phi}^{3}(\rho)
\end{array}\right]=\left[\begin{array}{cccc}
K_{n}\left(\alpha_{\rho} \rho\right) & 0 & 0 & 0 \\
0 & K_{n}\left(\alpha_{\rho} \rho\right) & 0 & 0 \\
\frac{j n k_{z}}{\rho \alpha_{\rho}{ }^{2}} K_{n}\left(\alpha_{\rho} \rho\right) & \frac{j \omega \mu_{0}}{\alpha_{\rho}} K_{n}^{\prime}\left(\alpha_{\rho} \rho\right) & 0 & 0 \\
-\frac{j \omega \epsilon_{3}}{\alpha_{\rho}} K_{n}^{\prime}\left(\alpha_{\rho} \rho\right) & -\frac{j n k_{z}}{\rho \alpha_{\rho}{ }^{2}} K_{n}\left(\alpha_{\rho} \rho\right) & 0 & 0
\end{array}\right]\left[\begin{array}{c}
A_{3} \\
B_{3} \\
0 \\
0
\end{array}\right]=M_{C}(\rho)\left[\begin{array}{c}
A_{3} \\
B_{3} \\
0 \\
0
\end{array}\right]
$$

and the variables $M_{A}, M_{B}, M_{C}$ are are shorthand notation denoting the $4 \times 4$ matrices containing all of the Bessel functions. $K_{n}$ denotes the modified Bessel 
function of the second kind of order $n$. However, these equations alone are not enough to solve the eigenvalue problem. The radial wave numbers $, k_{\rho}^{1}, k_{\rho}^{2}, \alpha_{\rho}$, can be related to $k_{z}$ using

$$
\begin{gathered}
k_{\rho}^{1}=\sqrt{\left(n_{0} k_{0}\right)^{2}-\left(k_{z}\right)^{2}}, \\
k_{\rho}^{2}=\sqrt{\left(n_{1} k_{0}\right)^{2}-\left(k_{z}\right)^{2}}, \\
\alpha_{\rho}=\sqrt{\left(k_{z}\right)^{2}-\left(n_{0} k_{0}\right)^{2}}, \\
k_{0}=\frac{2 \pi f}{c},
\end{gathered}
$$

and

$$
n_{0} k_{0}<k_{z}<n_{1} k_{0}
$$

Note that $\alpha_{\rho}$ corresponds to radial field attenuation (not to be confused with $\alpha_{z}$, the axial attenuation rate). When $\alpha_{\rho}$ is real, then $K_{n}\left(\alpha_{\rho} \rho\right)$ represents the evanescently decaying fields outside the outer boundary. When $\alpha_{\rho}$ is imaginary, then $K_{n}\left(\alpha_{\rho} \rho\right)$ represents cylindrically spreading waves. The surface between the air core (region 1) and dielectric layer (region 2) results in the following boundary conditions,

$$
\left[\begin{array}{c}
\bar{E}_{z}^{1}(\rho=a) \\
\bar{H}_{z}^{1}(\rho=a) \\
\bar{E}_{\phi}^{1}(\rho=a) \\
\bar{H}_{\phi}^{1}(\rho=a)
\end{array}\right]=\left[\begin{array}{c}
\bar{E}_{z}^{2}(\rho=a) \\
\bar{H}_{z}^{2}(\rho=a) \\
\bar{E}_{\phi}^{2}(\rho=a) \\
\bar{H}_{\phi}^{2}(\rho=a)
\end{array}\right]
$$

and

and 


$$
M_{A}(\rho=a)\left[\begin{array}{c}
A_{1} \\
B_{1} \\
0 \\
0
\end{array}\right]=M_{B}(\rho=a)\left[\begin{array}{c}
A_{2} \\
B_{2} \\
C_{2} \\
D_{2}
\end{array}\right] .
$$

The surface between the dielectric layer (region 2) and the outside (region 3) results in the following boundary condition:

$$
\left[\begin{array}{c}
\bar{E}_{z}^{2}(\rho=b) \\
\bar{H}_{z}^{2}(\rho=b) \\
\bar{E}_{\phi}^{2}(\rho=b) \\
\bar{H}_{\phi}^{2}(\rho=b)
\end{array}\right]=\left[\begin{array}{c}
\bar{E}_{z}^{3}(\rho=b) \\
\bar{H}_{z}^{3}(\rho=b) \\
\bar{E}_{\phi}^{3}(\rho=b) \\
\bar{H}_{\phi}^{3}(\rho=b)
\end{array}\right],
$$

and

$$
M_{B}(\rho=b)\left[\begin{array}{c}
A_{2} \\
B_{2} \\
C 2 \\
D 2
\end{array}\right]=M_{C}(\rho=b)\left[\begin{array}{c}
A_{3} \\
B_{3} \\
0 \\
0
\end{array}\right] .
$$

By combining $M_{A}, M_{B}, M_{C}$, we can avoid solving simultaneously for coefficients, and solve for $k_{z}$ directly. Rewriting (A.12), we have

$$
\left[\begin{array}{c}
A_{2} \\
B_{2} \\
C_{2} \\
D_{2}
\end{array}\right]=M_{B}^{-1}(\rho=b) M_{C}(\rho=b)\left[\begin{array}{c}
A_{3} \\
B_{3} \\
0 \\
0
\end{array}\right],
$$

and by inserting (A.10), we can write 


$$
M_{A}(\rho=a)\left[\begin{array}{c}
A_{1} \\
B_{1} \\
0 \\
0
\end{array}\right]=M_{B}(\rho=a) M_{B}^{-1}(\rho=b) M_{C}(\rho=b)\left[\begin{array}{c}
A_{3} \\
B_{3} \\
0 \\
0
\end{array}\right],
$$

and

$$
\left[\begin{array}{cccc}
M_{A 11} & M_{A 12} & 0 & 0 \\
M_{A 21} & M_{A 22} & 0 & 0 \\
M_{A 31} & M_{A 32} & 0 & 0 \\
M_{A 41} & M_{A 42} & 0 & 0
\end{array}\right]\left[\begin{array}{c}
A_{1} \\
B_{1} \\
0 \\
0
\end{array}\right]=\left[\begin{array}{llll}
m_{11} & m_{12} & 0 & 0 \\
m_{21} & m_{22} & 0 & 0 \\
m_{31} & m_{32} & 0 & 0 \\
m_{41} & m_{42} & 0 & 0
\end{array}\right]\left[\begin{array}{c}
A_{3} \\
B_{3} \\
0 \\
0
\end{array}\right] .
$$

By subtracting all elements on the right, we get

$$
\left[\begin{array}{cccc}
M_{A 11} & M_{A 12} & -m_{11} & -m_{12} \\
M_{A 21} & M_{A 22} & -m_{21} & -m_{22} \\
M_{A 31} & M_{A 32} & -m_{31} & -m_{32} \\
M_{A 41} & M_{A 42} & -m_{41} & -m_{42}
\end{array}\right]\left[\begin{array}{c}
A_{1} \\
B_{1} \\
A_{3} \\
B_{3}
\end{array}\right]=0
$$

We can now call this new 4x4 matrix $M_{D}\left(k_{z}\right)$. Physical parameters $a, b, n_{2}$, and $f$ all influence $M_{D}\left(k_{z}\right)$, though $k_{z}$ is the undetermined variable. If $M_{D}$ is an invertible $4 \times 4$ matrix, then we have no problem solving the characteristic equation numerically. That is, we set the determinant of $M_{D}\left(k_{z}\right)$ to zero,

$$
\operatorname{det}\left(M_{D}\left(k_{z}\right)\right)=0 .
$$

\section{A.1.1 Horn Excitation}

The measurements presented in the following section were conducted using conical horn extensions. Thus, in this section we account for the non-uniform 


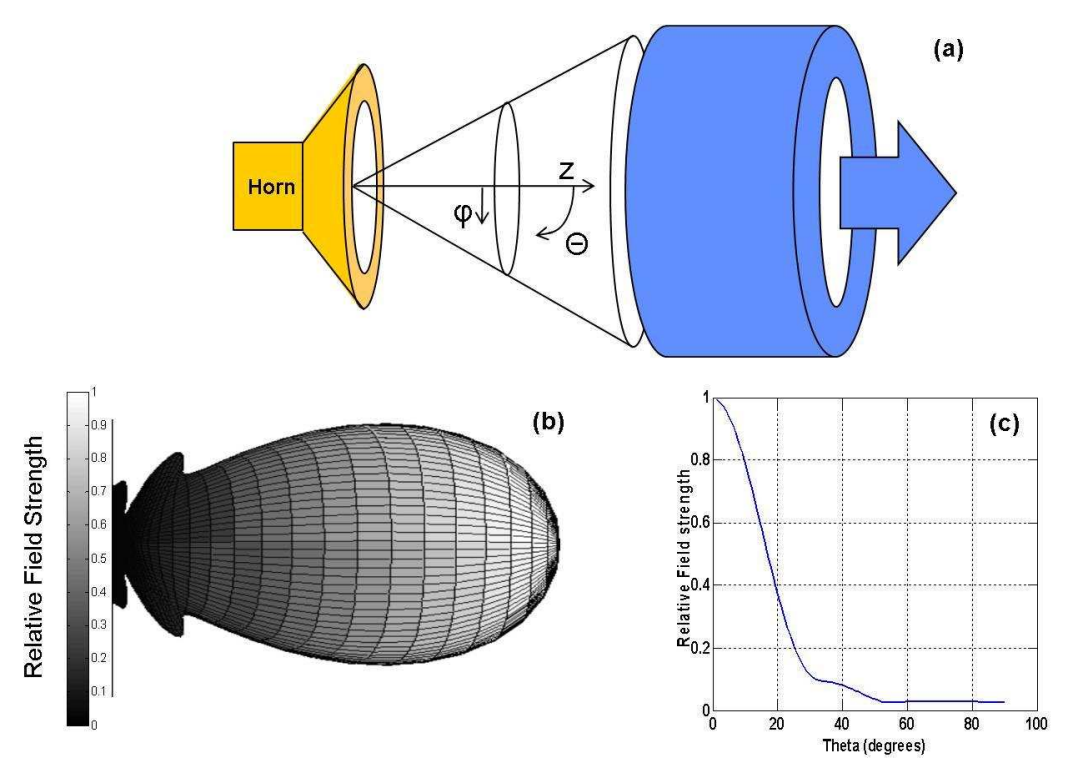

Figure A.2: The horn attachment will filter higher order modes. (a) The diagram of the horn aperture with some launch angle, $\theta_{l}$, exciting the waveguide. (a) Antenna beam pattern for the conical horn attachment. (c) Mode weighting dependence on $\theta_{l}$.

mode excitation introduced by the horn. Before we compute an expected response for the transmission coefficient, $S_{21}$, we must first properly weight each mode. The approximation maps antenna beam pattern for the horn antenna onto the aperture of the waveguide, as shown in Figure A.2. First, analytical expressions for the conical horn antenna must be applied. [94] Our horn has dimensions 0.75 $\mathrm{mm}$ (inside radius) $\times 2.37 \mathrm{~mm}$ (outside radius) $\times 6.11 \mathrm{~mm}$ (length) The electric field beam pattern will be denoted as $F_{E}(\phi, \theta)$. The conical horn has fairly good axial symmetry, but it is not perfect due to the vertical polarization of the $T E_{10}$ mode launched by the horn. Therefore, we must integrate over all $\phi$ in order to determine the average mode weighting function, $\mathrm{c}(\theta)$, which is shown in the plot in Figure A.2 (c). We can write each coupling coefficient, $c_{l}\left(\theta_{l}\right)$, as 

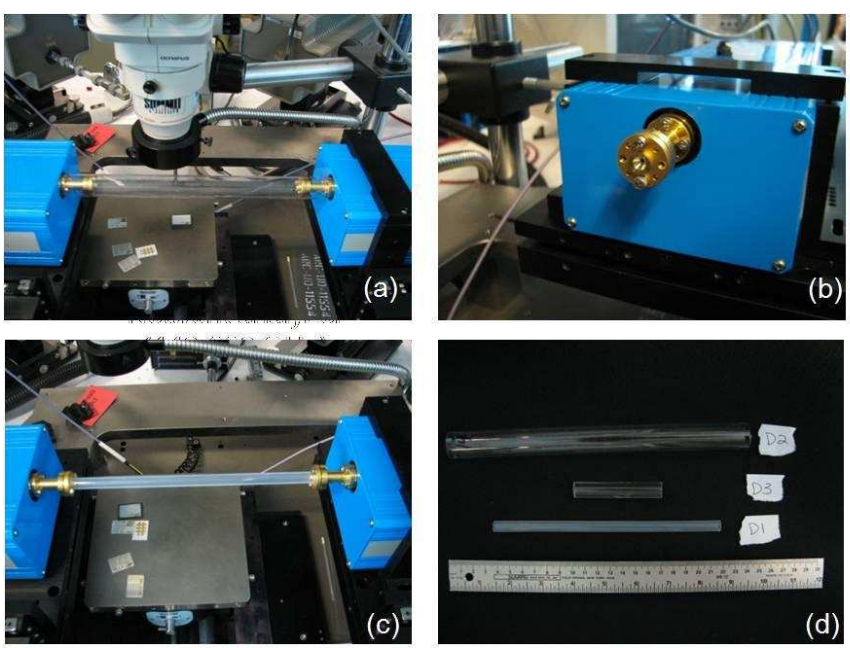

Figure A.3: Pictures show experimental set-up (at Cascade Microtech) with vector network analyzer (VNA). (a) Transmission measurements with tube D2 fitted between horns. (b) The horn attachment. (c) Transmission measurements with tube D1. (d) All tubes shown together.

$$
c_{l}\left(\theta_{l}\right)=\int_{0}^{2 \pi} F_{E}\left(\phi, \theta_{l}\right) d \phi,
$$

where $\theta_{l}$ is the launch angle for the $l^{\text {th }}$ mode, and is assumed to be $\cos ^{-1}\left(\frac{k_{z}^{l}}{n_{2} k_{0}}\right)$. An approximation for $S_{21}$ can be written as

$$
\frac{1}{c_{1}+c_{2}+\ldots c_{N}} \sum_{l=1}^{N} c_{l} e^{-j k_{z}^{l} d},
$$

where $k_{z}^{l}$ is the propagation constant for the $l^{t h}$ mode.

\section{A.2 Measurements}

As shown in Figure A.3, a vector network analyzer (VNA) was used to measure three hollow dielectric waveguides, labeled D1-D3 with two mechanisms of 

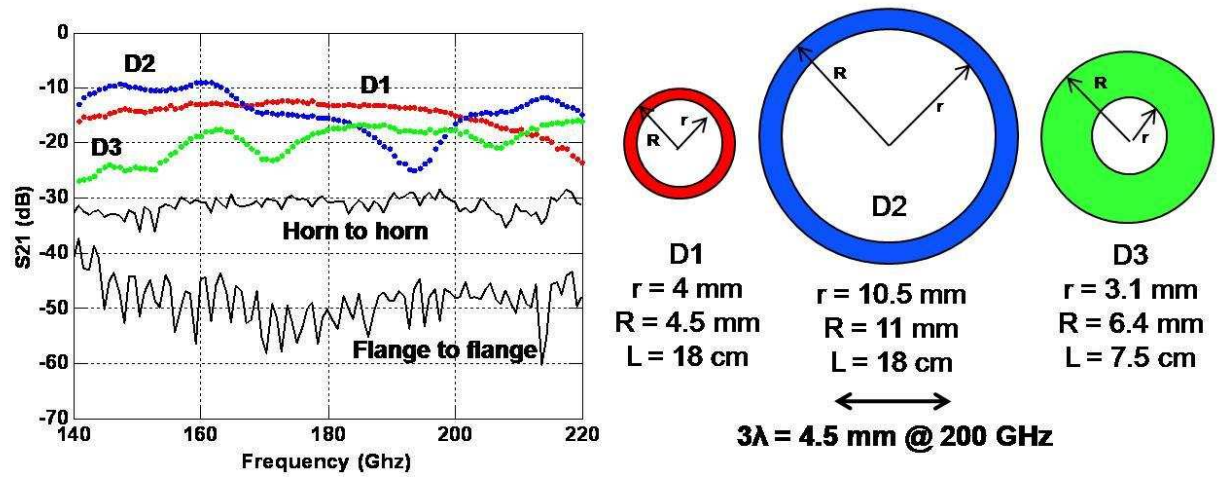

Figure A.4: (Left) Measured $S_{21}$ values for both flange and horn coupling mechanisms. Measurement for tubes D1, D2, and D3 are shown in red, blue and green, respectively. (Right) Each tube is drawn approximately to scale.

coupling energy into the tubes. Two-port S-parameters from 140-220 GHz were measured for five total configurations. The transmission measurement, $S_{21}$, across each configuration, is the parameter of interest and examined here.

The measurements of $S_{21}$ (plotted as power) are shown in left of Figure A.4 and were obtained as follows. First, WR-5 metal waveguide flanges were aligned $18.4 \mathrm{~cm}$ apart (WR-5 is designation meaning a hollow, rectangular waveguide with dimensions $1.29 \mathrm{~mm}$ x $0.65 \mathrm{~mm}$ ). Second, a metal, conical horn (Figure A.3(b)) was attached to each flange to increase the coupling between the WR-5 waveguide impedance and free space. The horn also serves to couple the rectangular waveguide of sub-wavelength dimensions to larger, cylindrical, tubes. Photos of the horn can be seen in Figure A.3. Finally, the three dielectric tubes shown in Figure A.3(d) were placed around each horn; the dimension of each tube can be seen in Figure A.4. Notice that these tubes are electrically large, several wavelengths in diameter, and not optimally designed. More refined waveguides will be discussed in last section. 
Several observations can be from the plot in Figure A.4. First, there is a gain of approximately $20 \mathrm{~dB}$ resulting from the directivity introduced with the horns. This occurs across the entire band. Secondly, the addition of the dielectric tubes increase gain by a further 10-20 dB (depending on the tube). Thus, there is a potential overall gain of nearly $40 \mathrm{~dB}$ relative to the unguided (free space) propagation. Given that most $\mathrm{THz}$ systems are limited by signal-to-noise ratio (SNR), this could provide critical capability.

Though SNR is important, we also must take into account dispersion. Tubes D2 and D3, having the largest diameter, clearly exhibit dispersive behavior. Small interference patterns can be seen across the band, which is to be expected with large waveguides supporting multiple modes. However, D1 displayed relatively non-dispersive behavior. In the next section, we compare the measurements to theoretically predicted values.

\section{A.3 Comparison of Model and Measurement}

Using (19), we can compute the expected value for $S_{21}$ using the complete propagation model. The plots in Figure A.5 show both the measured and theoretically computed values for $S_{21}$. Note that for theoretical calculations, we use $n=1.5-.001 \mathrm{j}$, which is a measured value for polyethylene, a common plas-

tic. [95]. The levels of the predicted values were adjusted to match the efficiency of the VNA. It can be seen that all three tubes support many hybrid modes, and therefore dispersion is expected because each mode will travel a different path length through the tube. The $S_{21}$ measurements for D2 and D3 appear to verify this hypothesis; there are 30 and 72 predicted propagating modes, respectively. 
While the model results agree qualitatively with the measurements, there is not exact agreement. We believe this is due to the strong sensitivity to the physical parameters, particularly permittivity and exact tube length. Slight changes in these values results in shifts in the null structure.
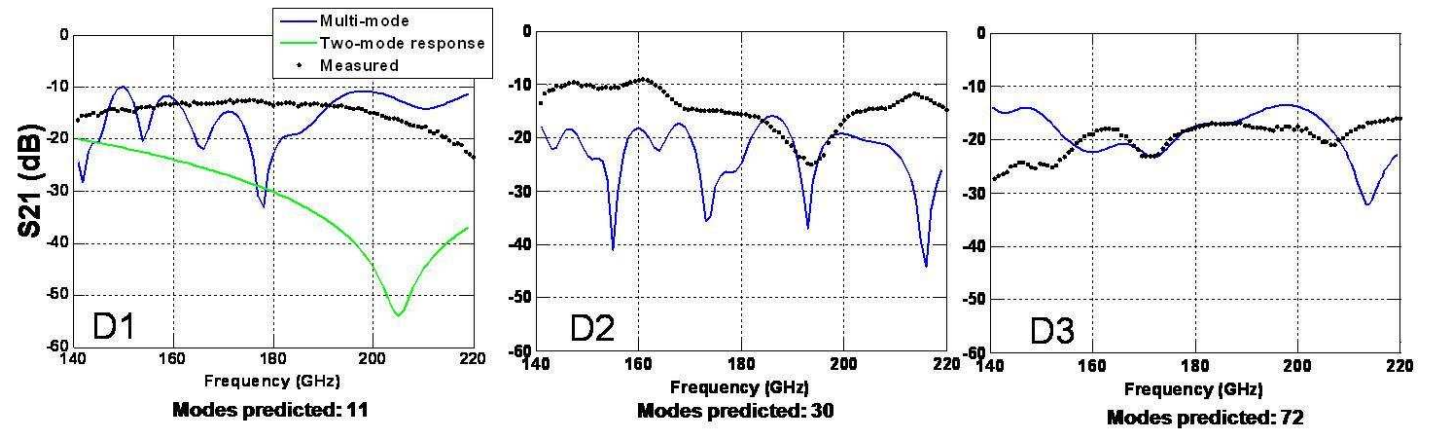

- - - Experiment Data _-Analytical model

Figure A.5: Comparison between $S_{21}$ measured and computed using propagation models. Included under each plot is the number of total modes supported.

However, the D1 tube clearly exhibits non-dispersive behavior indicating only one or two modes are actually propagating, as it has a flat spectral response with slight roll-offs at either end of the band. This is a qualitative disagreement from the computed values, which predict 11 propagating modes. Also plotted in Figure A.5(a), is the predicted response for D1 for only the two lowest order modes, which shows a distinct null at $205 \mathrm{GHz}$, and resembles the roll-off seen in the measurement (if the null occurred higher in frequency). It is possible that only the two lowest order modes remained at the end of the tube, therefore explaining the flat spectral content seen in the measurement. This non-dispersive behavior, combined with low attenuation, across an $18.4 \mathrm{~cm}$ span was a surprising result. Reasons for the proposed mode-stripping remain unknown; one possibility is surface roughness converting energy from one mode to another. Nonetheless, 
the method of launching RF energy into a hollow, dielectric tube is clearly an effective way of guiding terahertz radiation.

\section{A.4 Simulated Results}

As noted earlier, all of the measured waveguides are not optimal for transporting EM radiation in the terahertz regime. We now consider optimization of the waveguide using the propagation model. We will also shift the frequency range of interest to $0.4-0.9 \mathrm{THz}$, or $0.33-0.75 \mathrm{~mm}$ in wavelength. With proper dimensioning, single mode propagation is possible across a wide band because the so-called fundamental $H E M_{11}$ mode has no cut-off frequency, though does become loosely bound at low frequencies [96]. One important frequency-dependent parameter is power distribution. When placing multiple waveguides in close proximity, one must confine the power in the fundamental mode so that the signal does not interfere with neighboring components, and thus be tightly-bound. One can write the power distribution as the axial component of the Poynting vector. Using the field expressions from (8.1)-(8.3), we can write

$$
S_{z}(\rho, \phi)=\hat{z} \cdot\left(\bar{E} \times \bar{H}^{*}\right)
$$

where the radial field components can be obtained in a similar manor as in (8.1)(8.3). Power confinement can be quantified by taking the ratio of $S_{z}$ contained in the cross-sectional air core of the waveguide to the total. [97]

$$
P_{\text {ratio }}=\frac{\int_{A_{\text {core }}} S_{z}(\rho, \phi) d A}{\int_{A_{\text {total }}} S_{z}(\rho, \phi) d A}
$$

Looking at Figure A.6, a plot of power distribution for the $H E M_{11}$ mode, 

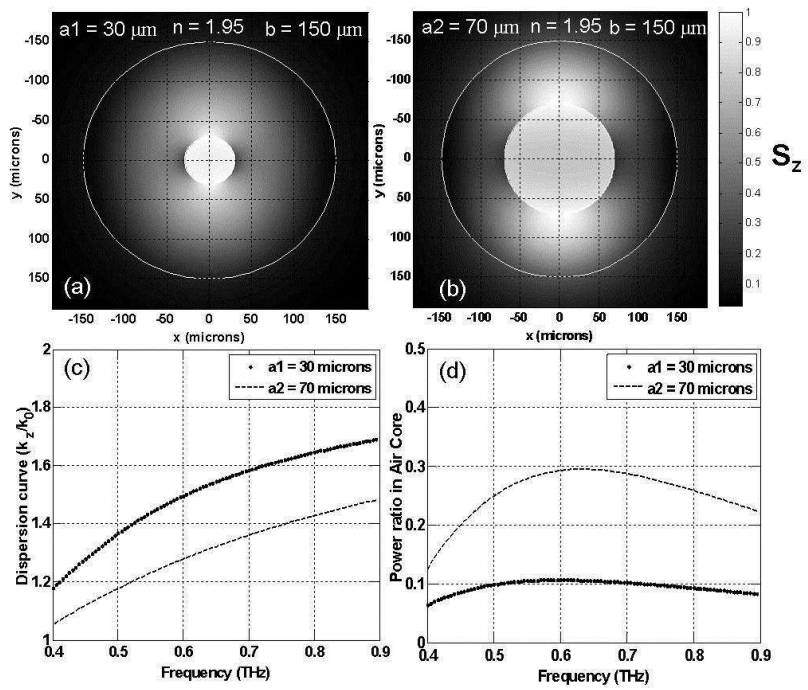

Figure A.6: Normalized axial power distributions for tubes with the same outer radius $(150 \mu \mathrm{m})$ but each with a different inner radius $(30 \mu \mathrm{m}$ and $70 \mu \mathrm{m}$, respectively) are shown in (a) and (b) for the fundamental mode. Dispersion curves $\left(k_{z} / k_{0}\right)$ and power ratios for each tube are shown in (c) and (d).

one can see that two waveguides with the same outer radius $(150 \mu \mathrm{m})$ and two different inner radii $(30 \mu \mathrm{m}$ and $70 \mu \mathrm{m})$ confine their respective power differently. The smaller air core actually contains higher power density, though the larger air core contains more total power because it has a larger area. It might initially be confusing that energy is traveling within the air void when the typical optical fiber carries most of its energy in the higher-indexed material. This can be explained by the constitutive relationship to Maxwell's equations: $D=\epsilon E$. The normal component of the D-flux must be constant across the boundary. Therefore, a vertically polarized mode, provides a higher E-field in the lower indexed side of the boundary (air core) [98]. If the inside diameter small enough, a high percentage the total power can be confined inside the air core. However, the inside diameter is too small, then there isn't enough area to carry a sufficient percentage. 
Power confinement is dependent, of course, on frequency. In Figure A.6(c),(d), we show both power ratio and dispersion curves for the aforementioned tubes. It is clear that one has a higher power confinement than the other, and in general an optimal point can be found over a range of frequencies. The dispersion curve is also flattened for the larger core, which helps minimize signal broadening.

Clearly there are trade-offs to consider when choosing waveguides dimensions and materials, and these choices will affect the power confinement. Ideally, the dispersion curve is flat, power is ideally confined completely, and attenuation is zero. However, it is not possible to choose parameters to realize optimal performance in every domain, and the parameter choice will be dictated by the specific applications. 


\section{Appendix B}

\section{Generation of Receiver Operating Characteristics}

The receiver operating characteristic (ROC) curve is considered one of the standard ways to analyze the performance of a detection algorithm, as it shows the complete detection performance. ROC curves are a plot of probability of detection, $P_{d}$, against probability of false alarm (or false positive), $P_{f a}$ and are often used in a variety of fields such as radar and biomedical diagnosis. It can be easy to achieve $100 \%$ chance of detection, so long as there is also $100 \%$ chance of false alarm. In addition, one can also manipulate detection performance by selecting beneficial false alarm rates. Indeed, some detection algorithms perform better at very low false alarm rates than at relatively high ones.

Figure B.1(a) shows 500 realizations of two populations, both generated as Gaussian random numbers with identical variances. The first population has a mean of 0.5 , while the second population has a mean of 0. Figure B.1(b) shows the probability density functions for each population. In order to make a decision, a threshold is drawn in between the distributions, without knowing which distribution the data actually comes from. The probability of detection is calculated according to (4.11), which corresponds to all average number realizations from population 1 above the threshold. Similarly, the probability of false alarm is cal-

culated using the same threshold using (4.12). Both of these numbers, $\left(P_{f a}, P_{d}\right)$, corresponding to one point on an ROC curve. The threshold is then swept across a range of values, and with each move, a different point on the ROC curve is 
calculated.

Figure B.2(a) shows several different ROC curves. The first ROC curve is a ideal, with $P_{d}=1$, while $P_{f a}=0$. The second curve shows a very good ROC curve. For example, this curve contains the point with $P_{d}=0.95$ and $P_{f a}=.08$. The third curve shows a useless ROC curve that is the same as flipping a coin to decide whether the data comes from the first or second population. That is, there is a $50 \%$ chance of detection with $50 \%$ chance of false alarm. ROC curves are used throughout this dissertation to compare the performance of multiple detection methods. 


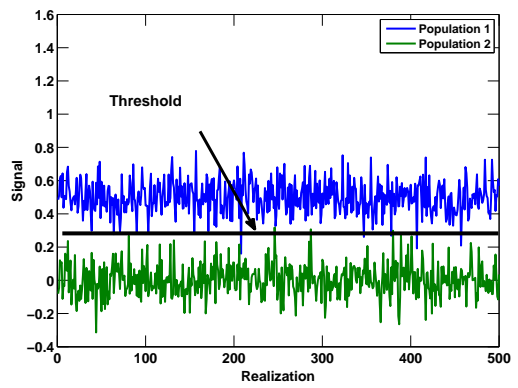

(a)

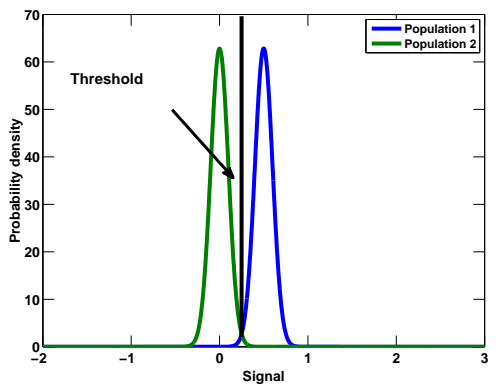

(b)

Figure B.1: (a) Sequence of random numbers drawn from a Gaussian distribution with mean of 0.5 (blue) and 0 (green). (b) Probability density distribution corresponding to each distribution.

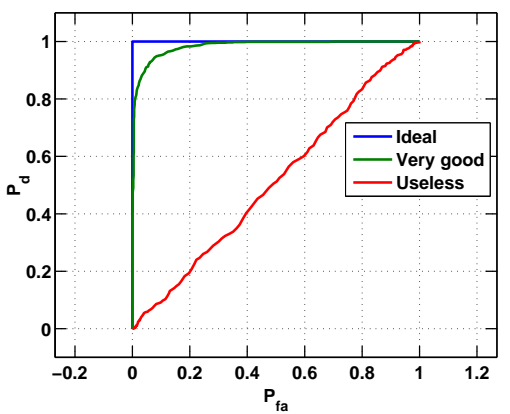

(a)

Figure B.2: (a) Three ROC curves shown: ideal curve (blue), very strong curve (green), and a useless curve (red). 


\section{Appendix C}

\section{Computationally Efficient Implementation of Synthetic-Aperture Imaging}

This section outlines the $\mathrm{THz}$ SA broadband implementation that was described in Chapter 5. Equations (5.4) and (5.7) describe an imaging algorithm that a frequency-domain adaptation of a 2-D phased-array beamformer. Typically phased-array beamformers form plane waves that are focused to infinity; and the formulation shown here is near-field beamforming in which the array focuses to a point relatively close to the array. While this technique allows for 3-D imaging, there are computational challenges to doing this.

Equation (5.4) requires the total number of operations to be $\mathrm{M} \times \mathrm{N}$ by the total numbers of voxels in the desired image. Suppose a $200 \times 200$ synthetic array of elements (with 100 microns spacing) is used to image a 3-D volume that consists of $200 \times 200 \times 100$ voxels. Implementing (5.4) directly results in 160 billion operations, which is before broadband averaging in (5.7). Because the operations involve complex numbers, the actual amount of computation is more. In addition, the computational overhead of using loops in a programming language such as MATLAB makes doing this type of calculation increasingly difficult. One option that could mitigate this problem is by performing multiple operations simultaneously, or vectorizing. However, this would entail creating very large multi-dimensional matrices which would exceed the amount of available memory on most machines. 
The approach used in this work utilizes the computation efficiency of the 2-D Fourier transform. Instead of multiplying all elements in the 2-D array by an appropriate phase, a convolution is used. A phase kernel is first constructed that focuses directly down toward the center of the array.

This approach may appear to do additional unused operations, and does contain some extra complexity. However, it is significantly faster than the alternative. Equation (5.4) can be represented as a convolution integral, where the received electric field is convolved in two-dimensions with a complex tapered beamforming kernel. That is,

$$
C_{N B}\left(f_{q}, \vec{r}_{h j l}\right)=E\left(x_{m}, y_{n}, f_{q}\right) \star\left[H_{m n}\left(\vec{r}_{h j l}, f_{q}\right)\right],
$$

where the notation $\star$ represents a 2 -D convolution. The $\mathrm{M} \times \mathrm{N}$ matrix, $H_{m n}\left(\vec{r}_{h j l}\right)$, includes phase adjustments at all sensor positions for a desired imaging pixel directly below the center of the array. Only one depth image is achieved per convolution, therefore this process must be repeated across all depth slices to create the 3-D image. The process can be visualized in one-dimension in Figure C.1.

For each pixel, each of the received signals are multiplied by the appropriate phases/amplitudes and added together coherently. Each pixel in the 2-D image is created by the delay that occurs in each dimension of the convolution. The convolution does produce unwanted pixels in the resulting convolution, which are shown in red in Figure C.1. These edge effects are truncated from the final images. The Gaussian taper included in $H_{m n}\left(\vec{r}_{h j l}\right)$ forces the resulting convolution of all perfectly constructive elements in the convolution to unity. By utilizing the 2-D Fourier transform, a 2-D convolution significantly reduces the number of 


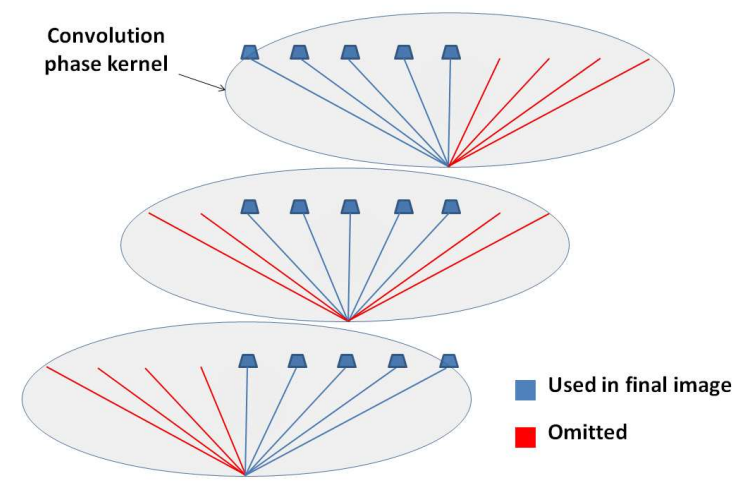

Figure C.1: One-dimensional Synthetic array implemented by convolving the raw complex image data with complex phase kernel corresponding to appropriate ray paths. The middle of the resulting operation is cropped out to create the final image

computations needed in (5.4) to produce an image. 


\section{Appendix D}

\section{Characterization of Virtual Source}

In order to gain intuition for how the virtual source operates as a diverging beam, it is important to properly characterize the virtual source. This is done to ensure that the beam is in fact diverging below the physical focal point of the one-inch lens, and to determine to what degree it is diverging. A knife-edge test was conducted at several points around and below the focal point of the one-inch polyethylene focusing lens. Essentially, this is an additional method of measuring the emitted beam pattern at different $\mathrm{THz}$ frequencies. Using this information, the cone angle of the diverging beam can be calculated.

Figure ?? shows the configuration of the knife edge test. An aluminum plate with a sharp edge was scanned across the beam at the focal point of the lens. This was repeated again as the plate was moved further and further below focal point. The knife edge test occurred at varying depths between 0 and $8 \mathrm{~mm}$ below

the focal point of the lens. This process was done in both the E-plane and the H-plane of the photoconductive antenna.

Figure ?? shows the result of the knife edge test at $0.6 \mathrm{THz}$ after taking the discrete derivative across position, either $x$ or $y$, for the E-plane and H-plane profiles, respectively. Figure D.2(a) shows the beam profile along the E-plane, while Figure D.2(b) shows the beam profile along the H-plane. Each of the two profiles looks qualitatively similar. Both start at a point and widen as $z_{0}$, the depth below the virtual source, increases. The beam angles are also quite similar. 


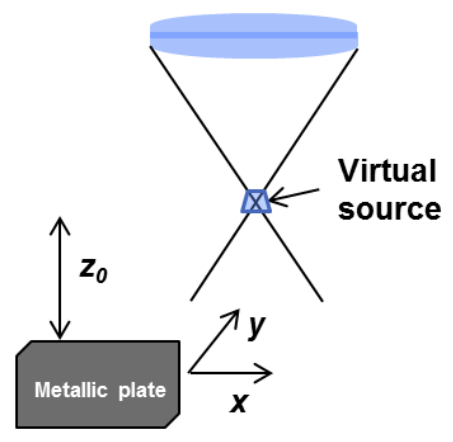

(a)

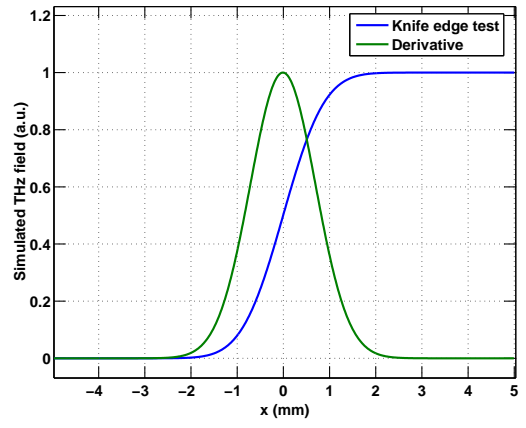

(b)

Figure D.1: (a) Demonstration of knife edge test on $\mathrm{THz}$ virtual source using a metallic object with a fine edge that is swept across the $\mathrm{THz}$ beam. (b) Simulated knife edge test data, and corresponding beam profile found by taking the derivative across position.

The E-plane profile spreads at $22^{\circ}$ (full-angle), compared to $25^{\circ}$ for the H-plane. 


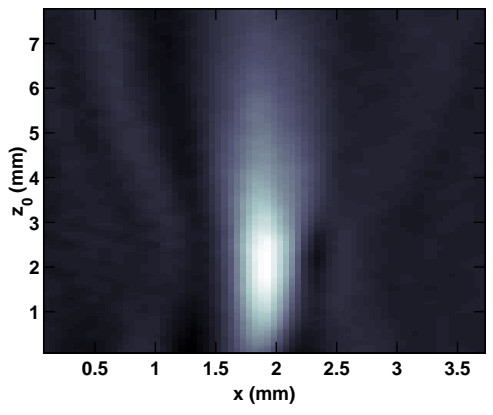

(a)

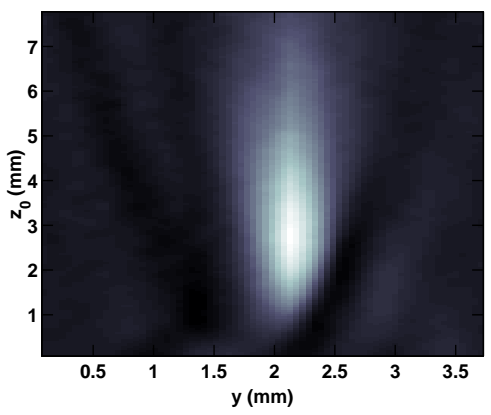

(b)

Figure D.2: (a) Beam pattern at $0.6 \mathrm{THz}$ along the E-plane of the antenna below focal point, or virtual source. (b) Beam pattern at $0.6 \mathrm{THz}$ along the H-plane of the antenna below focal point, or virtual source. 


\section{Appendix E}

\section{Virtual Source Drift Correction}

One important item that was observed during the implementation of the SA imaging algorithm was that the physical focal point of the one-inch lens shifts as a function of frequency. This effect can be measured via computing (5.2) and imaging a small target such as a ball bearing.

There are two main reasons for this shift. The first involves the shift of the physical focal point of the one-inch lens. This is likely due to the polyethylene focusing lens being closer to the near field of the transmitting dipole antenna, for higher frequencies. Essentially, the transmitted beam becomes a collimated beam, creating the focal point relatively close to the lens. Conversely, lower in frequency, the transmitted radiation more closely resembles a diverging beam, lengthening the focal point. Unfortunately, the exact calculation of the far field distance isn't possible as the antenna structure is proprietary.

This effect was verified with a knife edge test, similar to the one conducted in Appendix D. An aluminum plate was scanned across the beam at various depths above and below the focal point of the lens. The derivative with respect to horizontal position (of the knife) was used to extract the beam profile as a function of both depth and frequency. At each frequency, the location of the focal point was achieved by searching for the depth corresponding to the smallest beam waist. From this, it can be empirically found how much the focal point drifts as frequency increases. 


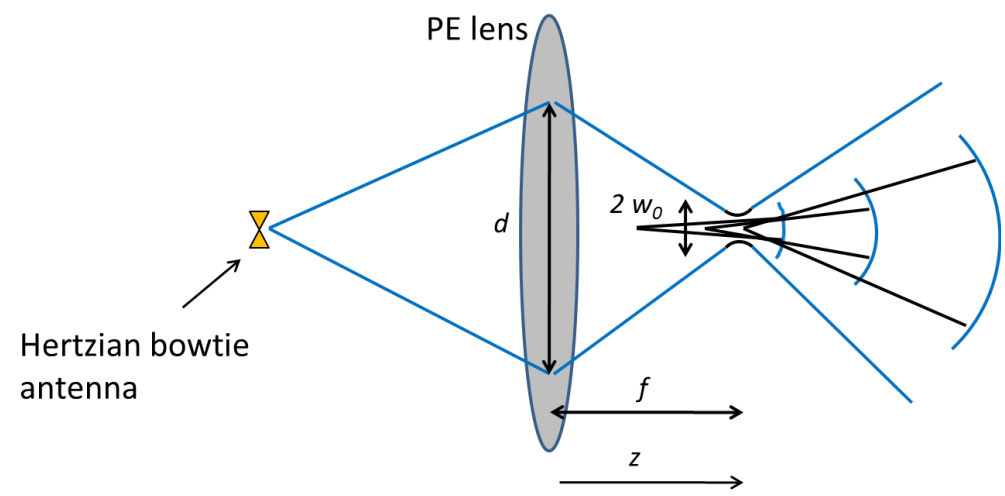

Figure E.1: Diagram of a Gaussian beam showing that the radius of curvature can vary when close to the beam waist

The second reason that the virtual source shifts can be explained by a simple model for a Gaussian beam. The lens focuses THz energy to a focal volume; and below this volume the beam diverges, as shown in Figure E.1. A Gaussian beam model predicts that in the focal volume, the radiation propagates forward as a plane wave that is tapered radially by a Gaussian profile, which equates to an infinite radius of curvature of the diverging beam. Therefore, the radius of curvature does not linearly grow with the depth below the focal point. The electric field for a Gaussian beam is given as

$$
E(\lambda, r, z)=E_{0} \frac{w_{0}}{w(z)} e^{\frac{-r^{2}}{w^{2}(z)}} e^{-i k\left(z+\frac{r^{2}}{2 R(z)}\right)}
$$

where $\mathrm{z}$ is along the axial dimension of the Gaussian beam. The effective radius of curvature, $R$, of the beam can be written as

$$
R(z, \lambda)=z\left[1+\left(\frac{1.22^{2} \pi^{2} f_{\text {num }} \lambda}{z}\right)^{2}\right]
$$

where $f_{\text {num }}$ is the f-number of the lens. Therefore, the radius of curvature becomes 
greater as function of frequency.

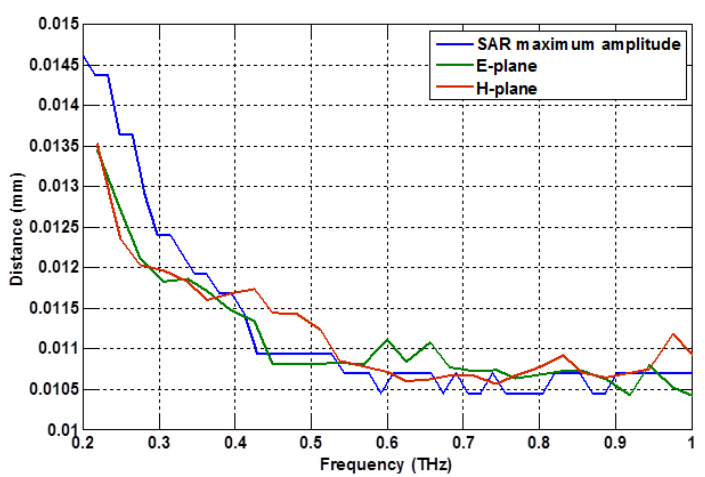

Figure E.2: Virtual source drifting as a function of frequency. E-plane and Hplane curves are generated using measured focal points and predicting radius of curvature using Gaussian beam theory. SAR maximum amplitude was obtained from 3-D ball bearing image to find location of virtual source.

The effect of the beam waist (focal point) drifting and the radius of curvature increase are distinct events; and both have an impact on virtual source location. Although one of these effects (focal point) was determined empirically by a knife edge test, both drifting mechanisms can be combined to estimate the effective virtual source height, which is plotted below in Figure E.2 for E-plane (green) and H-plane (red). For comparison, a small ball bearing $1 \mathrm{~mm}$ in radius was imaged in a SA configuration (roughly $1 \mathrm{~cm}$ below the broadband focal point location). At each frequency, a depth profile of the ball bearing is created. The maximum of each depth profile is plotted in Figure E.2, and provides an independent measure of effective virtual source location, relative to the lens.

Considering that the virtual source drifts away toward the lens inversely proportional to frequency, it makes sense that it drifts linearly away from the source as a function of wavelength, $\lambda$. To mitigate the issue of a moving virtual source, a simple linear model is constructed of the virtual source position as a function 
of frequency. The estimated radius of curvature, $\hat{R}(z)$, can be written as

$$
\hat{R}(z)=R_{z 0}+k \lambda
$$

where $k$ is the fitting factor, and $R_{z 0}$ is the radius with wavelength equal to zero. The linear fit and the maximum of each SA depth profile are plotted as a function of wavelength in Figure E.3.

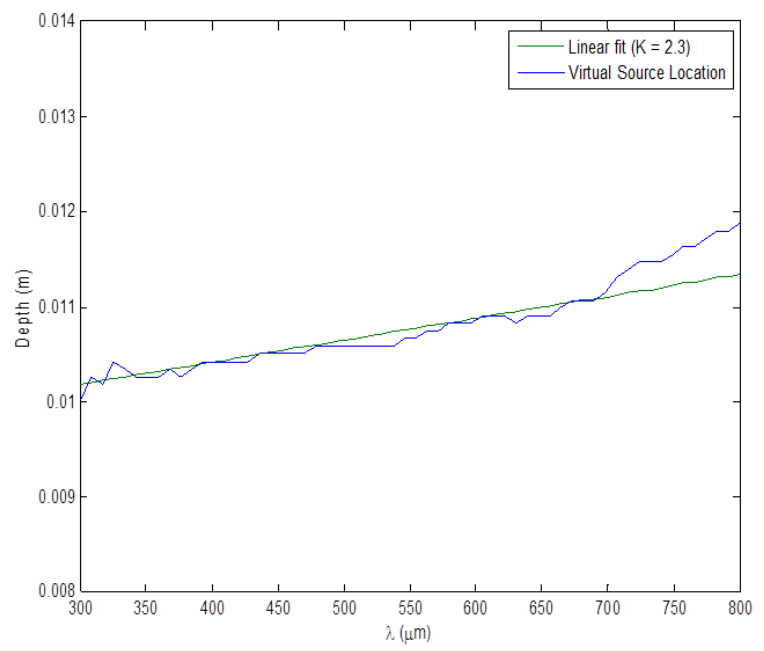

Figure E.3: Use a linear fit to address virtual source drift as a function of wavelength.

Figure E.4(a) below shows an image plotting the real portion of the narrowband focused field directly over the ball bearing as a function of both focusing depth and frequency as computed from (5.4). This striation pattern is reasonable showing strong constructive interference at the true focal depth of $1 \mathrm{~cm}$. However, one can notice the amplitude modulation that drifts as a function frequency.

Using (E.3) to correct for this, a new striation pattern can be computed, shown in Figure E.4(b). The new striation pattern shows the amplitude modulating the 


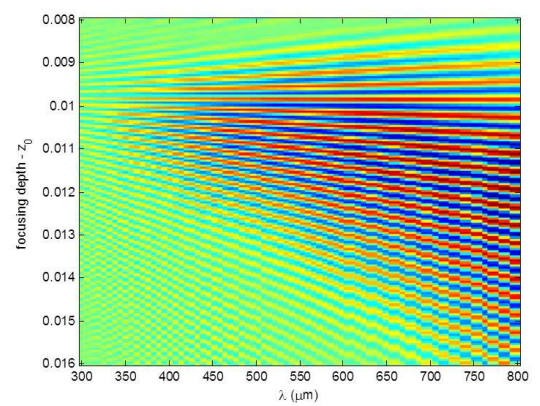

(a)

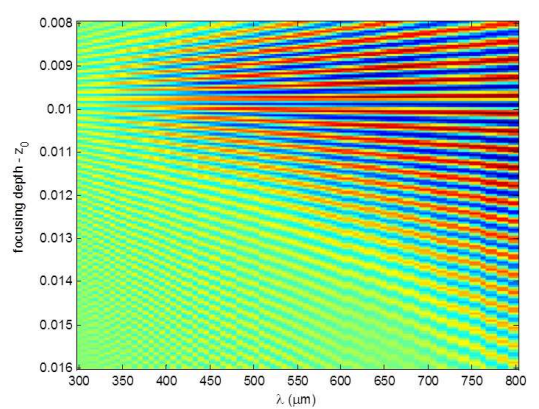

(b)

Figure E.4: (a) Uncorrected striation pattern plotting SA focused field as function of both focusing depth and wavelength. The field oscillates between negative and positive values across depth and frequency. However, the field is in-phase across frequency only at one depth. (b) Corrected version of striation pattern using (E.3).

real portion of the field to be leveled out. In effect, this increases efficiency because the average real portion of the field is higher after correction. After broadband averaging from (5.7), the peak corresponding to the $1 \mathrm{~cm}$ will be higher, with the sidelobes and local minima occurring roughly the same as without the correction.

Figure E.5 shows the narrowband depth profile corresponding to a SA image of a small ball bearing at $0.3 \mathrm{THz}$ (in blue). Also shown in Figure E.5 is depth profile with and without the correction factor described in (E.3) with a frequency average of $0.3-0.5 \mathrm{THz}$ (shown in green and red, respectively). Clearly the correction is improving the efficiency with these bandwidths. However, for most practical bandwidths, such as those used to produce images in Section 5.4, this correction factor is negligible. 


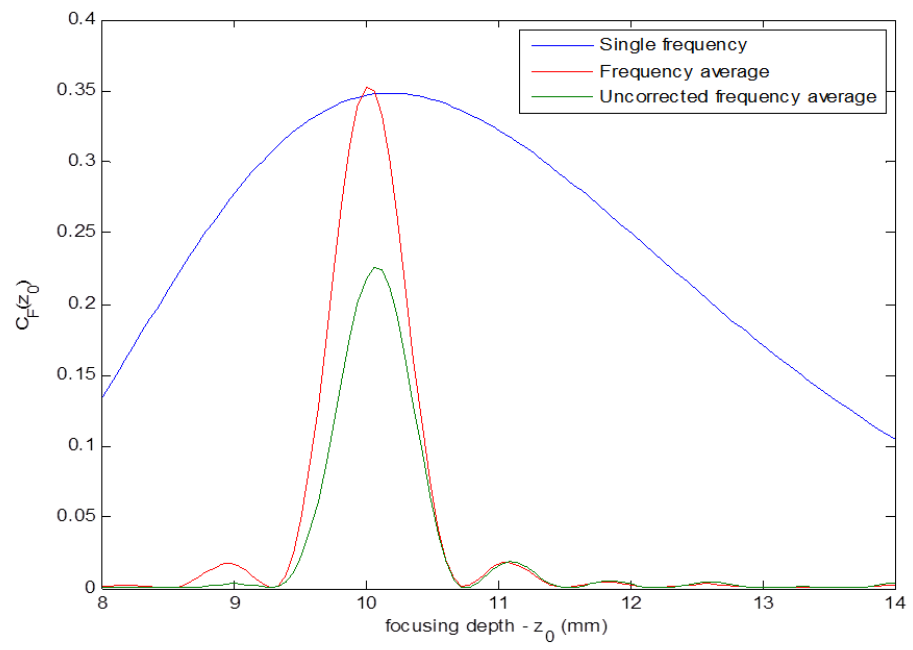

Figure E.5: Depth profile of corresponding to the center of a single frequency SA image of a small ball bearing shown in blue. Uncorrected and corrected depth profiles are shown in green and red, respectively. 


\section{Appendix F}

\section{Synthetic-Aperture Refraction Correction for Dielectric Structures}

All SA imaging formulations have been presented assuming the propagation paths were contained in free space. However, results in Section 5.3 and 5.4 used an alternative method to take account refraction of a planar dielectric/air interface. Using geometric optics, a $\mathrm{THz}$ ray first propagates from the sensor position to the surface and then penetrates (while refracting) into the dielectric filler. There are two different ray paths involved in this situation, as shown in Figure F.1. The comprehensive optical path is altered after accounting for refraction.

Snells Law relates the incident angle of a ray from material with permittivity, $\epsilon_{0}$, into a material with filler permittivity, $\epsilon_{f}$, which can be written as

$$
\sqrt{\epsilon_{0}} \sin \left(\theta_{1}\right)=\sqrt{\epsilon_{f}} \sin \left(\theta_{2}\right)
$$

or

$$
\sqrt{\epsilon_{0}} \frac{\sqrt{x_{1}^{2}+y_{1}^{2}}}{d_{1}}=\sqrt{\epsilon_{f}} \frac{\sqrt{x_{2}^{2}+y_{2}^{2}}}{d_{2}},
$$

where $\theta_{1}$ is the incident ray and $\theta_{2}$ is the refracted ray. Both are measured with respect to the filler materials' surface normal. A cross sectional view of both incident and refracted rays can be seen in Figure F.1. Using the paraxial approximation which assumes $\theta$ is relatively small (generally less than 15 degrees), (F.2) becomes 


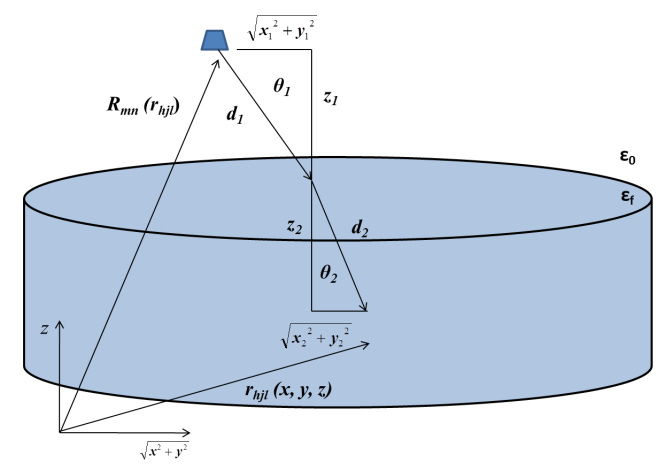

Figure F.1: Cross-sectional view of a light ray entering dielectric filler material, with relative permittivity, $\epsilon_{f}$, and scattering off an particle within filler material.

$$
\sqrt{\epsilon_{0}} \frac{\sqrt{x_{1}^{2}+y_{1}^{2}}}{z_{1}}=\sqrt{\epsilon_{f}} \frac{\sqrt{x_{2}^{2}+y_{2}^{2}}}{z_{2}}
$$

Recall that $\sqrt{x_{1}^{2}+y_{1}^{2}}$ and $\sqrt{x_{2}^{2}+y_{2}^{2}}$ have to add to the horizontal components of $\vec{R}_{m n}$ subtracted from $\vec{r}_{h j l}$ in (5.4). This can be written as

$$
\left|\vec{R}_{m n}(x, y, 0)-\vec{r}_{h j l}(x, y, 0)\right|=\sqrt{x_{1}^{2}+y_{1}^{2}}+\sqrt{x_{2}^{2}+y_{2}^{2}} .
$$

Substituting into (F.3), the horizontal distance the ray travels free space above the filler material, $\sqrt{x_{1}^{2}+y_{1}^{2}}$, can be written as

$$
\sqrt{x_{1}^{2}+y_{1}^{2}}=\frac{\left|\vec{R}_{m n}(x, y, 0)-\vec{r}_{h l}(x, y, 0)\right|}{\left(1+\sqrt{\frac{\epsilon_{0}}{\epsilon_{f}}} \frac{z_{2}}{z_{1}}\right)}
$$

Therefore, the total path length for the ray above the interface can be written as

$$
d_{1}=\sqrt{\left(x_{1}^{2}+y_{1}^{2}\right)+z_{1}^{2}} .
$$

And the path length inside the filler material, $d_{2}$, can be written similarly. 
The total effective optical path length can be written as $d_{1}+\sqrt{\epsilon_{f}} d_{2}$. Now, these two path lengths can be combined with different phase velocities into a new SA imaging formulation. The modified SA imaging kernel can be described by

$$
e^{i 2 k_{0}\left[d_{1}+\sqrt{\epsilon_{f}} d_{2}\right]}
$$

where $d_{1}$ and $d_{2}$ depend the refractive index of the filler medium and each combination of sensor position and imaging pixel. 